Disttribution Caltegony:

Mathematics and computerers $((\mathrm{U} C-32)$

$\mid A N L-777-811$

AARGONNE WATIIONAL LLABORAATORY

9700 South Cass Aavenure

Argoonne, II Inimoits 60439

AA SURVEY COF WUIMERICALL METHODS

FOR HNORAULIIC TRRANSIIENTS

by

G. KK. Leaff

Applifed Mathemattics Diviision

ant

T.. C. Chawlia

Reactor Anallysiis and Safiety Divivision

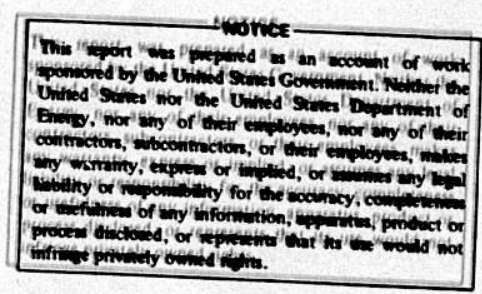

Octodber 79977 

TABLLE COF CONTENTS

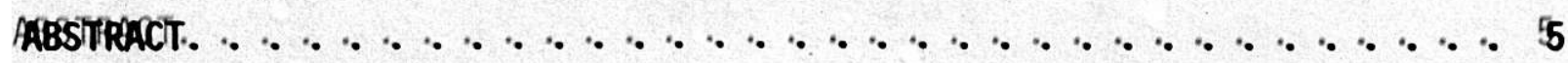

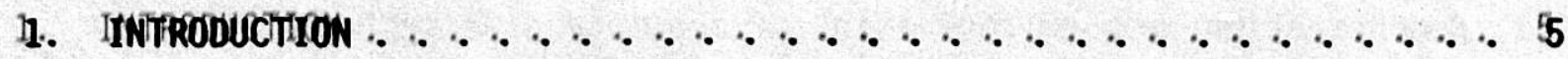

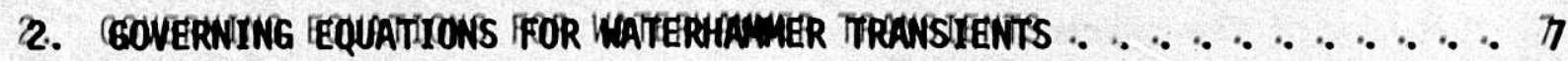

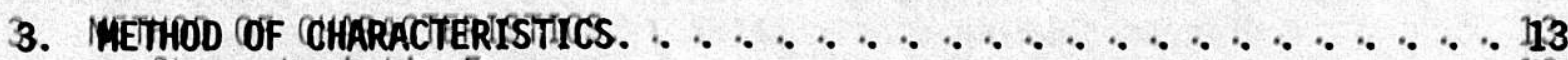

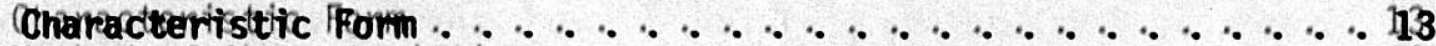

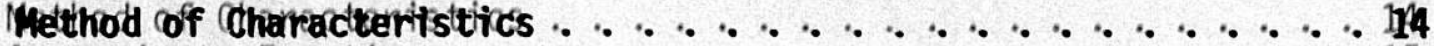

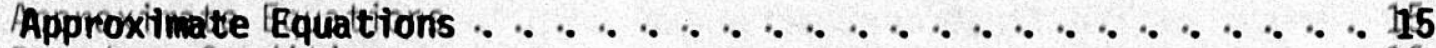

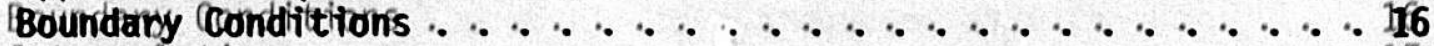

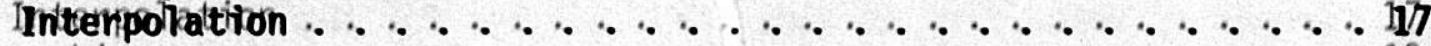

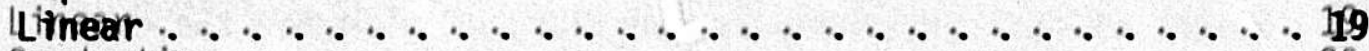

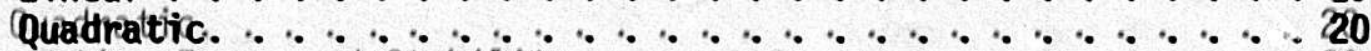

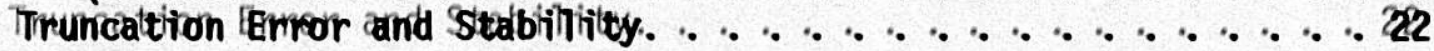

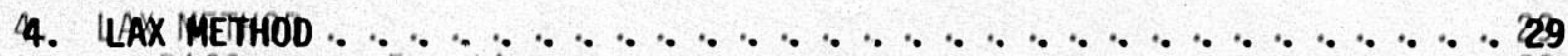

Difference Equations. . . . . . . . . . . . . . . .

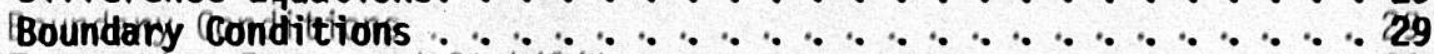

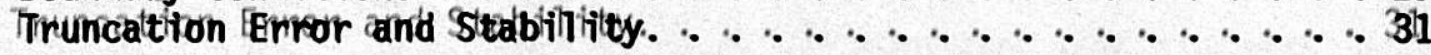

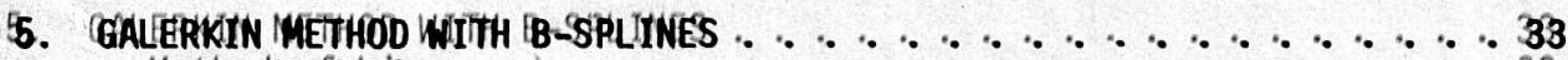

Method of lithes $\ldots \ldots \ldots \ldots \ldots$

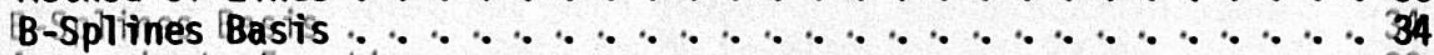

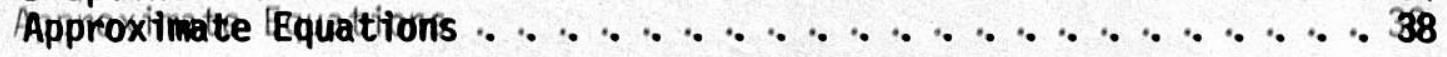

6. COMPAARIISON COF LIINEARR AND QUUAOPRATIIC IINTERRPOLATIION

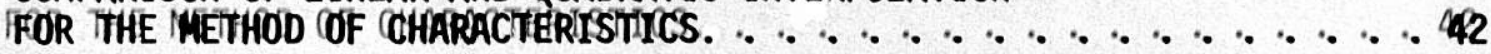

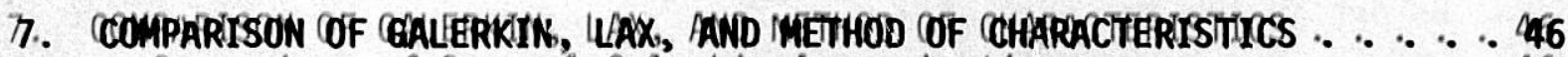

comparison of Several Galention Approximattionis ..........46

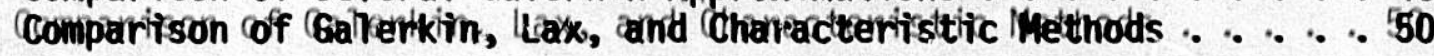

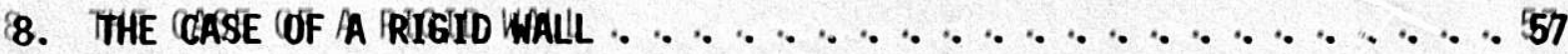

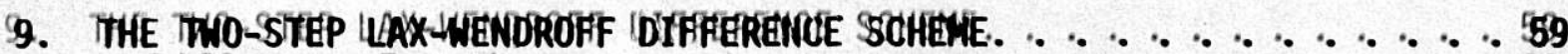

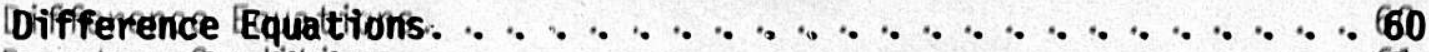

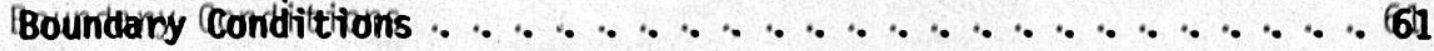

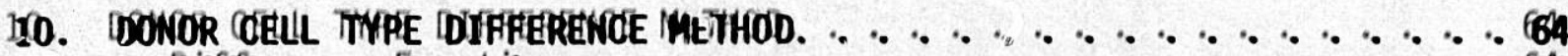

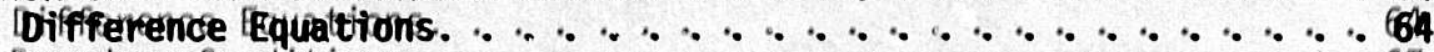

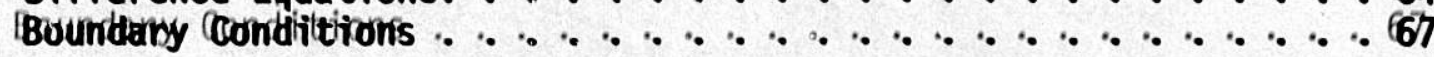

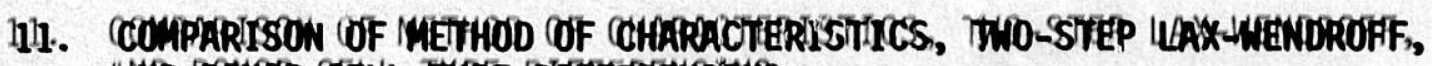

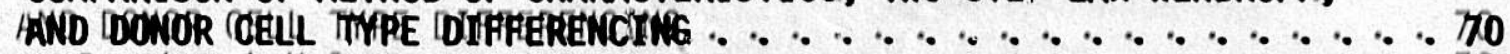

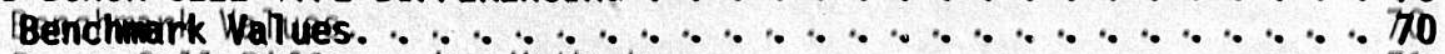

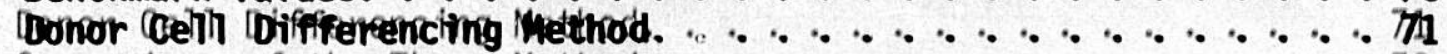

comparisson of the three Methrodts .......................

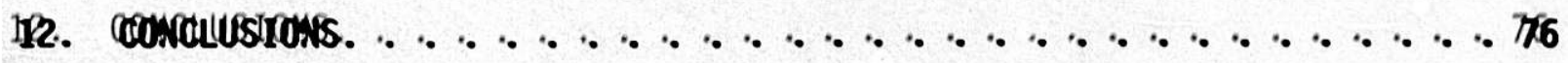

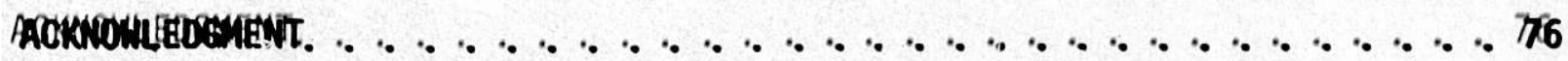

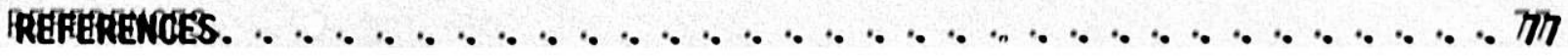




\section{LLIST OOF PFIRURES}

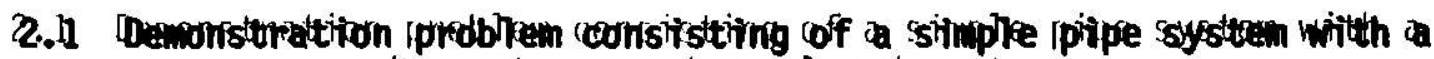

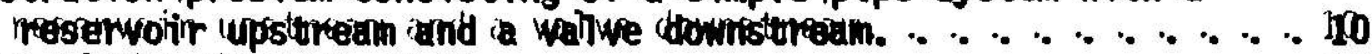

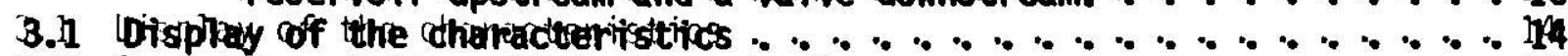

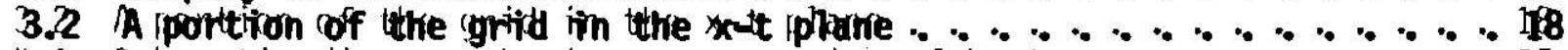

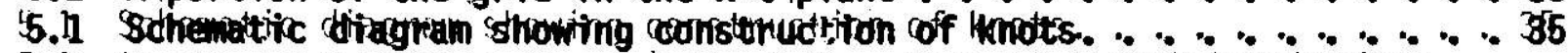

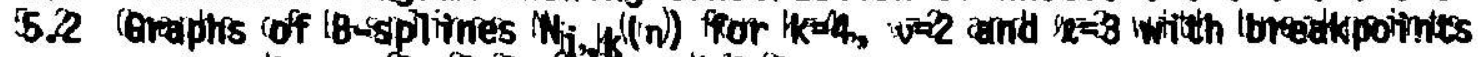
dit $n=0,0.3,0.7)$ ant $1.0 \ldots \ldots \ldots$

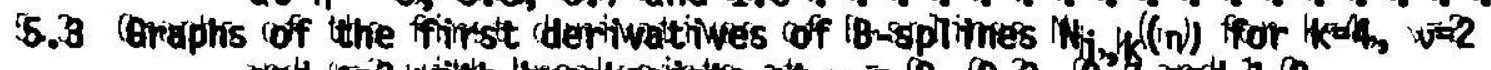

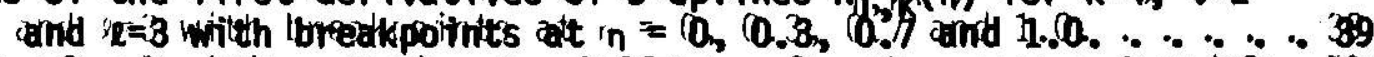

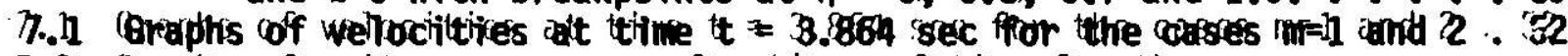

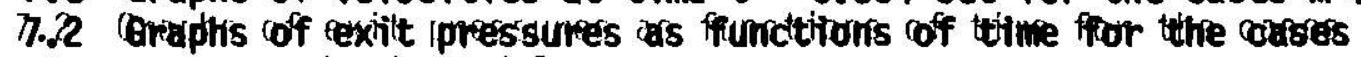

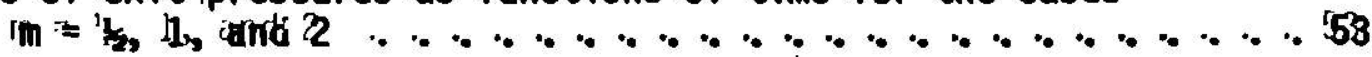

\section{LLISST COF TPABLLES}

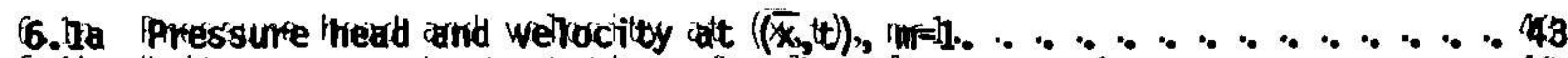

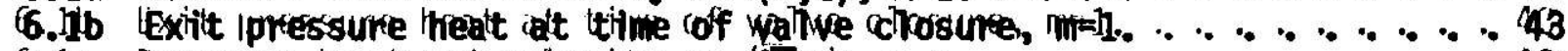

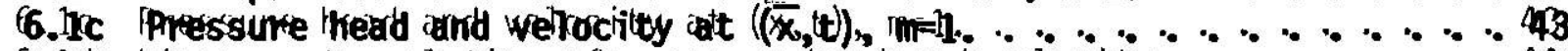

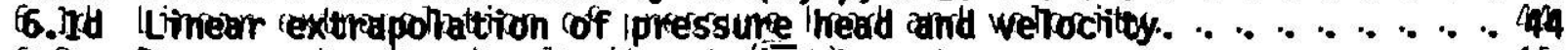

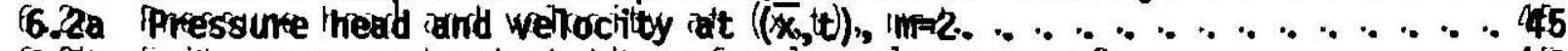

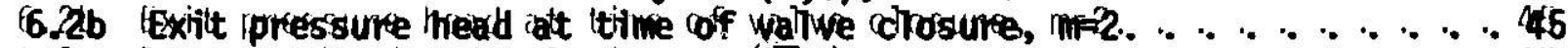

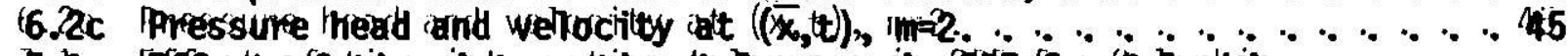

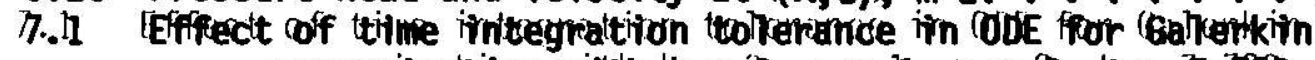
approximattion witth $k=3, v=11,2=22, t=7.7728 \ldots . \cdots 47$

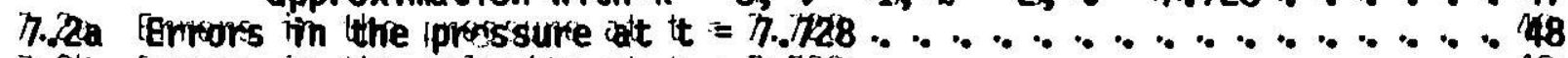

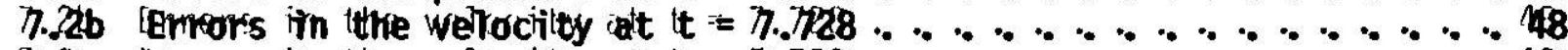

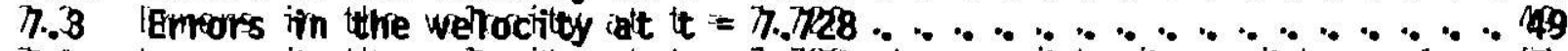

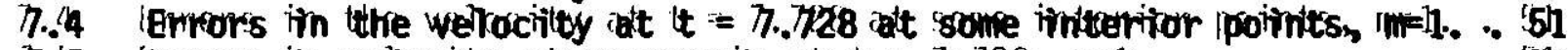

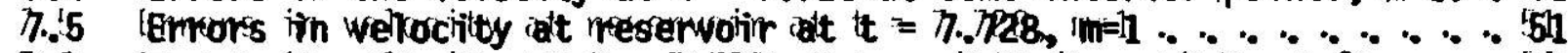

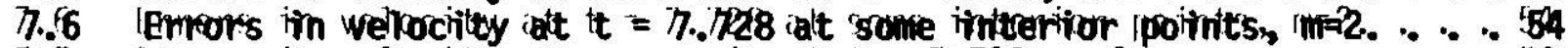

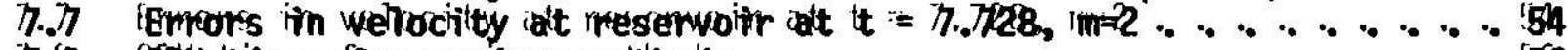

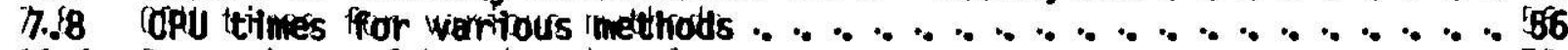

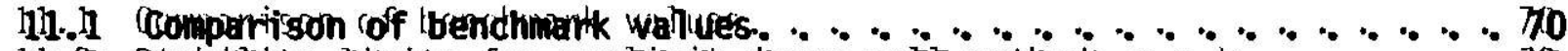

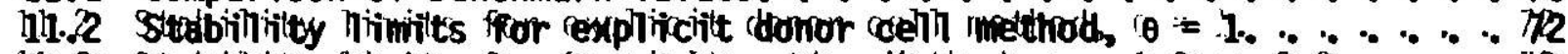

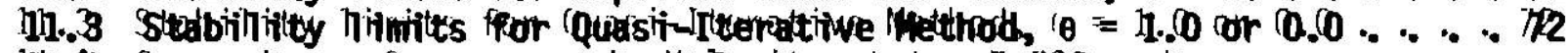

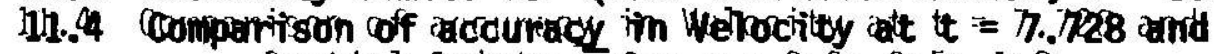

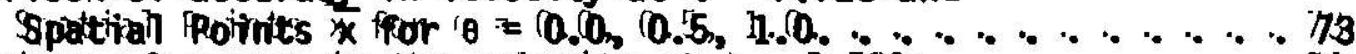

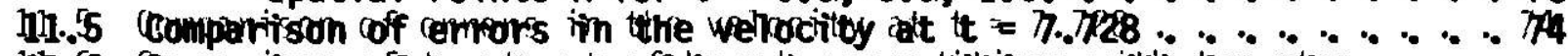

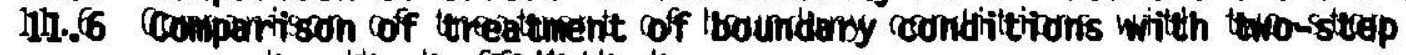

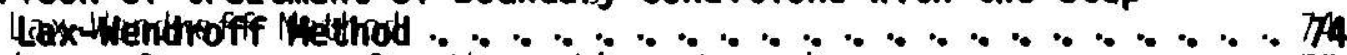

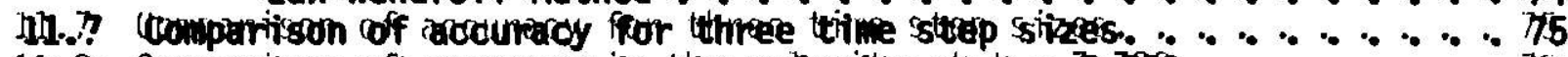

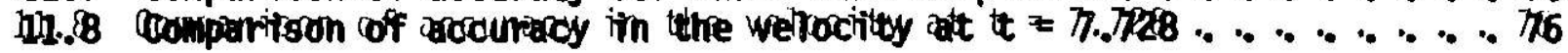


AA SURVEY OF NUMERITCAL METHCODS

FOR HMORAULIC TRRANSIIENTIS

toy

G. K. Leaff and T. (C. Chawita

\begin{abstract}
AABSTIPACT

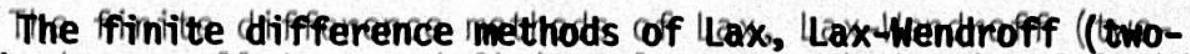

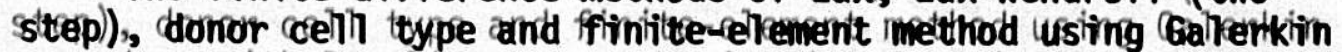

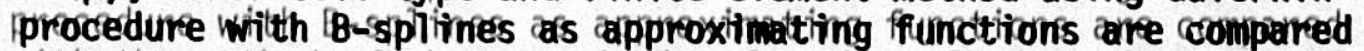
with the metthod off characteriisttics for the ssolutition of walter-

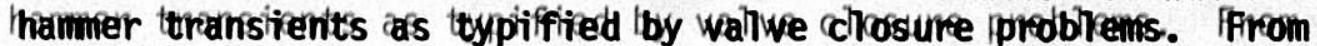

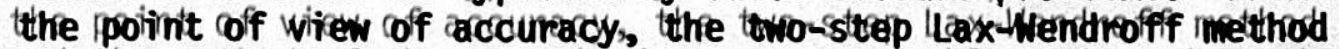

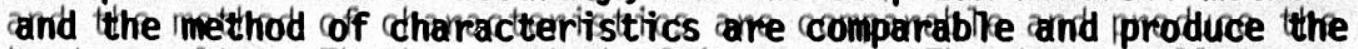

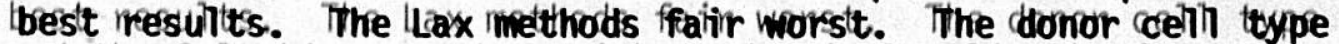

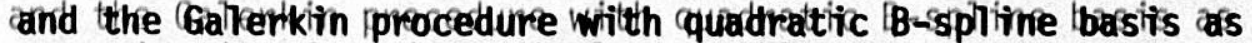

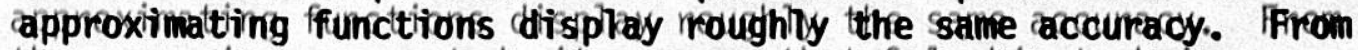
the compariison ipresented, iitt applears thatt Galkenkion trechnirque

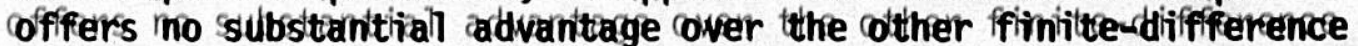

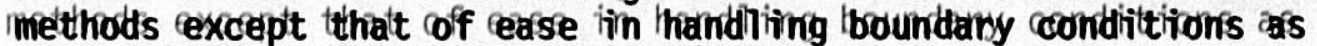
compared to firnitte-diffiference methodis.
\end{abstract}

\title{
11. IINTRODUCTIION
}

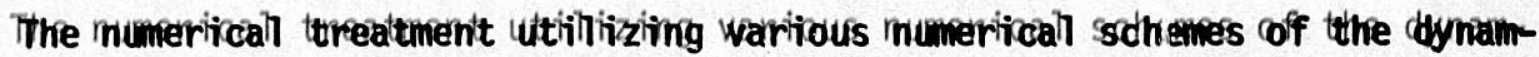

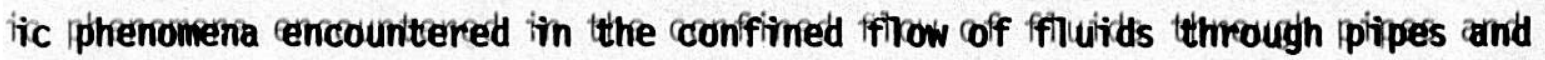

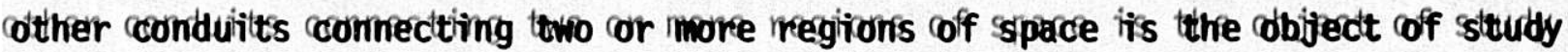

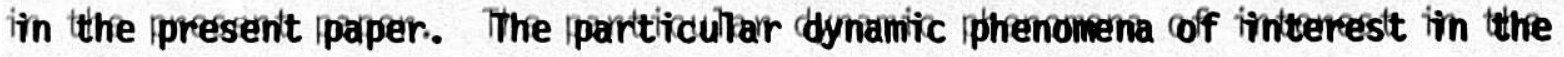

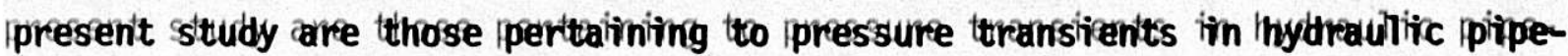

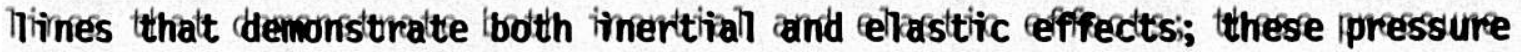

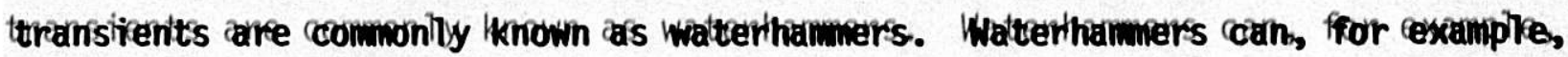

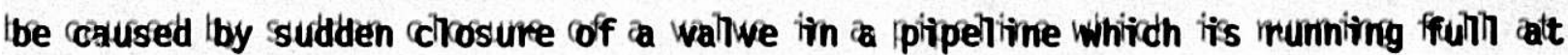

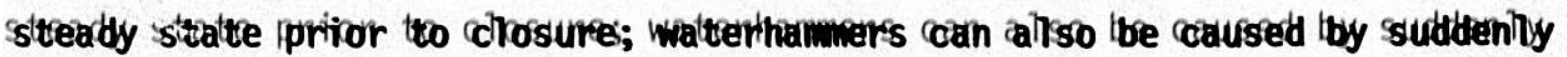

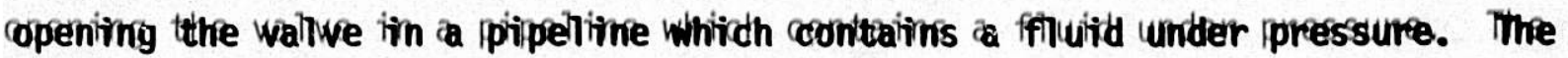

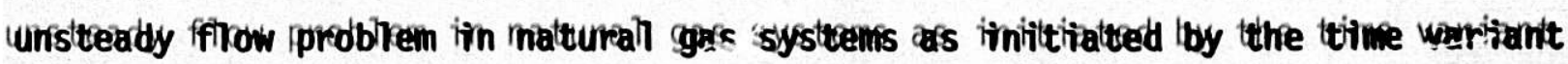

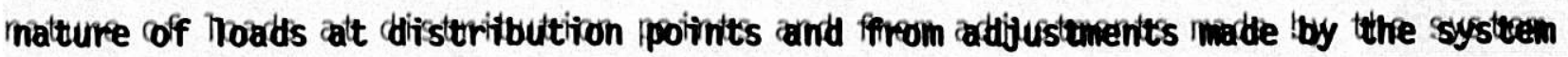

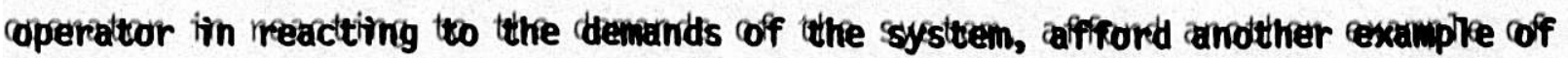

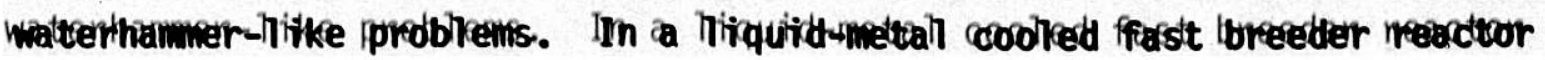




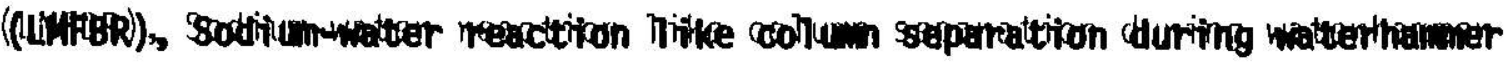

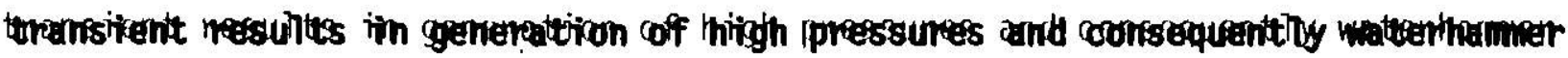
nitke toransiterntes.

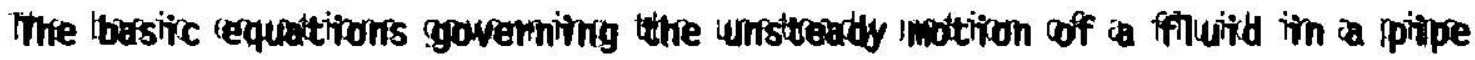

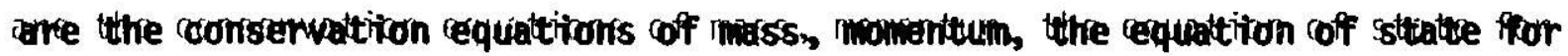

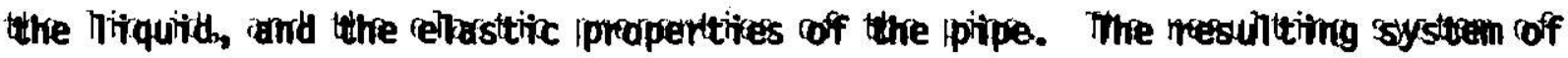

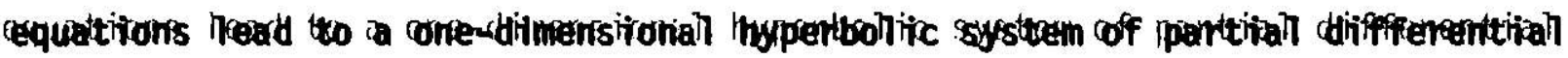

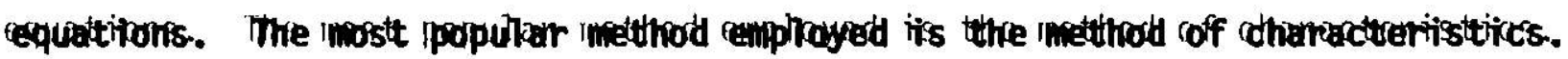

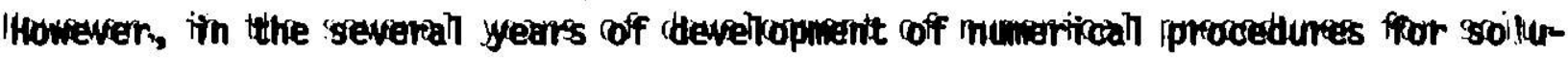

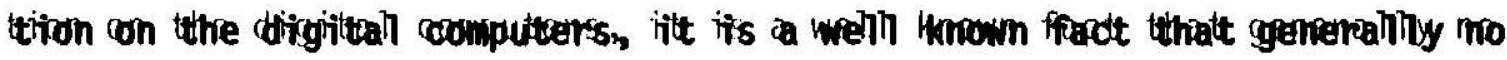

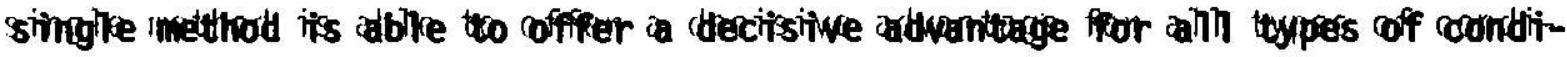

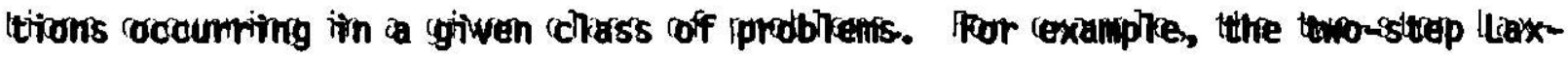

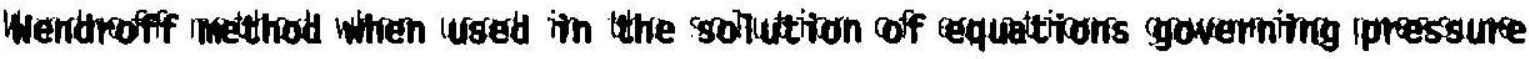

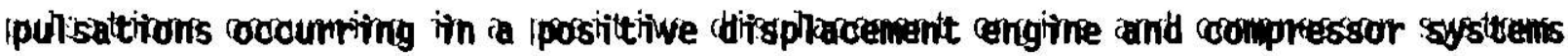

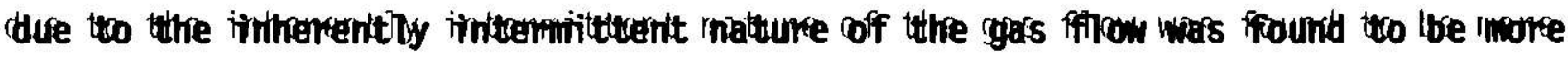

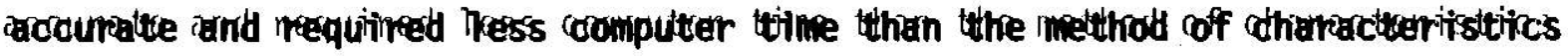

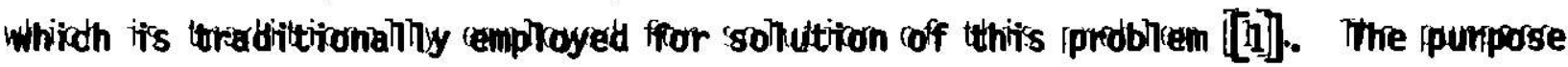

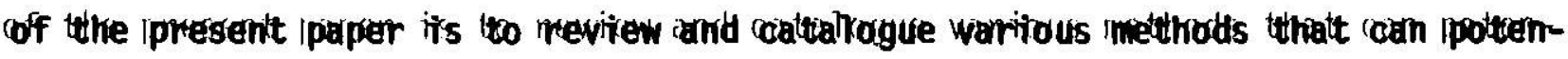

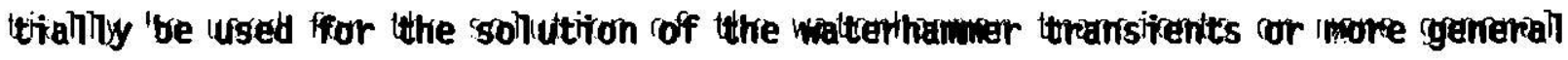

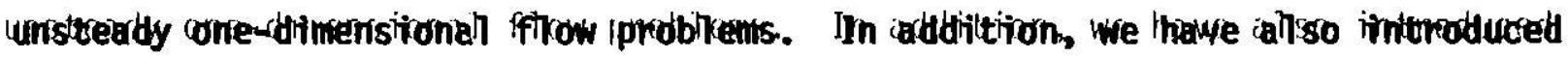

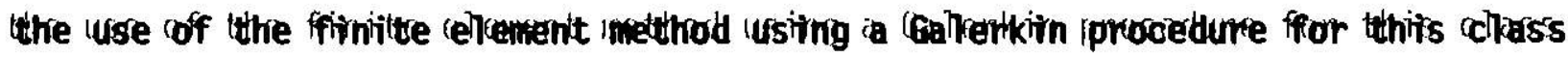

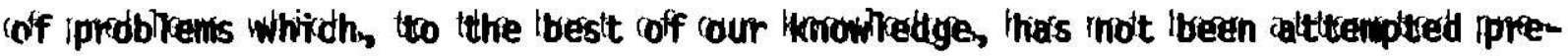

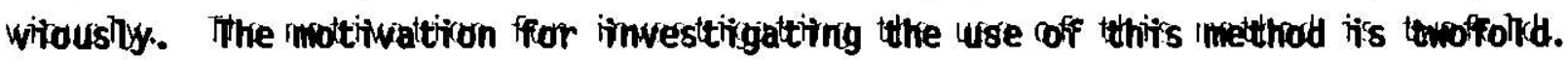

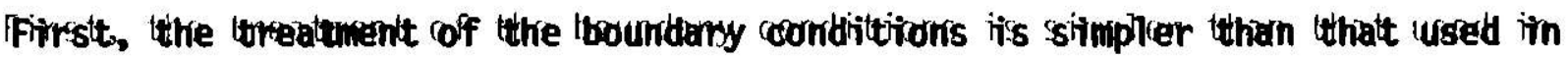

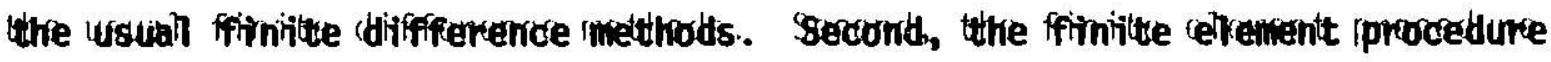

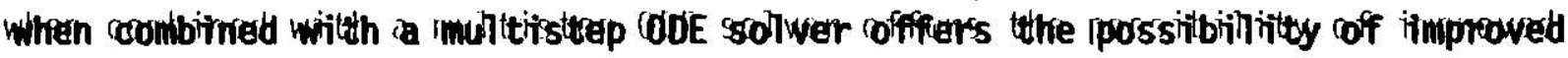

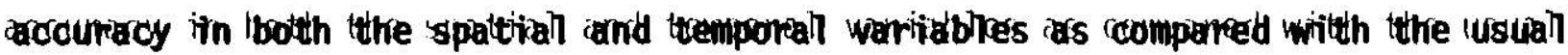

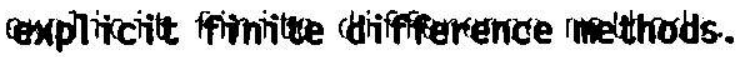

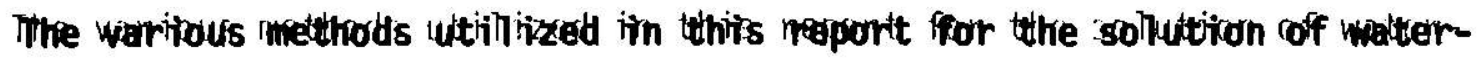
thawner toreansitientes ance

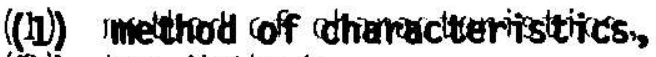

((2)) Lax Nethoth,

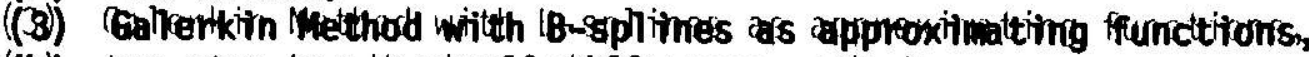

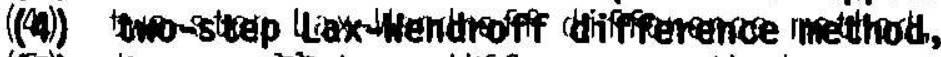

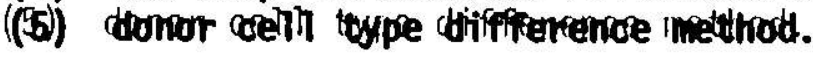

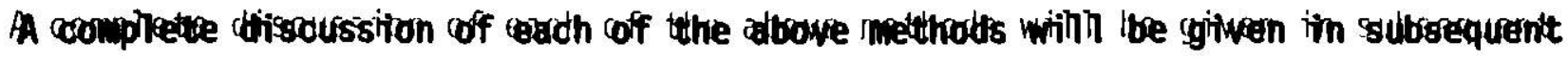
ssectritorist. 


\section{'2. GONERNING IEQUATIYONS IFOR WATERRHAMUER TTRANSIIENT'S}

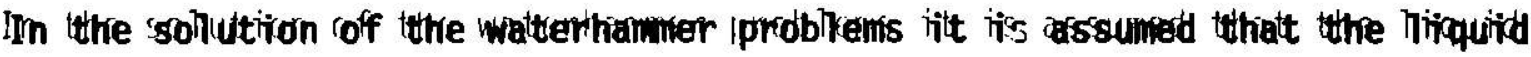

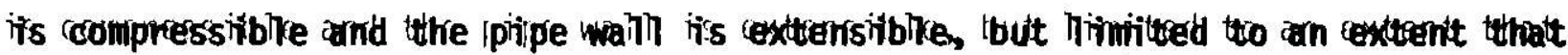

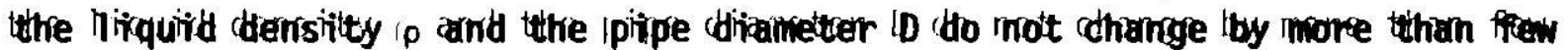

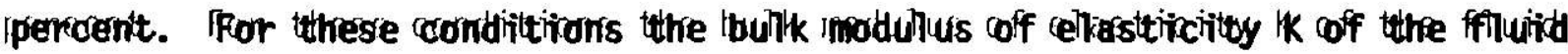

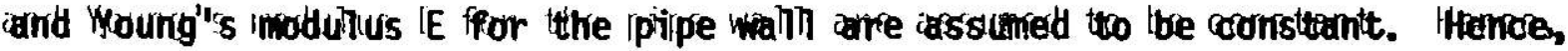

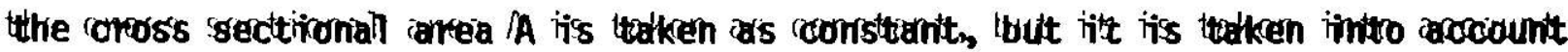

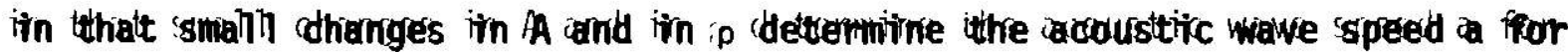

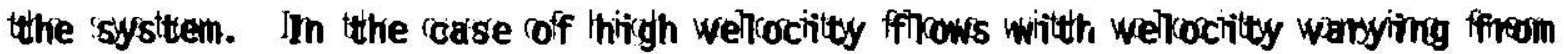

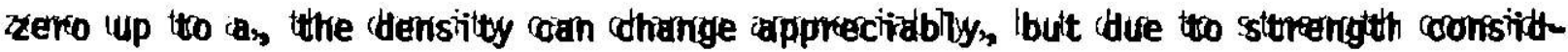

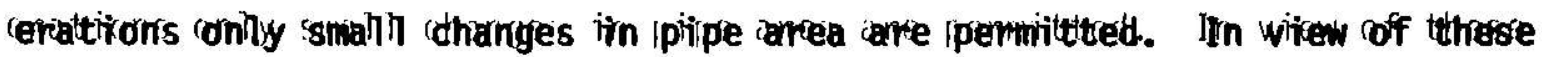

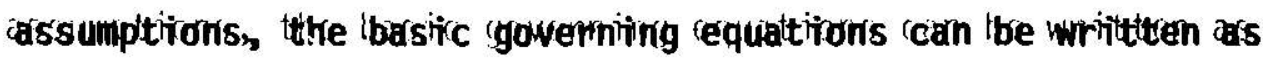

$$
\begin{aligned}
& \frac{\partial A_{\rho}}{\partial t}+\frac{\partial A_{p} u}{\partial x}=10
\end{aligned}
$$

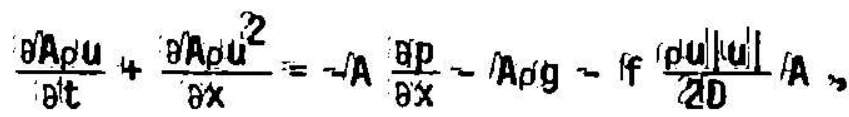

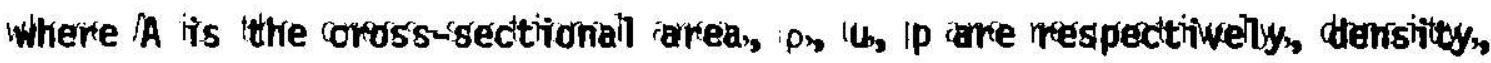

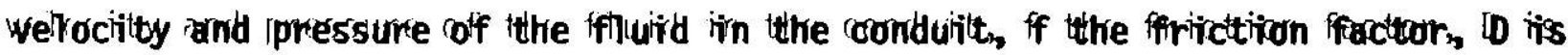

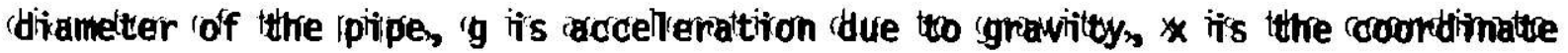
in the axivall diorection, and it its the thime.

IFrom the idefimilition cof lbuilk inodulus I $K$, we thave

$$
K=1 \rho \frac{d p}{d p}
$$

or

$$
K=\frac{i p}{i p}
$$

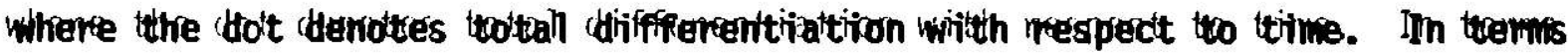

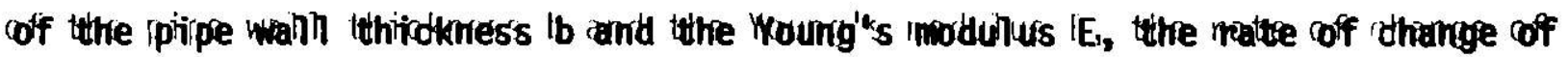
area for a thimatweth ipiipe its cgiven lby

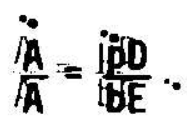

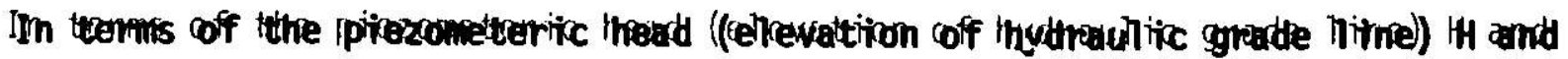

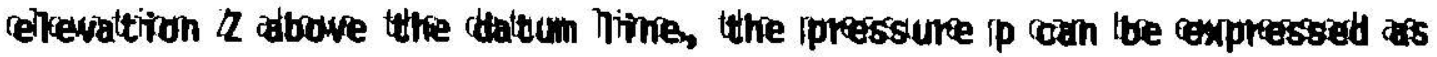




$$
P p=q_{R} g((H+Z Z)
$$

Where $o_{R}$ its a refterence deensiitey.

\subsection{The Carse of an Ellastio IPiipe lWalli}

Rrewrittiong LEq. ((2.11) a ds

$$
\frac{\partial \rho}{\partial t}+\frac{\partial \rho \partial u}{\partial x}+\rho \frac{\ddot{A}}{\frac{A}{A}}=0
$$

or

$$
\frac{\ddot{p}}{p}+\frac{\partial u}{\partial x}+\frac{\ddot{A}}{\not A}=0
$$

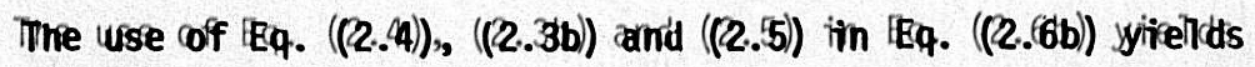

$$
\frac{\partial H}{\partial t}+u \frac{\partial H}{\partial x}+\frac{\partial^{2}}{\partial g} \frac{\partial u}{\partial x}=0
$$

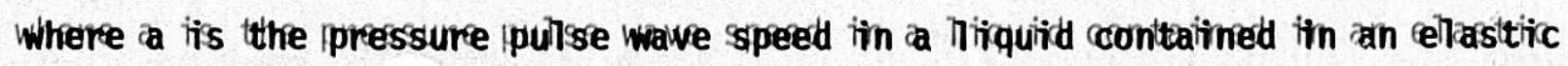
piipe and its deffimed tby the expressition

$$
a=\sqrt{\frac{1 / R / p_{R R}}{1+1 / K D / E b}}
$$

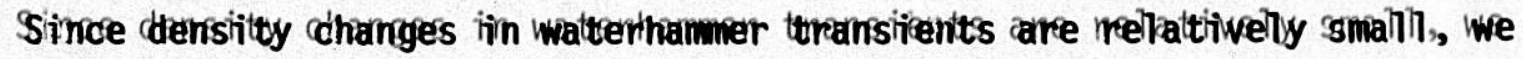
shalih therefore arssume thalt a its essentivialiny cornsitanit.

Rewriltiving Eqq. (12.2)) as

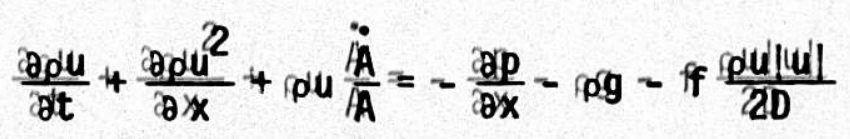

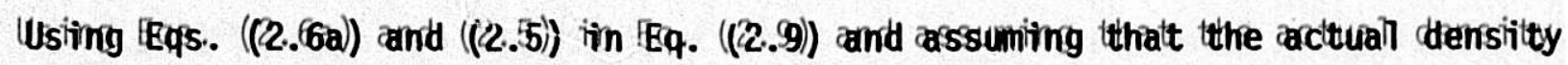

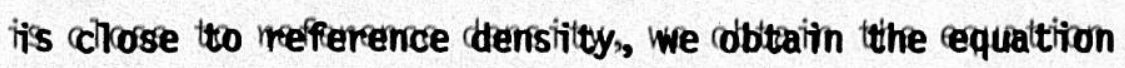

$$
\frac{\partial u}{\partial t}+u \frac{\partial u}{\partial x}+g \frac{\partial H}{\partial x}+f f \frac{u|u| l}{\partial D}=0 \text {. }
$$

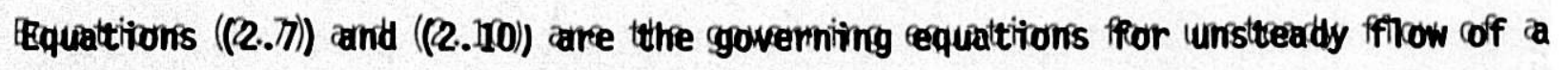

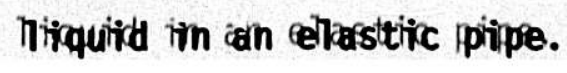




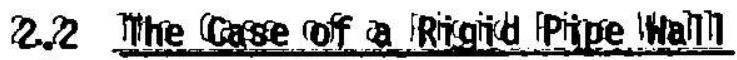

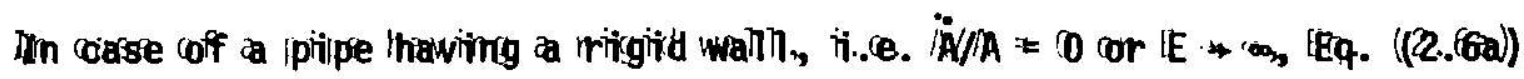
ssimplitifities too

$$
\frac{g \rho}{\partial x}+\frac{a \rho y}{\partial x}=0
$$

IEq. ((2.99)) reeducess tro

$$
\frac{\partial \alpha u}{\partial t}+\frac{\partial \alpha u^{2}}{\partial x}=-\frac{\partial p}{\partial x}-\log g-f f \frac{\partial u \mid u t}{\partial D}
$$

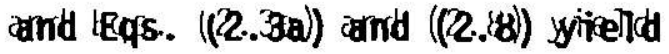

$$
d^{2}=\frac{K K}{i \rho}=\frac{d d p}{d d \rho}=q_{R}
$$

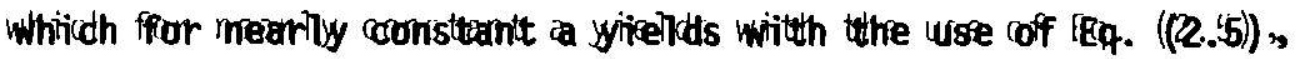

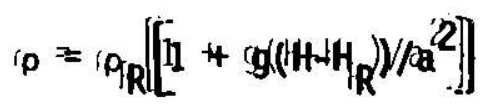

cor

$$
H=\frac{a^{2}}{q_{R} g}-C_{R}
$$

Where $c_{R}=a^{2} / M g-1 H_{R}$.

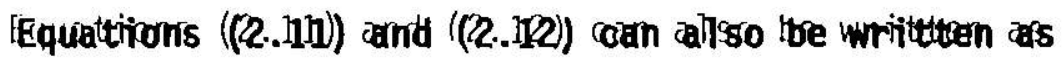

$$
\begin{aligned}
& \frac{\partial p}{\partial t}+\frac{\partial G}{\partial x}=10
\end{aligned}
$$

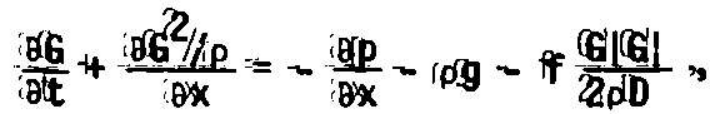

Where $6=i p t$.

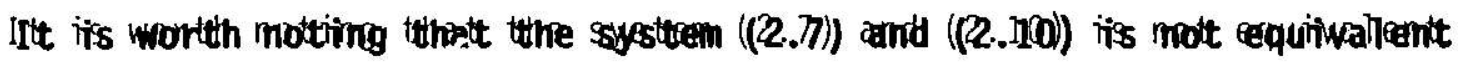

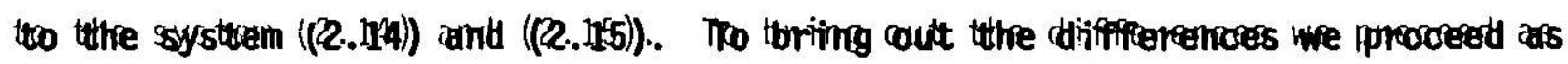

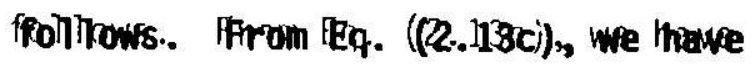

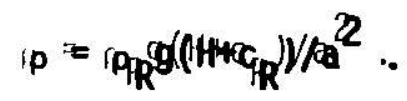


Thus

$$
\frac{\partial p}{\partial t}=\frac{p_{R} R^{g}}{a^{2}} \frac{\partial H}{\partial t} \text { and } \frac{\partial p}{\partial x}=\frac{p_{R} g}{\partial^{2}} \frac{\partial H}{\partial x} \text {. }
$$

IIt follitows that Eq. ( (2.174) thas the form

$$
\frac{\partial H}{\partial t}+u \frac{\partial H}{\partial x}+\frac{\partial^{2}}{\partial g} \frac{p}{\partial R} \frac{\partial u}{\partial x}=0 \text {, }
$$

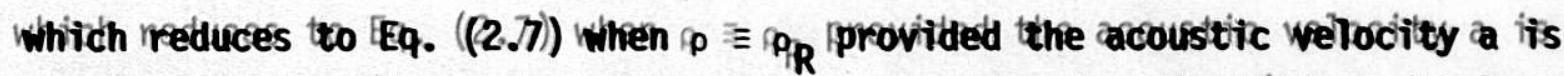

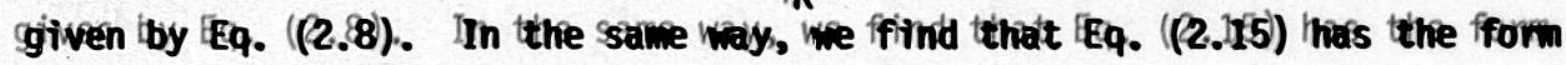

$$
\frac{\partial u}{\partial t}+u \frac{\partial u}{\partial x}+\frac{\partial R}{\partial p} g \frac{\partial H}{\partial x}+\frac{f f u \mid u l}{\partial \partial D}=0 \text {, }
$$

which its Eq. ( $(2.110)$ when $p_{p} \equiv p_{R}$.

\subsection{Anplication to a townstreath Walve Closure Probblem}

The demonstration problem consistes of a simple pipe systren with a reserwoir cupstrean and a wallwe downstrealn as shown ion ffilg. 2..1. The resenvoir

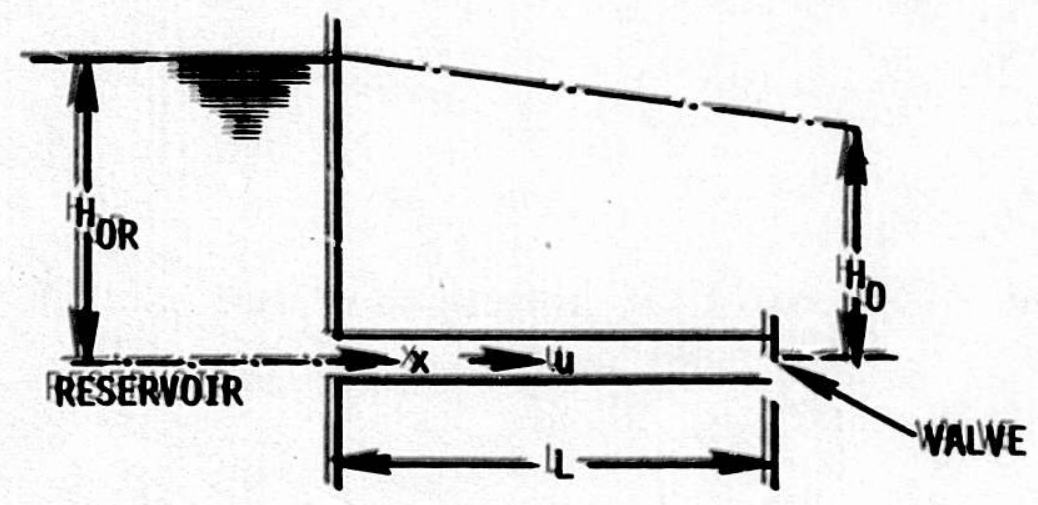

Fiig. 2.11 Dewornstration Probleen consitisting of a ssiuplie pipe systrem with a resenvoir lupstrean and a wallwe dowinstreath

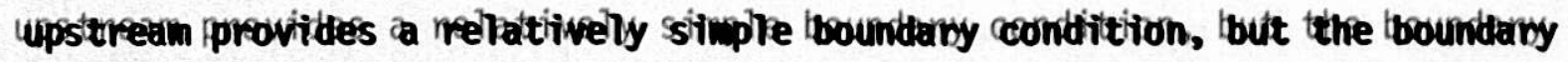

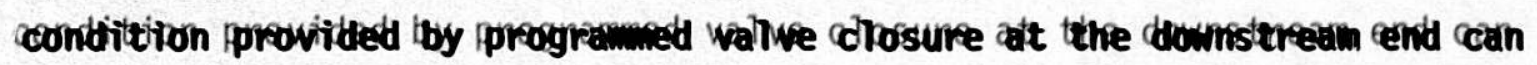

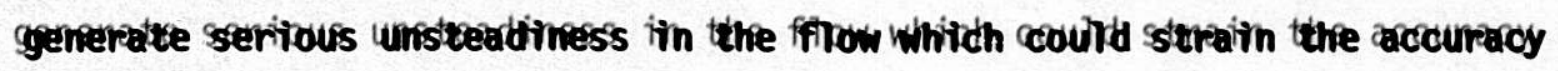
abtraimable with a given mumericall sscheme fror the ssolution of the governimg

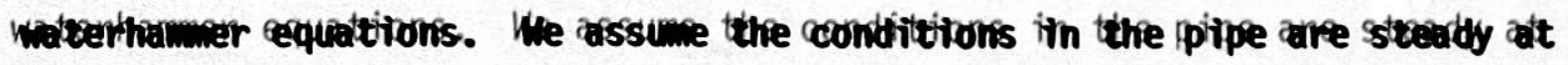

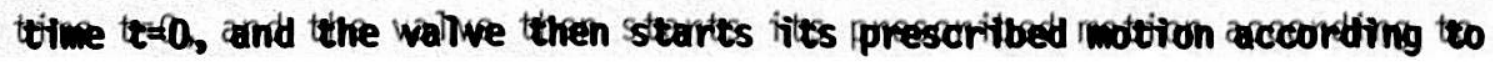




$$
\tau\left((t)=\left\{\begin{array}{cll}
\left.\left(1-t_{t} /(t)\right)^{m}\right)^{m} & \text { iff } & t<t_{c} \\
0 & \text { iff } & t \geq t_{c}
\end{array}\right\},\right.
$$

in which the is the tiime of cllosure, in iis a constant index which firixes the desired rate of chrosure. As is ccustomany, the wallwe its treated as an corififice and consequentlly the discharge ratte iis given as

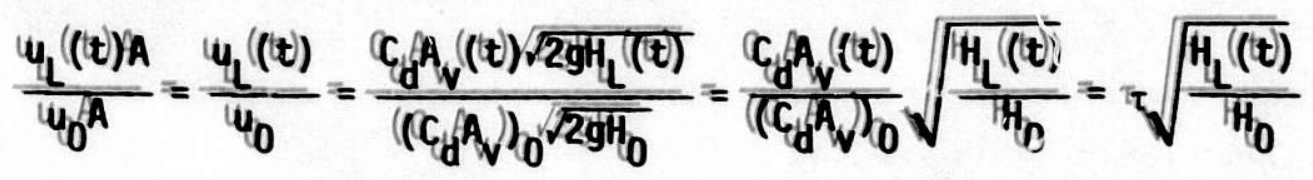

Where $H_{0}$ is the stready-state head hoss accross the wallwe and $y_{0}$ is the streadystate welocity in the pipe, $C_{C d}$ is the wallve-discharge coefficicient, $A_{v}(t)$ i is the area of walive copering, subscript zero denotes the strealy-statte conditionis,

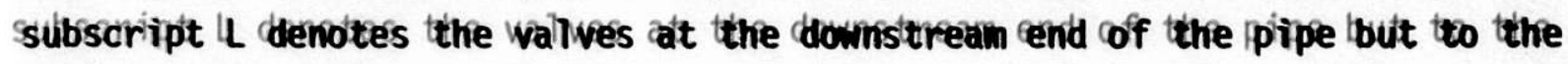
Theft of wallve and

$$
\tau=\frac{C_{d} A_{v}(t(t)}{\left(C_{d} A_{v}\right)_{0}} \cdot
$$

Equation ([2.177) trogether with EEq. ((2.116) providdes boundany conditition at the downstrealn eend of the lpipe; the wipstream boundany conditition fror lEqs. ((2.77) and $(2.110)$ with upstream reservoir is given

$$
H(0, t)=H_{O R} \cdot
$$

The boundany conditition for IEqs. ((2.174) and ((2.115) corresponiding too the albove equation is cobtained by use of Eq. ( (2.113b) as

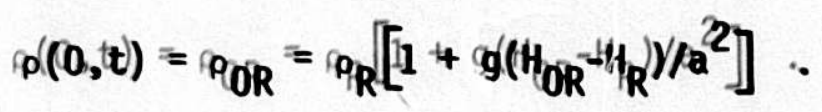

At how wellocitives the stready-statee solution can be given as

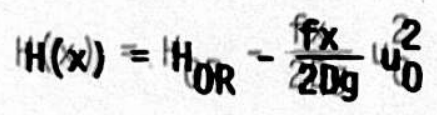

con,

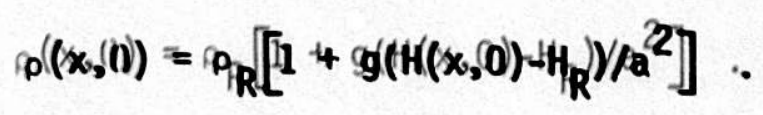

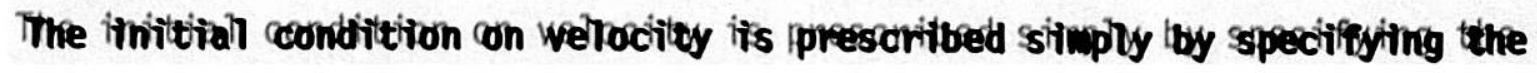


ithititial ssteady sstated fillow nate ais

$$
w(x, 0)=w_{0} .
$$

The specificic wallues of warious paraneters used in the mumericical ssolutionis tro be descritibed in severall stubsequent sections are $L=4253.5$ fit., $=3963$

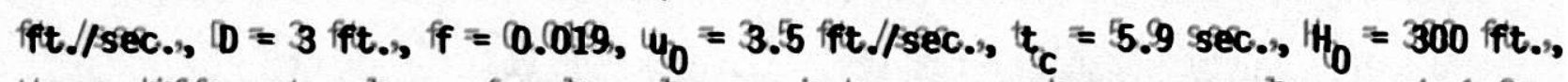

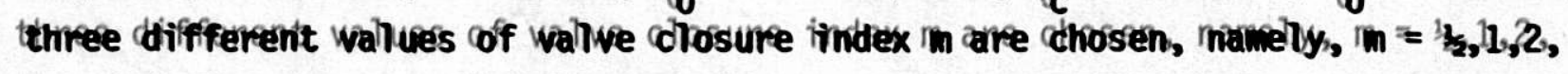
$H_{R}=3000$ fit. and $p_{R}=62.4 \mathrm{Th} / \mathrm{h} / \mathrm{cu}$. Fit. 


\section{METHOD OF CHAPAACTERISTIICS}

The monconservative form of EEq. ((2.7)) and the momentum Eq. ((2.110) form a paior of quasi-linear hyperboliic partiall differentiall equations in tho dependent wariables, thead th and welocitty w; and two independent wariables, distance allong the pipe $x$ and time $t$. The method of characteristios ((MOC) its a maturall choice for solving hyperbolic systems, because the governing equations are casit in a form that describes the condiitions allong curves callied characteristetics on which the physicical dissturbance travels.

\section{Characteristic Form}

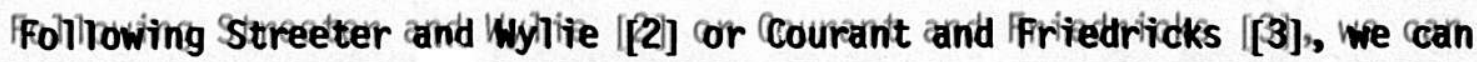
proceed as follhows. Leet $L_{1}$ denote the hefit hand sside of $\left[E q .((2.77))\right.$ and $L_{2}$ the Treft thand siide of EEq. ((2.110)). Then we form the Tiinear combination

$$
\lambda L_{L_{1}}+L_{L_{2}}=\lambda\left[\left[H_{t}+((g / \lambda)+\omega) H_{x}\right]+\left[\left[u_{t}+\left(\left(u+\frac{\lambda a^{2}}{g}\right)\right) u_{x}\right]+\frac{u(u) / f}{2 D}=0 .\right.\right.
$$

Choose $\lambda$ so thait each expression in the brackets is a divectionall derivative in the same dirrection. That iis,

$$
\begin{aligned}
& u_{t}+\left(\left(u+\frac{x a^{2}}{g}\right)\right) u_{x}=\frac{d d u}{d t}=u_{t}+\frac{d d x}{d t} u_{x}, \text { and } \\
& \left.H_{t}+((g / \lambda)+w) H_{x}=\frac{d d H}{d t}=H_{t}+\frac{d d x}{d t} H_{x} \text { on the cunve } x=x(t)\right) .
\end{aligned}
$$

Then

$$
\frac{d d x}{d t}=u+\frac{x a^{2}}{g}=u+g / d x
$$

Whirch givives

$$
\lambda= \pm g / \mathrm{ar},
$$

and the equations for the characteriistiocs

$$
\frac{d d x}{d t}=u \pm \pm \text {. }
$$

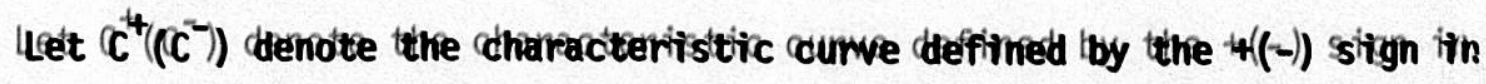

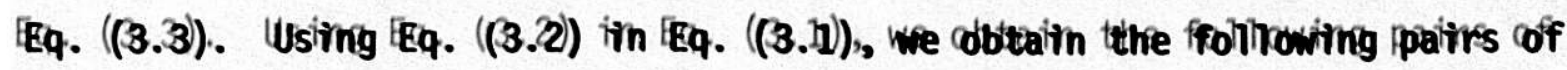
equatitions. 

$\left.\begin{array}{l}\text { a) } \frac{d d x}{d t}=u t+a \\ \text { b) } \frac{d g}{d} \frac{d t H}{d t}+\frac{d d u}{d t}+\frac{u(u) l f}{2 D}=0\end{array}\right\} \operatorname{con} c^{+t}$

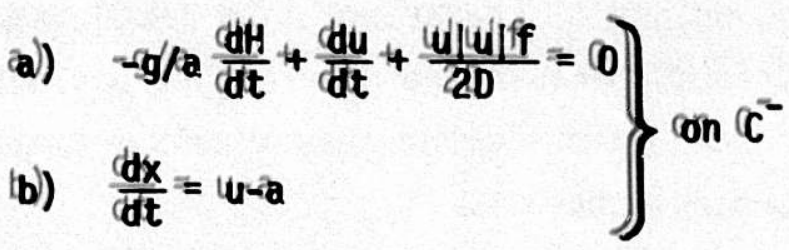

Method of Characterististics

In the method of characterisistics, the mumericall procedure sttants with the above system off cordinany diffiferentitial equationis. Suppose that alt time

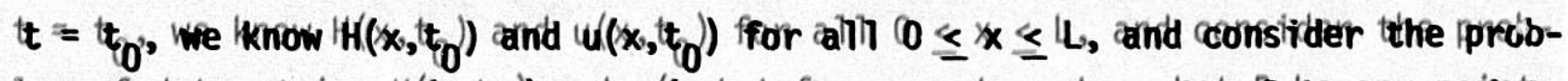

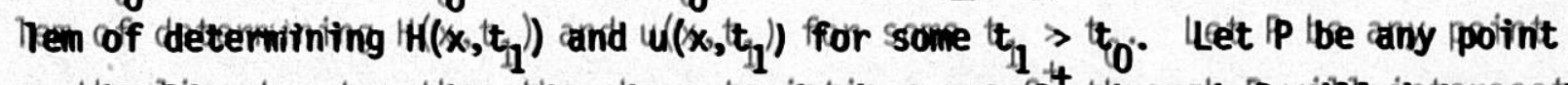
con the hime $t=t_{1}$; then the characteriistivc cunve $c^{+4}$ through $\mid \mathrm{P}$ willi intersect

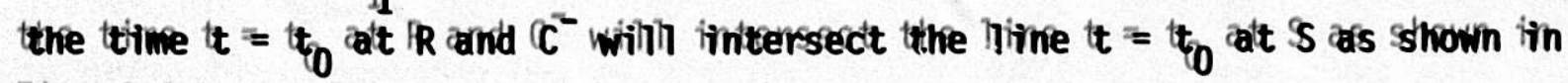
Fiig. 3.1.

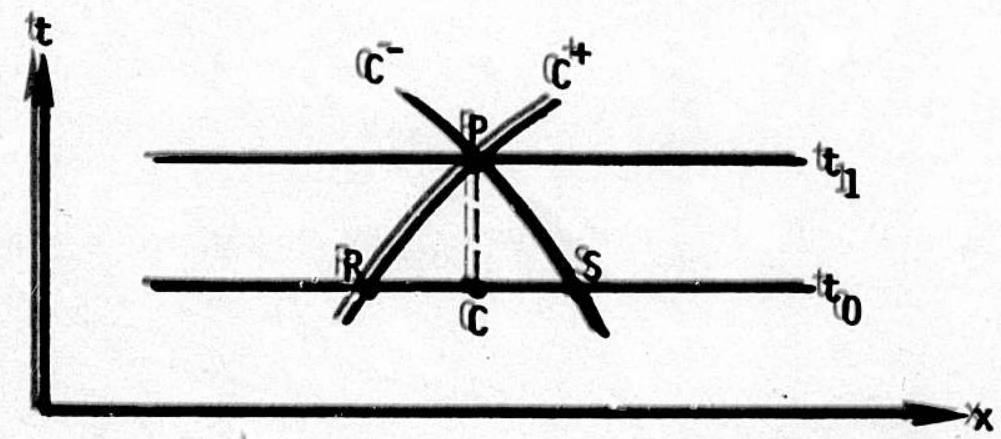

Firig. 3.11 IDisplay of the Characterisistitics

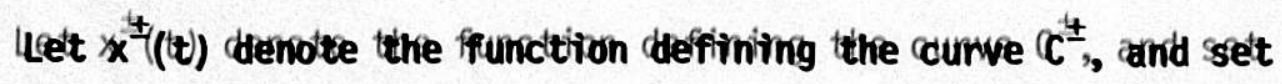

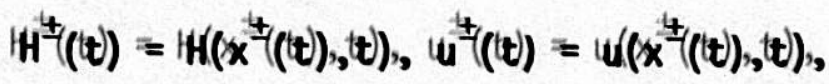

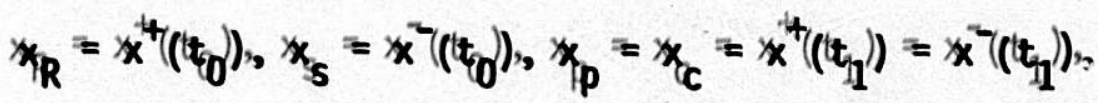

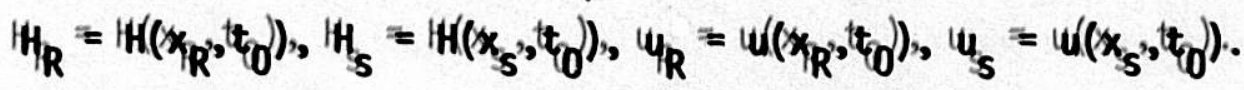

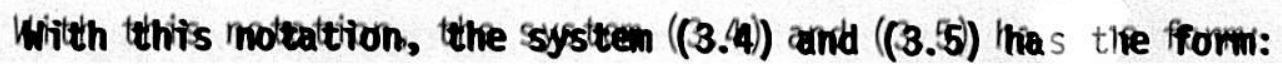



a) $\frac{d d x^{+}}{d t}=u^{+4}((t))+a$

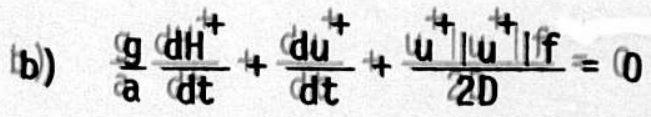
a) $-\frac{g}{d a} \frac{d H^{-}}{d t}+\frac{d u^{-}}{d t}+\frac{\left.u^{-} 7 / u^{-}\right] / f}{2 D}=0$
b) $\frac{d d x^{-}}{d t}=u^{-}((t))-a$

This system in turn is equivallent to the follhowing system of integral equations.

$$
\begin{aligned}
& \text { (ii) }) x_{p}-x_{R}=\iint_{t_{0}}^{t_{1}} 1\left(\left(u^{-t}((t))+a\right)\right) d t
\end{aligned}
$$

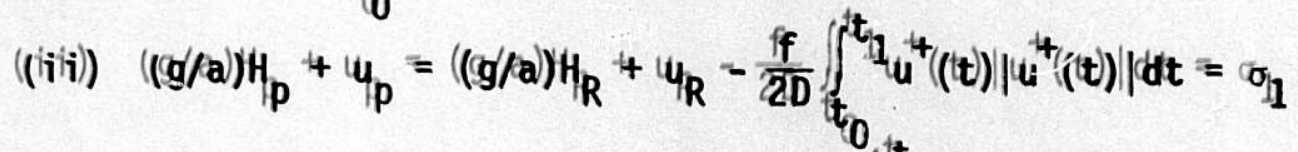

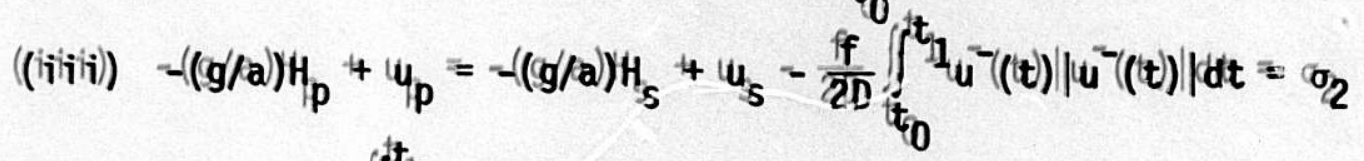

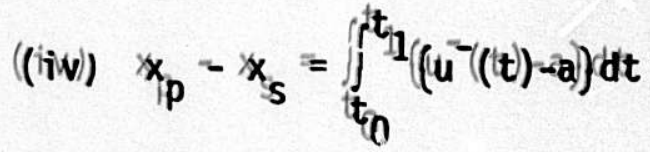

From the 2and and 3rd equation of the systtem, we fiind

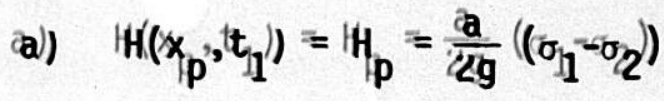

$$
\begin{aligned}
& \text { b) } \quad u\left(\left(x_{p}, t_{1}\right)\right)=u_{p}=\frac{11}{2}\left(\left(\sigma_{1}+t_{\sigma_{2}}\right)\right)
\end{aligned}
$$

\section{Approximate Equationis}

Up to thiis noinit no approximation thas tbeen made in deriving the systrem (13.8) and ((3.9)). There are sowo seapatte alpproximations which thave to the maide

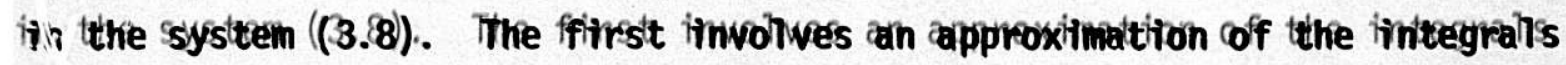
appearing in ((3.8)). The matture of thits approximattion ddetremines whether the

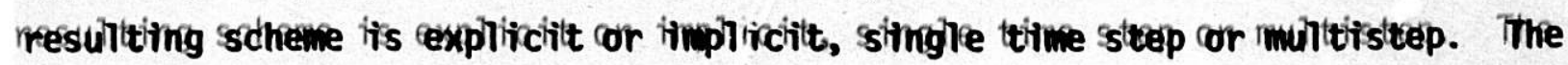

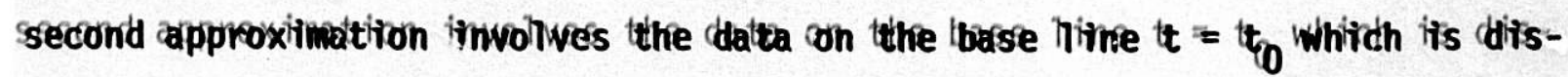

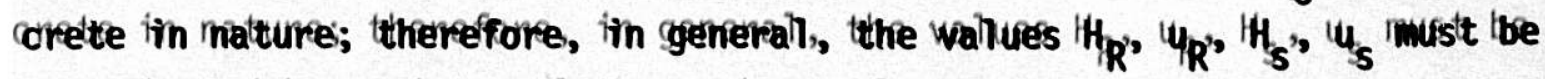
approximatted by an interpoliaterny sscheme of ssome teype. 
The frollhowing approximation is lused in evalhuating the integralis

$$
\left.u^{-4}((t)) \equiv u_{R}, 0 u_{s}, \int\right\} \quad t_{0} \leq t \leq t_{1} .
$$

This approximation heads to the follhowing expliocilt singlee strep scheme.

$$
\begin{aligned}
& \text { (ii) } x_{P}=x_{T_{R}}=\operatorname{sit}\left(\left(4_{R}+t^{+a)}\right)\right. \\
& \text { ((iii)) } x_{p p}-x_{s}=\operatorname{sit}\left(\left(u_{s}-a^{a}\right)\right. \\
& \text { ((iiiii)) } \sigma_{1}=\left(\left(g / a_{a}\right)\right) H_{R}+u_{R}=\frac{\delta(t f}{2 D} u_{R}|| y_{R} \mid \\
& \text { (iiv)) } \sigma_{2}=((-g g / a)) H_{s}+u_{s}-\frac{\delta i t f}{2 D D} u_{s}|| u_{s} \mid \\
& \text { (iv) } H_{p p}=\frac{a}{2 g}\left(\left(\sigma_{1}-\sigma_{2}\right)\right) \\
& \text { (wii)) } u_{p p}=\frac{11}{2}\left(\left(o_{1}+o_{2}\right)\right)
\end{aligned}
$$

\section{Boundary contilitions}

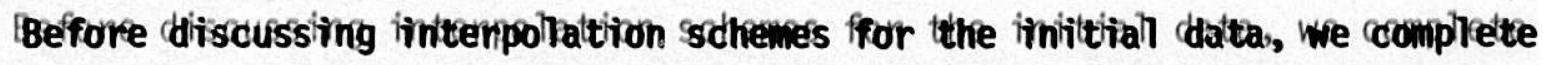

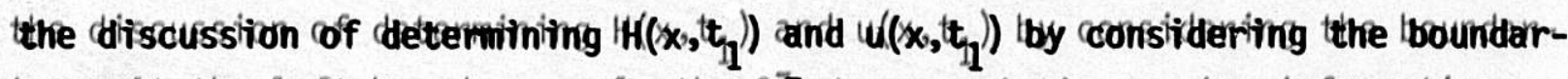

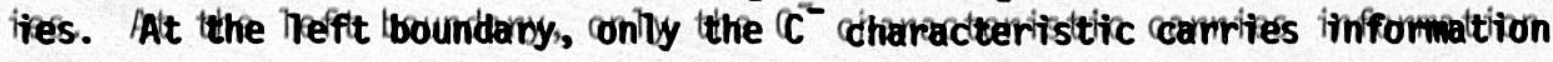

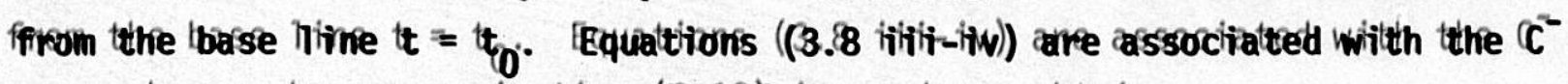

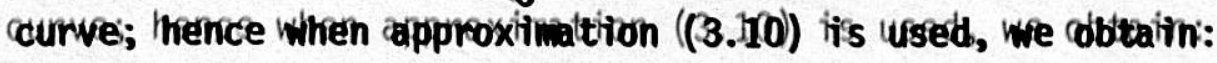

$$
\begin{aligned}
& \text { a) } x_{p p}-x_{s}=\operatorname{sit}\left(\left(u_{s}-x^{a}\right)\right) \text {, } \\
& \text { (b)) }((-g / f a)) H_{p}+u_{p}=((-g / f a)) H_{s}+u_{s}=\frac{f_{f}}{2 D D} u_{s}|| u_{s} \mid=\sigma_{2} \text {. }
\end{aligned}
$$

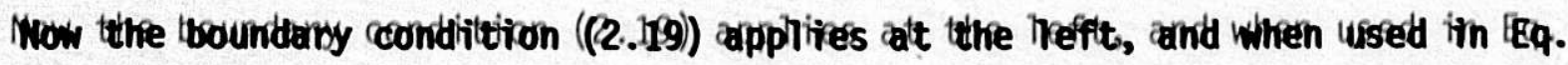
((3.112) yireldts:

$$
\left.y_{p}=(G / t a)\right) H_{O R}+\sigma_{2},
$$


where $\sigma_{2}$ its defined in ((3.12a)).

Con the riight boundary, conlyy the $C^{+}$characterisitic intersects the domain; thence when approximation $((3.10))$ iss lused in conjunction with $\left(\left(3.8 \mathrm{ij}-\mathrm{i}_{\mathrm{i} i \mathrm{i})}\right)\right.$, we frimd
a) $x_{p}=x_{R}=\operatorname{sit}\left(\left(u_{R}+a\right)\right.$
b) $\left.((g / / a)) H_{p}+u_{p}=(g / p a)\right) H_{R}+u_{R}-\frac{f f}{2 D D} u_{R}\left|u_{R}\right|=\sigma_{11}$.

In the present motation, the lboundany conditition (22.177)) on the riight takes the form

$$
u_{p}=\pi u_{0} \sqrt{h_{p} / H_{0}}
$$

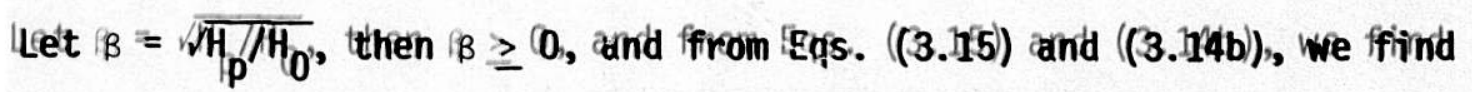

$$
\frac{g H_{0}}{a} B^{2}+\tau u_{0} B-\sigma_{1}=0
$$

which thas the uniqque mon-megativive noot $\left(\left(\sigma_{11} \geq 0\right.\right.$ from IEq. ((3.114b)))

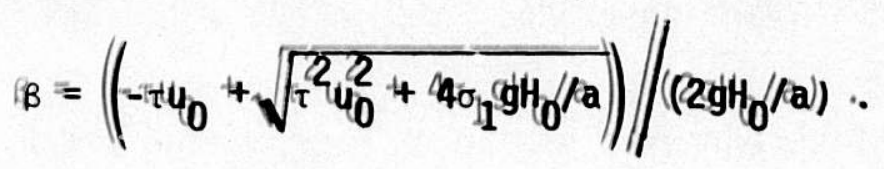

Hence
a) $H_{p}=H_{0} B^{2}$
b) $u_{p}=t u_{0}(E$

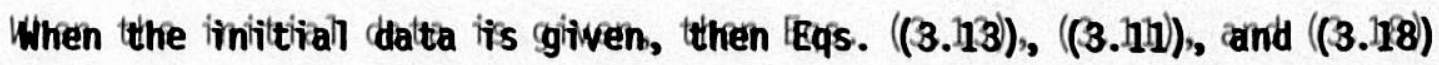

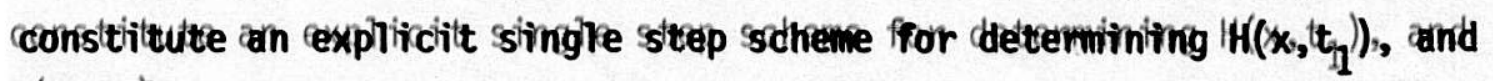
$u\left(\left(x_{0}, t_{1}\right)\right) \cdot$

\section{Intrenpoliation}

We mow consisider the ssecond approximation whith inwolwes the probblem of

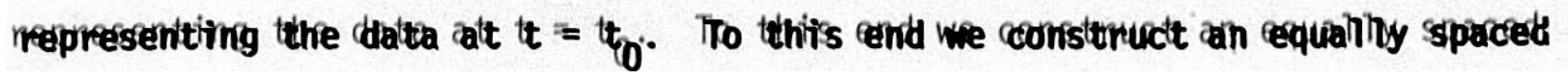


sgriid os the irittenwal] [[0,JL] lby settiting
a) $\quad i x=14 / N$, and

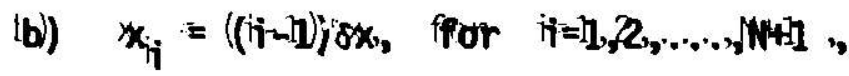

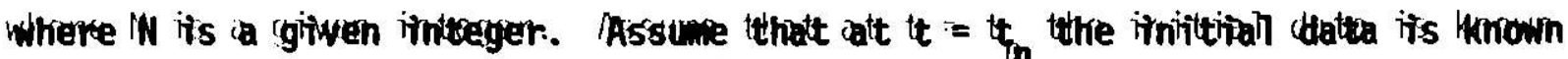

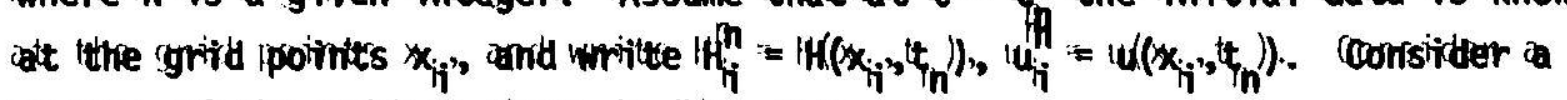
prorition of the grivid ars sthown in lfitg. 3.2.

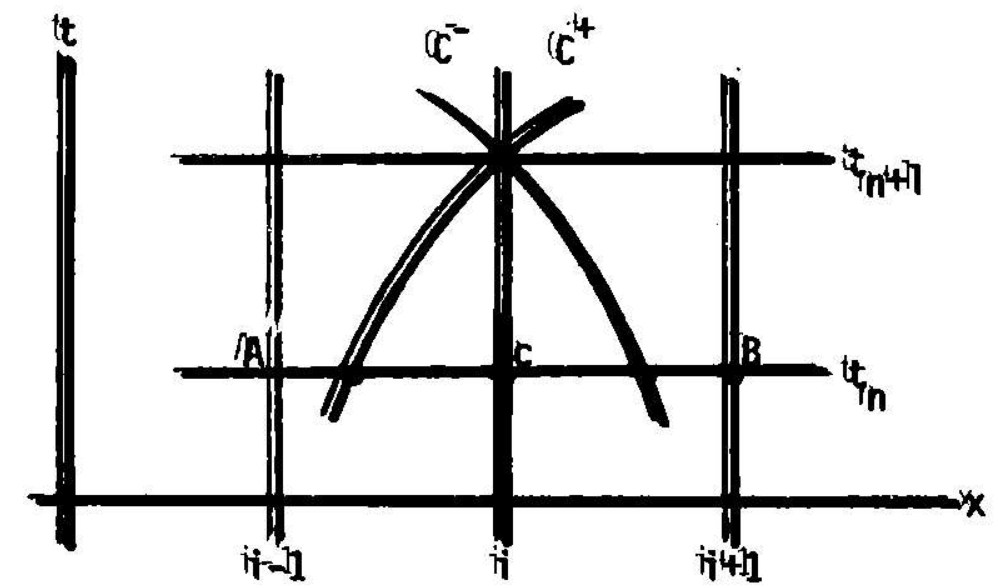

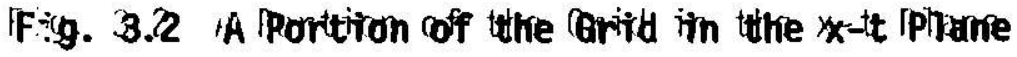

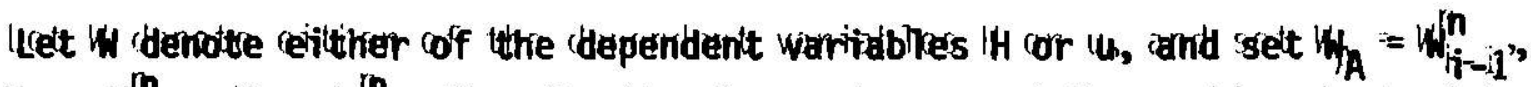

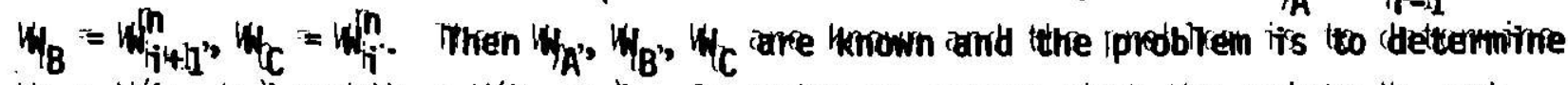

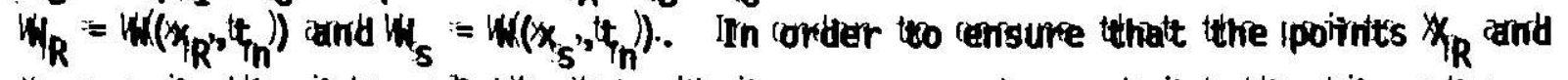

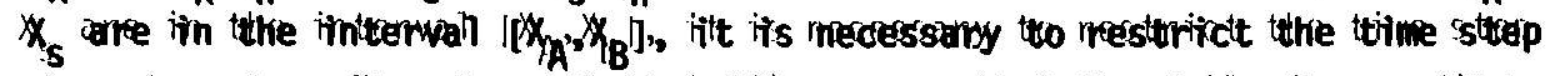

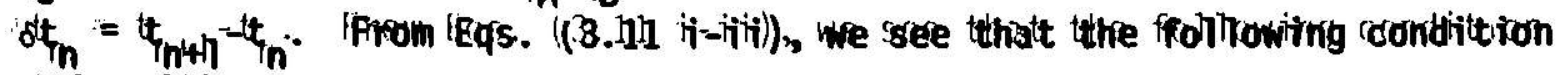
with suffifice

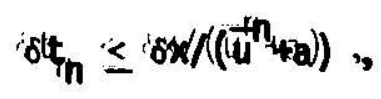

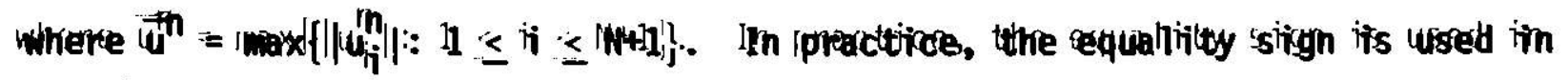
((3.20)).

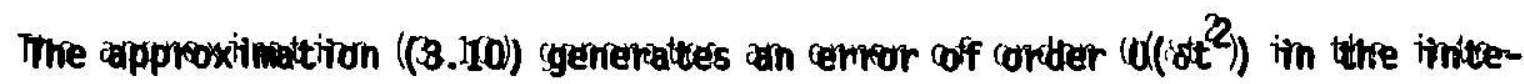

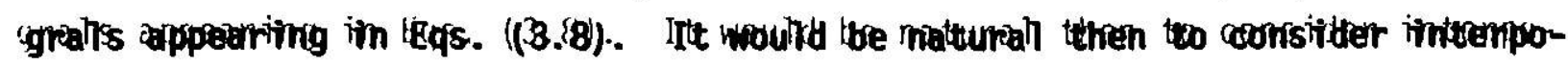

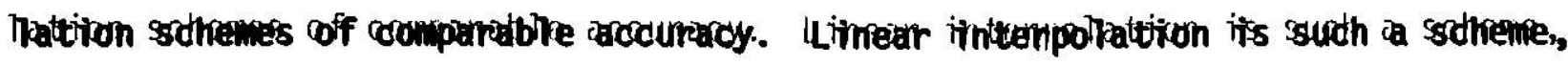


and we present ffirst the liinear initerpoliation scheme. We shall allso present a cquadrattic interpoliattion scheme; allthough we willi not consider a conrespondiing thiigher corder approximattion for Eq. ((3.110)).

\section{Liinear Interpolatition}

consiider the interpolvaltion of $\mathrm{U}$. From Fiig. 3.2., we ffind
a) $u_{R}=\left(\left(|| R C|| u_{A}+|| A R|| u_{C}\right) / / \delta x\right.$,
b) $u_{s}=\left(\left(|| C s\left|u_{B}+\right| j s B \mid u_{C}\right) / / \delta \delta x\right.$.

From Eqqs. ((3.1li i i-ijii )), we fiond

$$
\begin{aligned}
& || R C \mid=x_{p}-x_{R}=\operatorname{sit}\left(\left(y_{R}+a\right)\right), \\
& || A R \mid=\delta x-\cot t\left(4 R^{+a}\right) \text {, } \\
& \|\left(c s \mid=x_{s}-x_{p p}=\operatorname{sit}\left(\left(a-u_{s}\right),\right.\right. \\
& || s B \mid=\delta x=\operatorname{sit}\left(\left(a-u_{s}\right) ;\right.
\end{aligned}
$$

thus settining $\alpha=\delta t / \delta x^{2}$, we thave
a) $u_{R}=\left[\left[u_{C}+\operatorname{cor}^{2}\left(u_{A}-u_{C}\right)\right]\right] /\left[\left[11+\alpha_{C}\left(u_{C}-u_{A}\right)\right]\right.$,

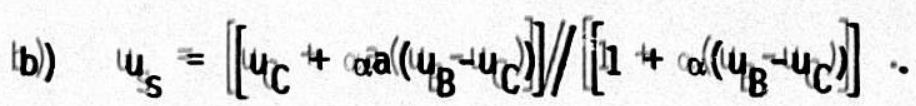

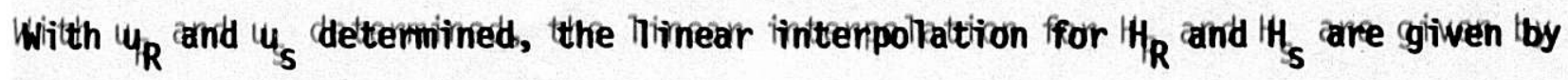

$$
\begin{aligned}
& \text { a) } H_{R}=\left(\left(|R R C| H_{A}+|\psi| A R \mid H_{C}\right) / / \delta x\right.
\end{aligned}
$$

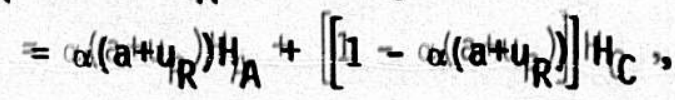

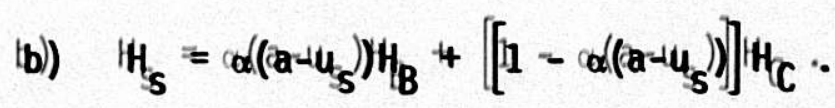

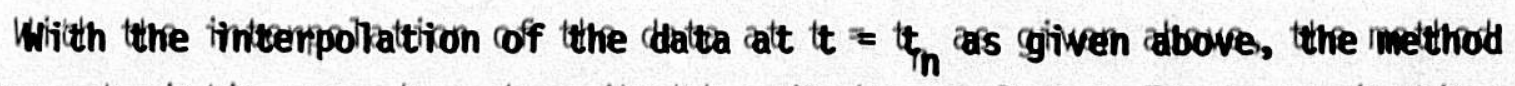

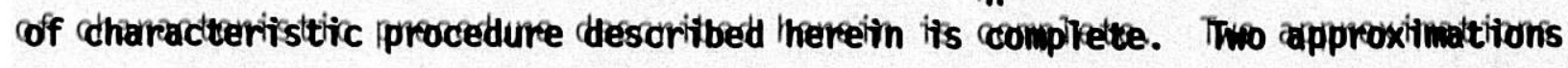

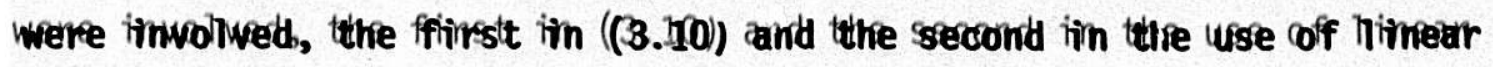




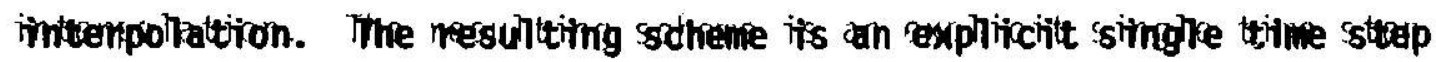
iprocedure.

\section{Quattrattic Ilntiterpollatioion}

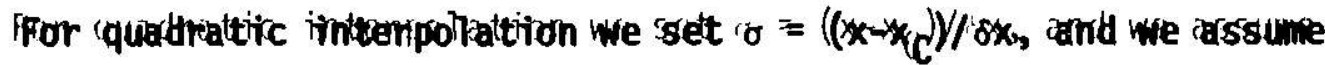

$$
W=A_{1} \sigma^{2}+A_{2} \sigma+W_{C}
$$

Where
a) $\quad A_{1}=\left(\left(w_{B}-2 / w_{C}+w_{A R}\right)\right) / 2$,
(b)) $\quad A_{2}=\left(\left(M_{B}-+W_{A}\right)\right) / R$,

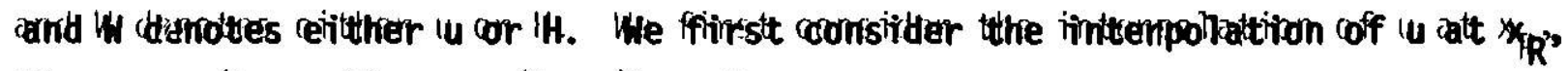

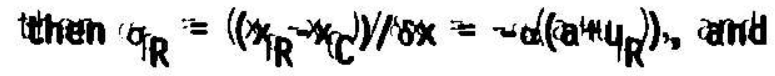

$$
\left.u_{R}=A_{11} a^{2}\left(\left(a^{2}+y_{R}\right)\right)^{2}-A_{2} \alpha\left(a^{2}+y_{R}\right)\right)+14_{C}
$$

cor

$$
A_{11} \alpha^{2} u_{R}^{2}+\left[\left[2 a A_{1} a^{2} \alpha^{2}-A_{2} \alpha^{\prime} \alpha-1\right]\right] \mid u_{R}+A_{11} a^{2} a^{2}-A_{2} a^{2 a}+1 u_{C}=10
$$

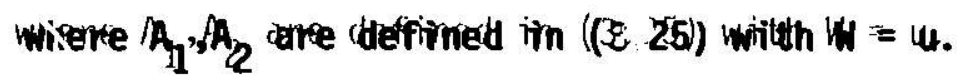

The difiscriiwimant cof thits requaltion its

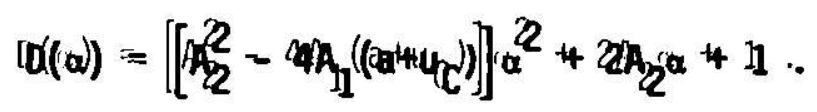

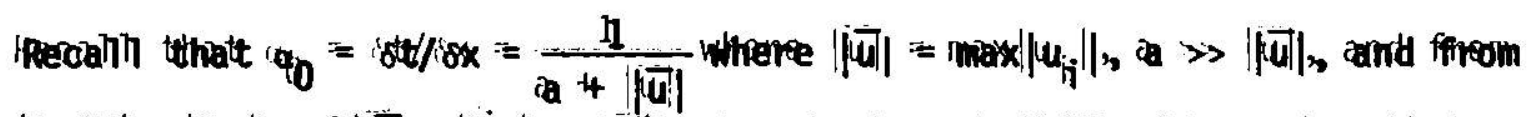

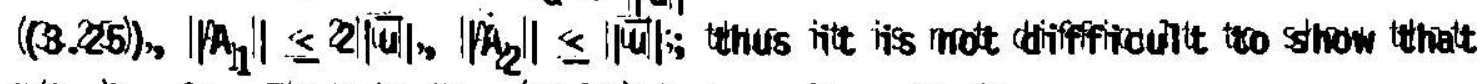

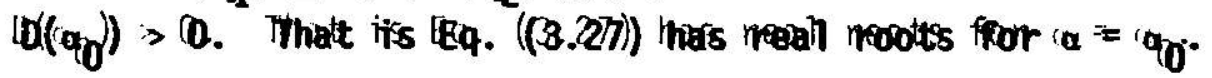


IIf we sett

$$
\begin{aligned}
& E_{11}=A_{1} \alpha^{2}, \\
& E_{2}=22 A_{1} a^{2} \alpha^{2}-A_{2} \alpha-11, \\
& E_{3}=A_{1} a^{2} a^{2} \alpha^{2}-A_{2} a \alpha+u_{C},
\end{aligned}
$$

then Eq. ((3.25)) thas the two rroots

$$
\begin{aligned}
& u_{R}^{((1))}=-22 E_{3} /\left(\left(E_{2^{2}}-\sqrt{(D)}\right),\right. \\
& u_{R}^{((2))}=-2 E_{3} /\left(E_{2}+\sqrt{D(D)}\right) .
\end{aligned}
$$

Since $\alpha=\frac{1}{a+|| \vec{u} \mid}$ and $a \gg|| \vec{u} \mid$, iitt frollirows thait $E_{2}<(0$; thuis

$$
\begin{aligned}
& u_{R}^{((1))}=22 E_{3} /\left(\left(|| E_{2} \mid+\sqrt{D}\right)\right. \\
& u_{R}^{((2))}=22 E_{3} /\left(\left(\left|E_{2}\right|-\sqrt{(D)}\right)\right.
\end{aligned}
$$

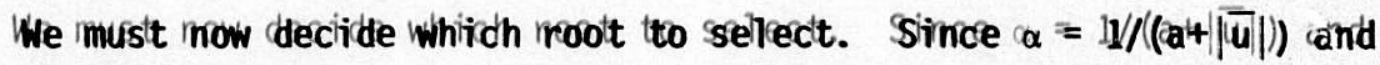
a $\gg|| \bar{u} \mid$ we know thatt $\alpha \ll 1$; t butt then for smalil $\alpha$ we have

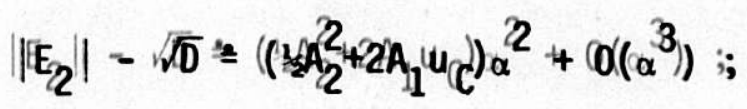

Whereas

$$
|| E_{2} \mid+110^{-} \div 2\left[\left[11+A_{2} \alpha^{\alpha}+0\left(\alpha^{2}\right)\right)\right] .
$$

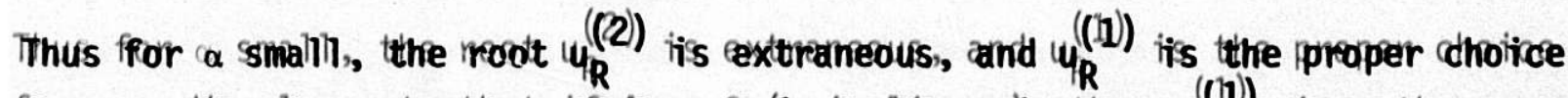
for $u_{R}$. We allso motte thalt iff $A_{11}=0((u$ iis inirnear $))$, then $u_{R}^{((1))}$ givives the correct

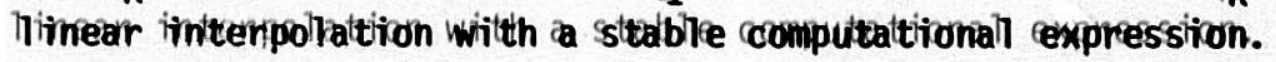

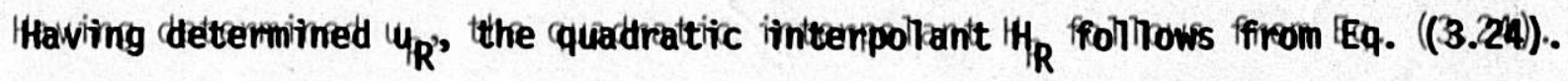

$$
H_{R}=H_{(C}-B_{2} \alpha\left(\left(\operatorname{ar}+y_{R}\right)\right)+B_{1}\left(\left(\operatorname{col}\left(\operatorname{ar}+y_{R}\right)\right)\right)^{2} .
$$




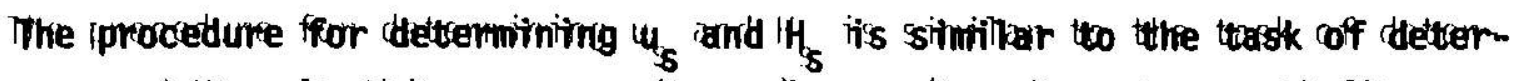

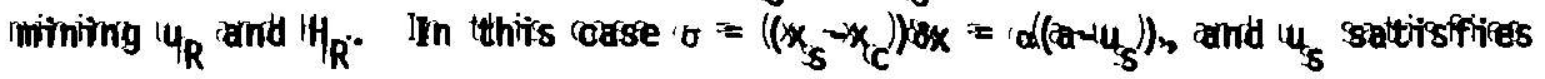

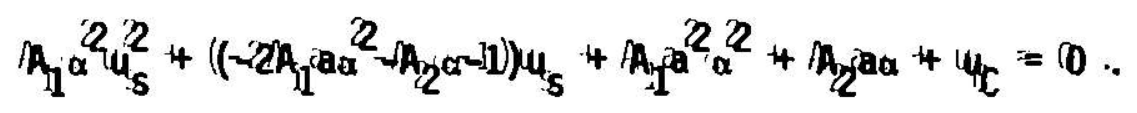

IIf we sselt

$$
\begin{aligned}
& \tilde{E}_{1}=/ A_{11} a^{2} \\
& \tilde{E}_{2}=22 A_{1} a^{2} \alpha^{2}-A_{2} \alpha-11 \text {, } \\
& \tilde{E}_{B}=A_{1} a^{2} \alpha^{2}+A_{2} a^{a} \alpha+4{ }_{c}
\end{aligned}
$$

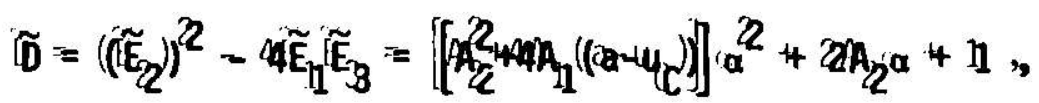

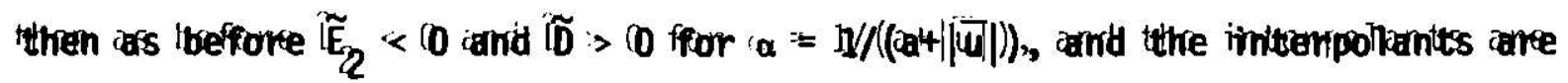

$$
\begin{aligned}
& \text { (a) } u_{\delta}^{((1))}=2 \tilde{E}_{3} /\left(\left(|| \tilde{E}_{2} \mid+1\right)\right.
\end{aligned}
$$

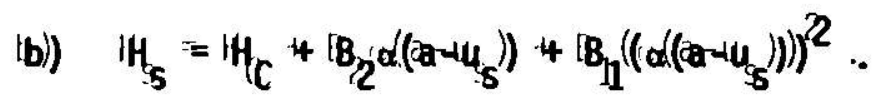

\section{Trumcattion Eemror and Stadbillitey}

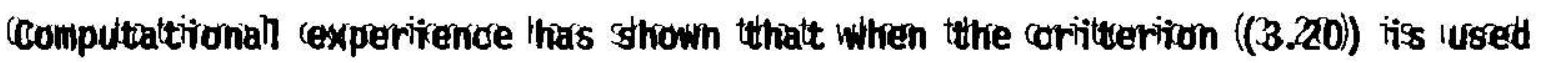

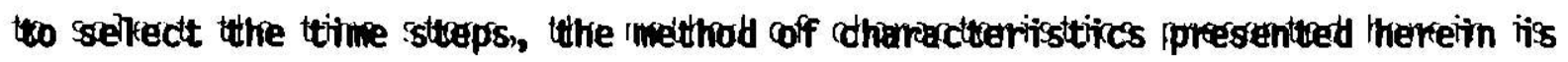

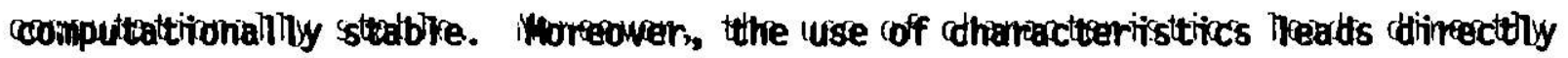

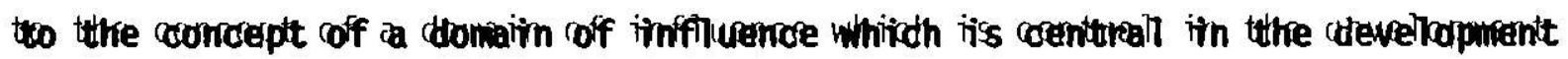

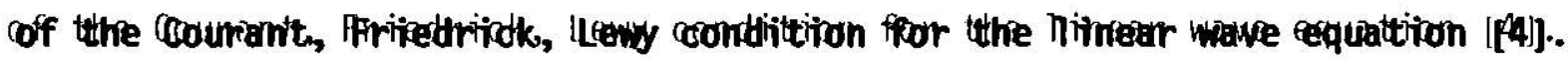

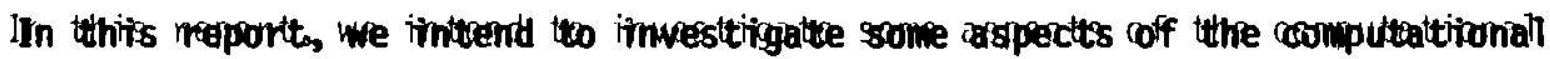

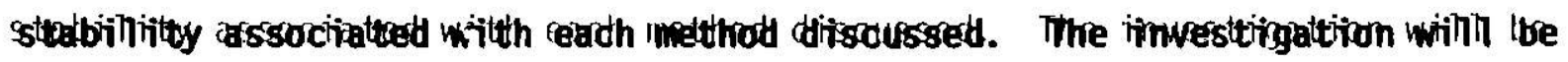

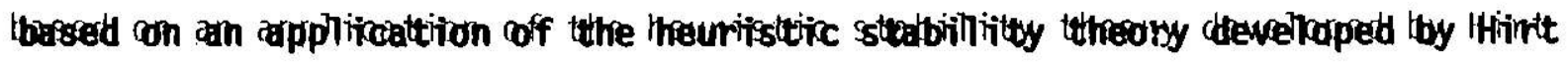

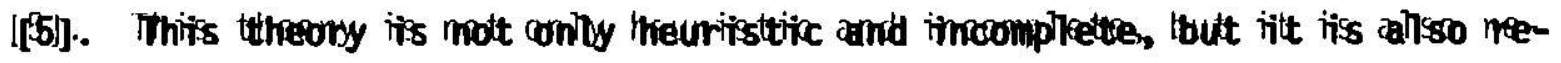

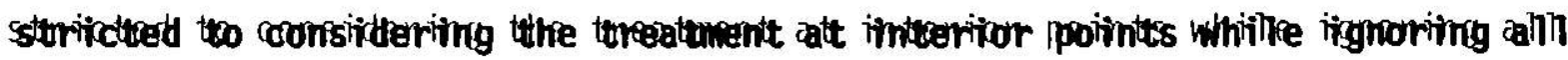

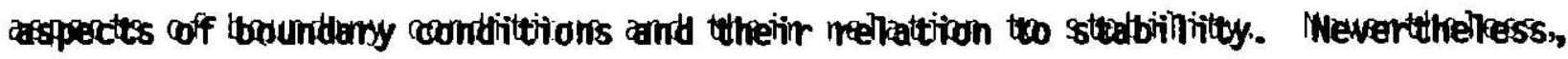

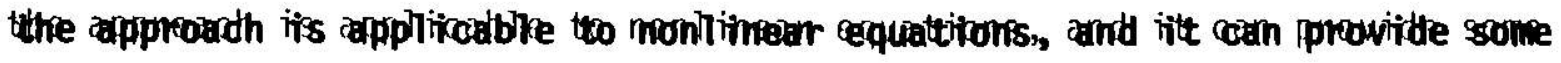

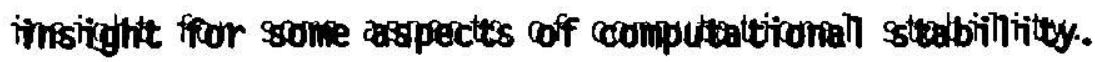

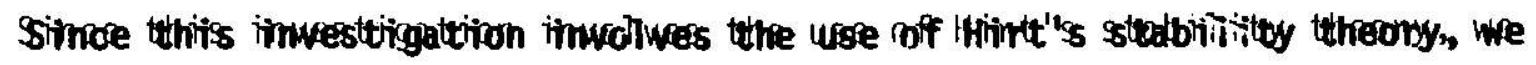

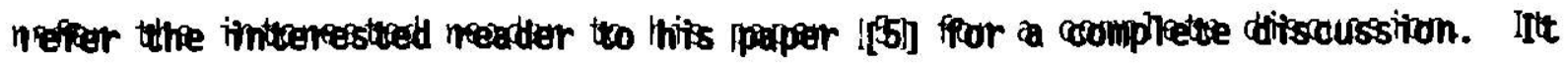


willil suffifice alt this point to summarize the basice iideas. The ffirsit sitep its to reduce the dissorete equaltion ((e.g. ffinitite dififference equaltion) to a diffifercentitiall equattion by expanding each tterm iin a Tayilor sserifes. For a consisistent approximation, the lrowesit corder tterms in the expansiton musit represent the corizginall diffiferentivall equattion. The thighter order tterms are the truncaltion

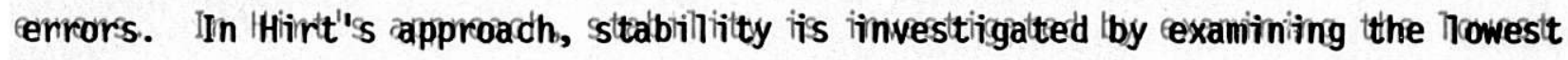
corder teruncaltion emrors. IIn particular, the lrowesit corder truncaltion enror

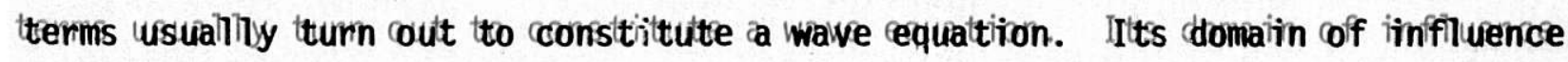
is then compared with the domaiin of infflurence of the disscrette equation. Some

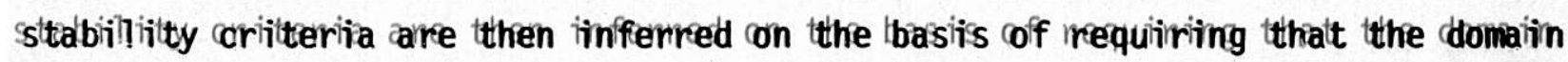
of imifluence for the dissorette equatition include the dromain of infiluence for the wave equation. The lrowesit corder teruncaltion emrors are then rewrittiten ion such a way that the corizginall equaltion together witth the lrowesit corder truncation

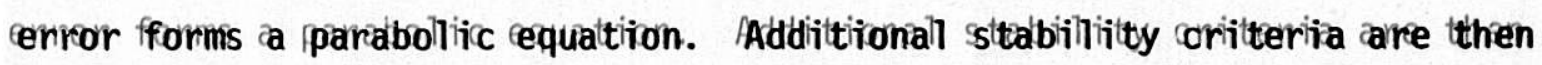
infferred by requiiring that the parabolitic equaltion its mathematicaliny sittablie.

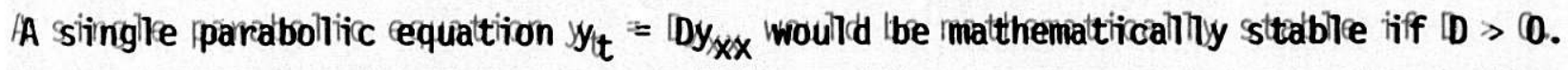

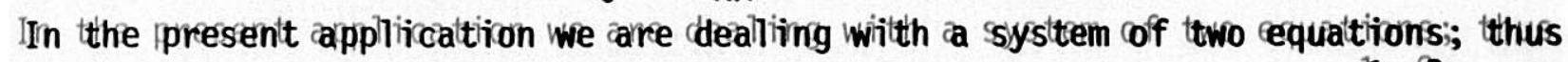
a iparaboliic teype equaltion would thave the form $w_{t}=\left[D W_{x x}\right.$ where $w=\left(\left(w^{1}, w^{2}\right)^{2}\right)^{\prime \prime}$ and

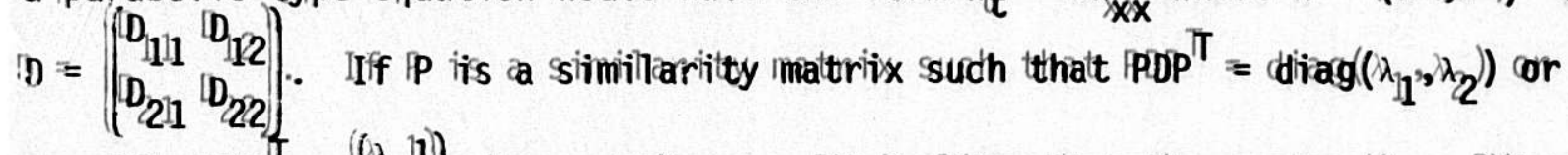

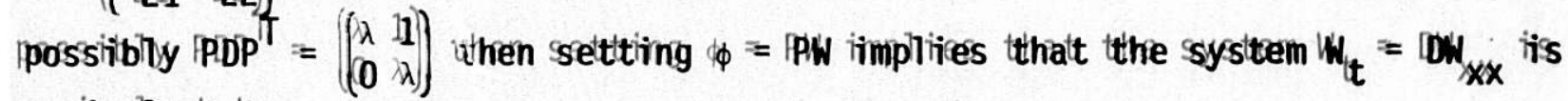
equivaliente to

cor

$$
\begin{aligned}
& \phi_{t t}^{11}=\lambda_{11} \phi_{x \times x}^{11}, \\
& \phi_{t}^{2}=\lambda_{2} \phi_{x \times x}^{2} ;
\end{aligned}
$$

$$
\begin{aligned}
& \phi_{i t}^{11}=x \phi_{x x x}^{11}+\phi_{x x x}^{2}, \\
& \phi_{i t}^{2}=x \phi_{x x x}^{2} .
\end{aligned}
$$

In eitther carse, iit its cllear thalt the syystem $W_{t}=$ IDW $_{x x}$ iis matthemalticcaliny siteablie

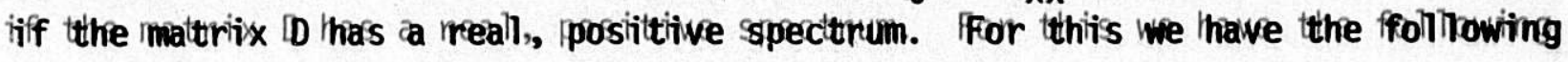
coritteriva: 
a) $\lambda_{i j}$ real i iffif $\left(\left(D_{111}-D_{22}\right)^{2}+4 D_{12} D_{21}>0\right.$.

(b) $\lambda_{i j}$ real iff $D_{11_{2}}$ and $D_{211}$ thave the ssame sign.

(c) IIf $\lambda_{i j}$ real, then $\lambda_{i j}$ its non-negative when $D_{111} D_{22}-D_{122} D_{21} \geq 0$.

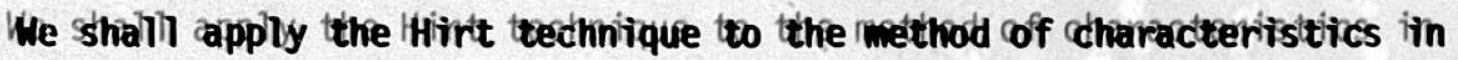
the case when himear interpolitation was used. We stint with EEq. ((3.22) for $u_{R}$ and $u_{s}$ expanding these cexpressions in a Tayilor serites about a teypicall

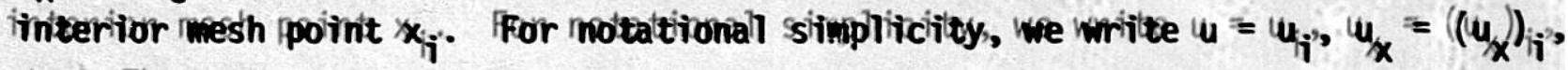
etc. Then

$$
\begin{aligned}
& u_{R}=\frac{u_{i}+\cos \left(\left(u_{i-1}-u_{i j}\right)\right)}{1+d\left(u_{i}-u_{i-1}\right)}
\end{aligned}
$$

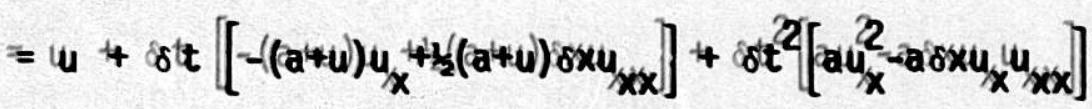

$$
\begin{aligned}
& +\cos \left(8 x^{2}, 8 t^{3}\right) \\
& u_{s}=\frac{u_{i j}+a d\left(u_{i+1}-u_{i j}\right)}{1+d\left(u_{i j+1}-u_{i j}\right)}
\end{aligned}
$$

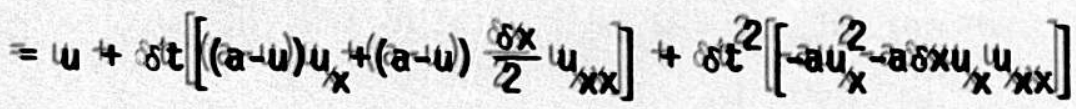

$$
\begin{aligned}
& \left.+\cos \left(\delta t^{3}, 8 x^{2}\right)\right) \text {. }
\end{aligned}
$$

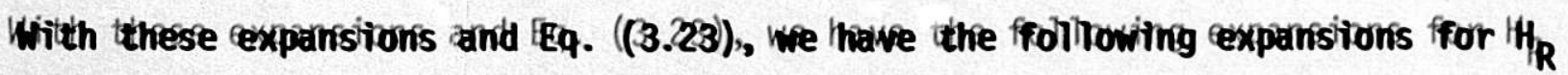
and $\mathrm{H}_{5}$.

$$
\begin{aligned}
& H_{R}=H_{i j}+\operatorname{col}\left(\left(a_{1}+y_{R}\right)\right)\left(\left(H_{i-1}-H_{i j}\right)\right)
\end{aligned}
$$

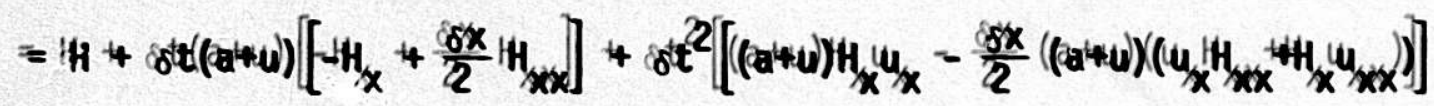

$$
\begin{aligned}
& +\cos \left(\cot ^{3} t^{3}, 8 x^{2} x^{2}\right)
\end{aligned}
$$




$$
\begin{aligned}
& H_{s}=H_{i j}+\alpha\left(a-u_{s}\right)\left(\left(H_{i j+1}-H_{i j}\right)\right.
\end{aligned}
$$

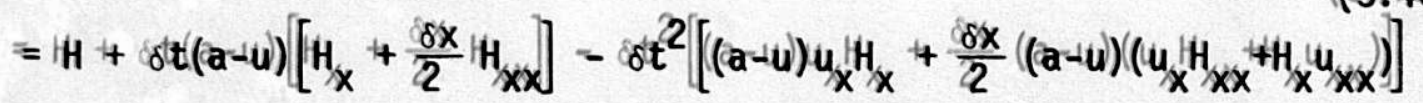

$$
\begin{aligned}
& \left.+\cos \left(\delta t^{3}, \delta x^{2}\right)\right)
\end{aligned}
$$

Now

$$
\begin{aligned}
& \text { a) } \left.H_{i}^{n+1}=H+\delta t H_{t}+\frac{\delta t^{2}}{2} H_{t t}+\alpha\left(\delta t^{3}\right)\right) \\
& \text { b) } u_{i j}^{n+1}=u+\delta t u_{t}+\frac{\delta t^{2}}{2} u_{t t t}+\alpha\left(\delta t^{3}\right) ;
\end{aligned}
$$

thus, iff we use the above cexpansions in Eqis. ((2.11l, ijiii-vii)), we fiind, affter some allgebra,

$$
\begin{aligned}
& H_{t}+u_{t x}+\frac{a^{2}}{g g} u_{x}=-\frac{\delta t}{2} H_{t t t}+\frac{\partial \sigma x}{2} H_{x x x}+\frac{\partial u}{2 g} d x u_{x x x} \\
& +\delta t u_{x} / H_{x}+\delta t \frac{a^{2}}{g}\left(\left(u_{x}+\frac{f}{D}\right) u_{x}+\alpha\left(\delta t \delta x, \delta t^{2}, \delta x^{2}\right)\right) \\
& u_{t}+u u_{x}+g g H_{x}+\frac{f f u|u|}{2 D}=-\frac{\delta t}{2} u_{t t}+\frac{\delta x}{2} \frac{g u}{2} H_{x x}+\frac{a \delta x}{2} u_{x x}
\end{aligned}
$$

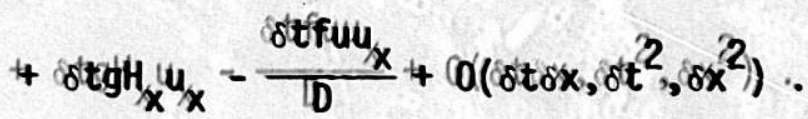

If we izgnore the thizgher corder teruncaltion error teemis in IEqs. ((3.42) and ((3.43)), we have a pair of second corder partivall dififferentivall equations. We

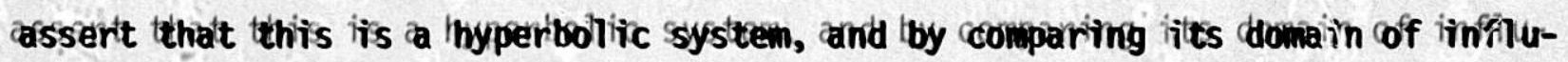
ence with the domain of inflinence of the dirscrette system ((3.11)), we will f fint the firsit stabilitity coritterion. To ffind the characterisitiocs of this system, we writte the systeen $(3.42-3.43)$ in the from

$$
\begin{aligned}
& \text { a) }-h_{t t t}+a H_{x x}+\frac{a u}{g} u_{x x}+\ldots=0 \\
& \text { b) }-u_{t t t}+a u_{x x}+\frac{u g}{a} H_{x x}+\ldots=0
\end{aligned}
$$

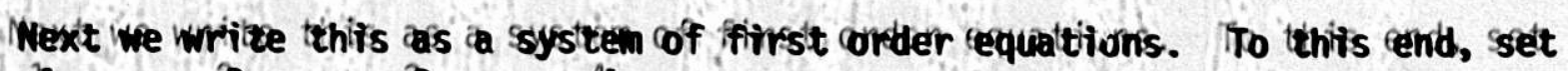
$\phi=H_{t}, \phi^{2}=H_{x}, \phi^{3}=u_{t}, \phi^{4}=u_{x}$, then $((2.44))$ hais the fromi: 


$$
A \phi_{t}+B \phi_{x}+\ldots=0
$$

Where

$$
\begin{aligned}
& A=\operatorname{diag}[-\infty, 1,-\infty, 1]] \\
& B=\left[\begin{array}{cccc}
0 & 0 & 0 & a u / / s \\
-1 & 0 & 0 & 0 \\
0 & \text { urg/ar } & 0 & 0 \\
0 & 0 & -11 & 0
\end{array}\right]
\end{aligned}
$$

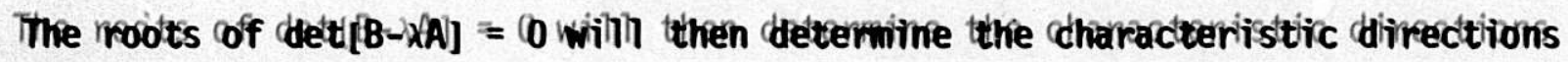
for the system $((3.45))$. Now

$$
\operatorname{det}\left[(B-x)=a^{2} \lambda^{4}-2 a d \lambda^{2}+a^{2}-u^{2}=0 ;\right.
$$

thence

$$
\lambda^{2}=\frac{a \pm|| u \mid}{a}
$$

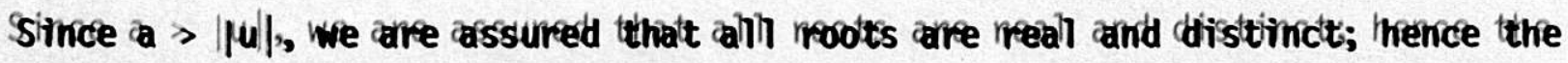
syysteen ios thyperbolitic, and the frour rootts are

$$
\lambda= \pm \sqrt{\frac{a \pm|| \omega \mid}{a}} .
$$

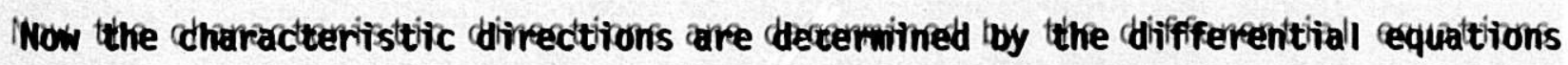

$$
\frac{d x}{d t}=\lambda \text {, }
$$

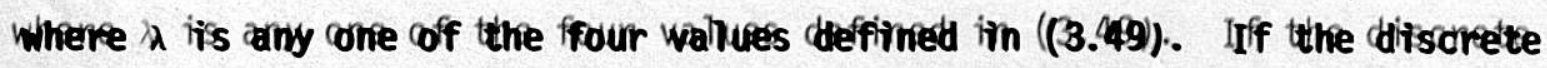
equation (3.113) iis tro have ssolutitions approxinationg the ssolutitions of the hyper-

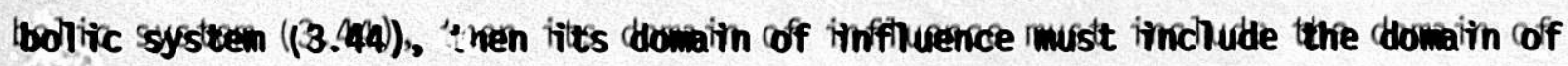
infifluence of $((3.46)$ ). That its

$$
|| x|=| \frac{d x \mid}{d x t} \mid \leq \frac{11}{d x}
$$

cor 
i..e.

$$
\lambda^{2}=\frac{a \pm|u|}{a}<\frac{11}{a^{2}}
$$

$$
\alpha \leq \frac{1}{a \pm|| u \mid}
$$

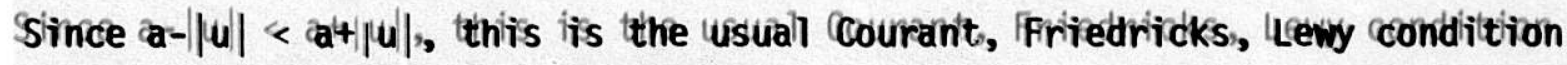
and is the same as conditition (13.110)).

In accordance with the Hirt alpproach, we now look for a second stabilititey critterion by rewritting Eqqs. ((3.42)) and ((3.43)). This its accompliished by expressing $H_{t t}$ and $u_{t t}$ in teems cof partial derivatives in $x$ lby lusing the coriginall diffiferentitial equations. Thus

$$
\begin{aligned}
& H_{t t t}=u_{t} H_{x} / x=u_{H} / x t t=a^{2} / / g u_{x} / t \\
& =\left(\left(u^{2}+a^{2}\right)\right) H_{x x}+\frac{2 a^{2} u}{g} u_{x x}+\ldots . \\
& u_{t t t}=-u_{x} u_{t}-u_{u_{x t}}=g H_{x t}=\frac{f u u_{x}}{t} \\
& =2 u \operatorname{ugh}_{x x}+\left(\left(u^{2}+a^{2}\right)\right) u_{x x}+\ldots .
\end{aligned}
$$

When these expressions are used in ((3.42) and ((3.43) we obttain the frollinowing expressions.

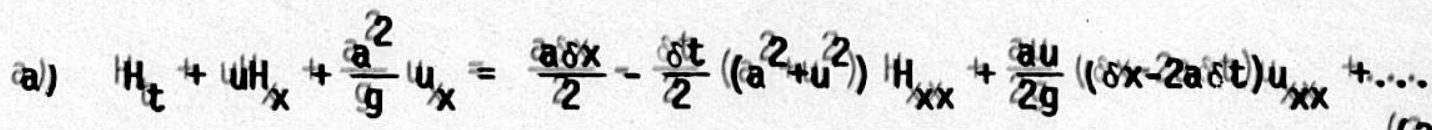

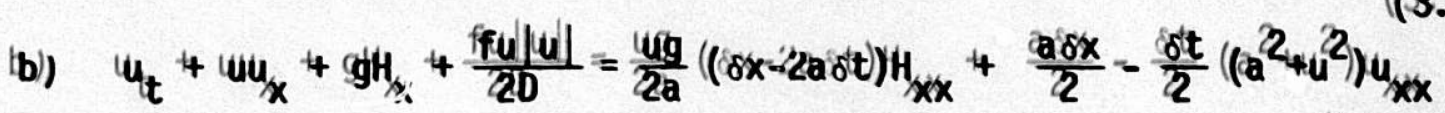

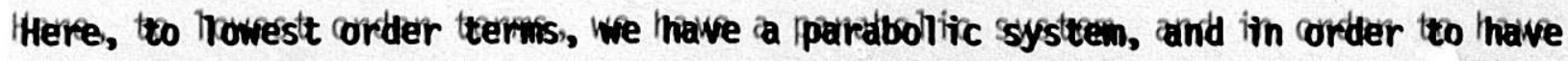

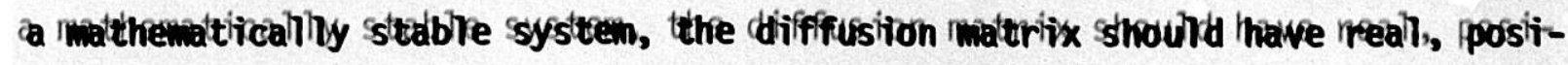
toive specturum. In the mottation off ((3.36)), we thave

$$
\begin{aligned}
& \left.D_{111}=D_{222}=\frac{\delta x}{22}\left(\left(a-c_{\alpha}\left(a^{2}+u^{2}\right)^{2}\right)\right)\right),
\end{aligned}
$$

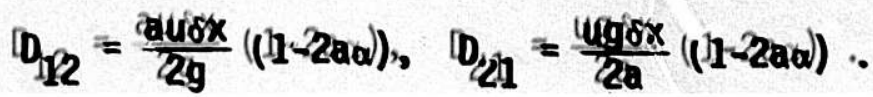


We firist obsenve that the spectrum in always real, since

$$
\left(\left(D_{111}-D_{222}\right)\right)^{2}+4 D_{12} D_{211}=u^{2} \delta x^{2}\left(11-2 a a_{i}\right)^{3}>0 .
$$

Referring to Eq. ((3.36c)) we cobserve

$$
\begin{aligned}
D_{111} D_{22}-D_{122} D_{21} & =\frac{\delta x^{2}}{4}\left[\left(\left(a-d\left(a^{2}+u^{2}\right)\right)\right)^{2}-u^{2}\left((1-2 a u)^{2}\right]\right] \\
& =\frac{\delta x^{2}}{4}\left(\left(a^{2}-u^{2}\right)\right)[[1-\alpha(a+u)][[1-\alpha(a-u))] \geq 0 .
\end{aligned}
$$

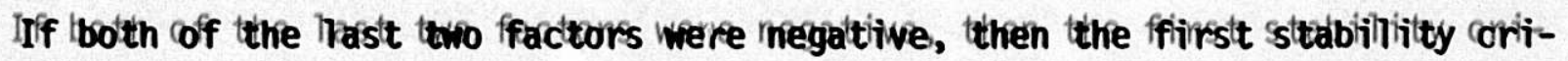

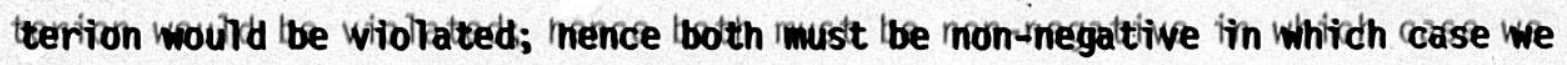
cobtain the fiorst sstabilitity coritterion.

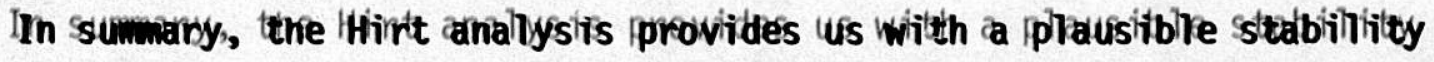

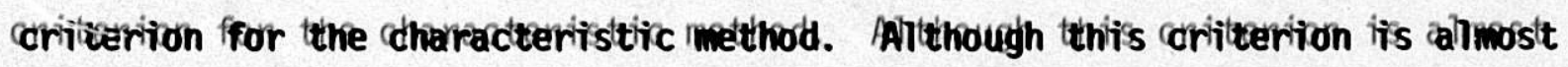

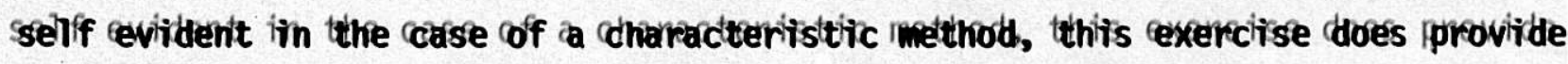
the conffidence and teechniquie for uise con cother approximationis. 


\section{LLAX METHOD}

The LLax ffiniite ddifference method ((cf.. LLax [[6], Rroache [[7]], Rottter [[8])) its a single sttep, explicicit method which its appliticable to the watter thamer equationis.

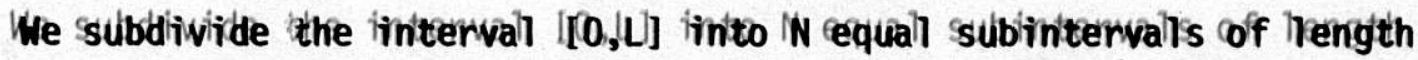
$\delta x=L / / N$ with the rmesh $x_{i j}=((i-1)) \delta x$ for $1 \leq$ i $i \leq 1 N+1$. Agalin, we iidentifify

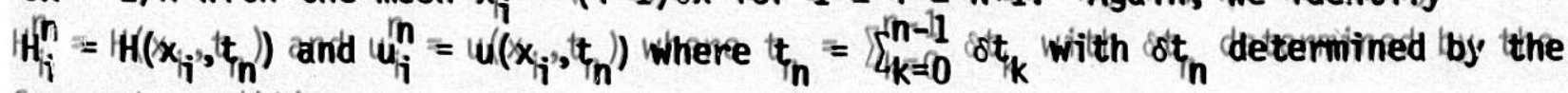
couran't coonditition

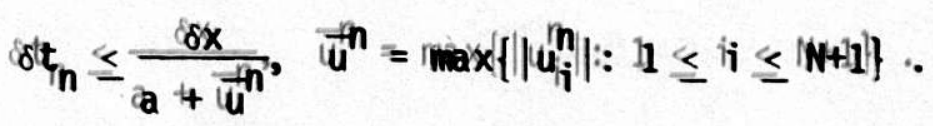

In practitice we selrect the equallitity stign.

\section{Diffiference Equationis}

The Laax diffiference method uses centrall spatival diffiferences and simgle forwartd timme diffiference but with a spattiall average replacing the piwot wahue.

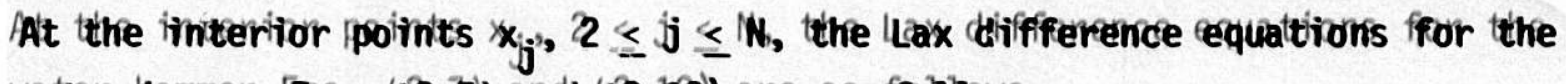
watter thaminer [Eqs. (22.7)) and (12.110) aree ais frothows.

$$
\begin{aligned}
& \text { (a) } \quad H_{j j}^{n+11}=\frac{1}{2}\left(H_{j-11}^{n}+H_{j+1}^{n}\right)=\frac{a}{2}\left[\left[u_{j j}^{n}\left(H_{j+1+1}^{n}-H_{j-1}^{n}\right)+\frac{a^{2}}{g}\left(\left(u_{j+11}^{n}-u_{j-11}^{n}\right)\right]\right.\right. \text {, }
\end{aligned}
$$

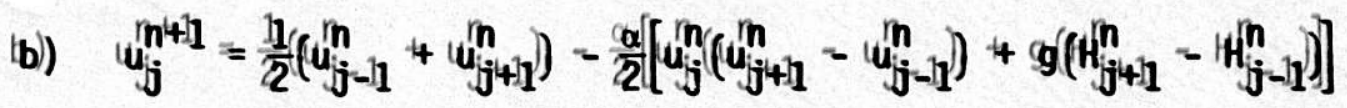

$$
\begin{aligned}
& =u_{j}^{m}\left|u_{j j}^{n}\right| f / / 20
\end{aligned}
$$

Where, as lustall, $\alpha=\delta$ st $/ \delta \%$.

\section{Boundary contititions}

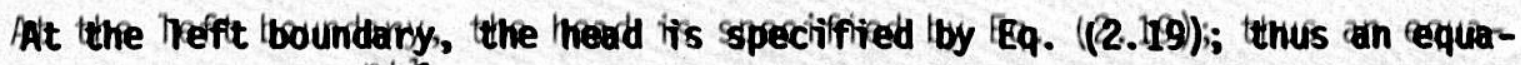

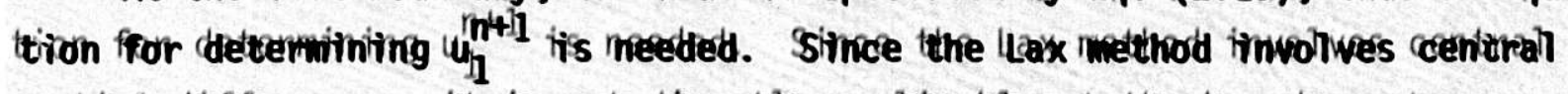

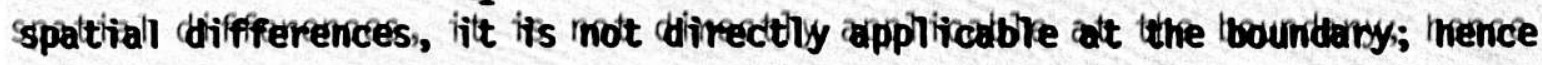

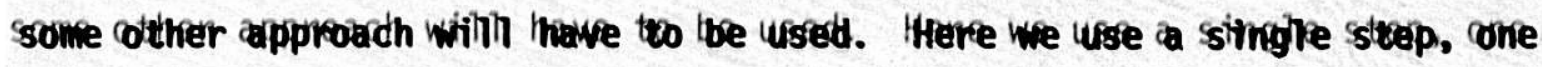

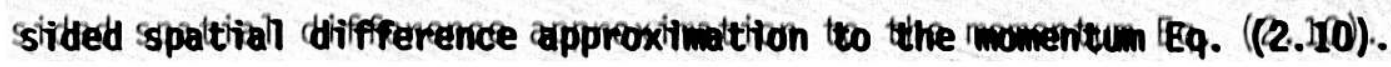




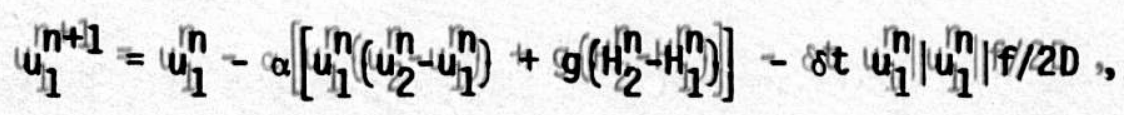

trogether with the boundany condiition ((2.119))

$$
H_{1}^{n+1}=H_{O R}
$$

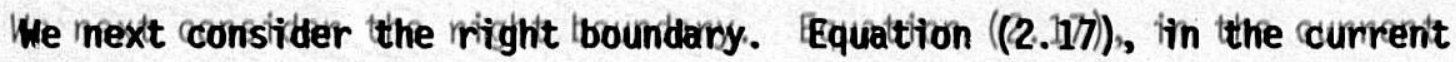
motalition, thas the form

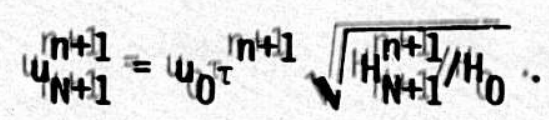

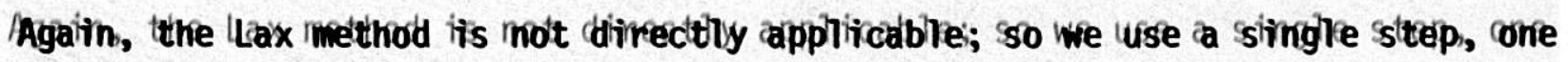
siided diffirerence capproximation to the momentum Eq. ((2.110))

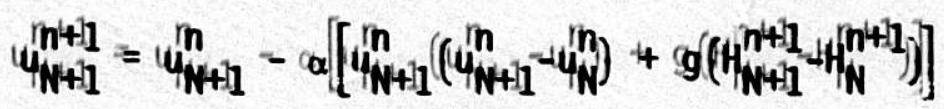

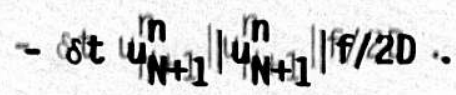

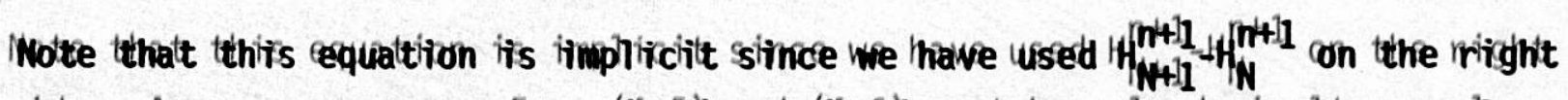

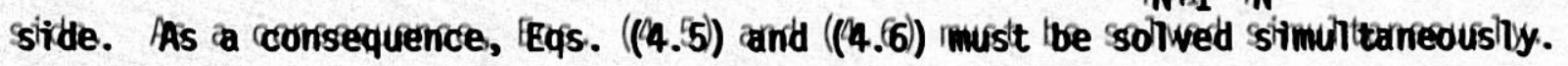

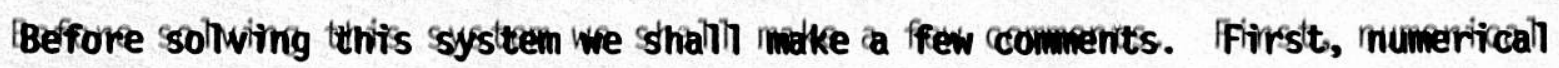
cexperizence thas convinced us that an expliiciit procedture alt this boundany with

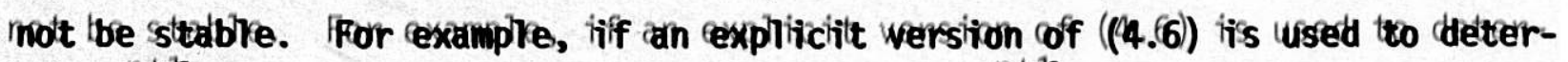

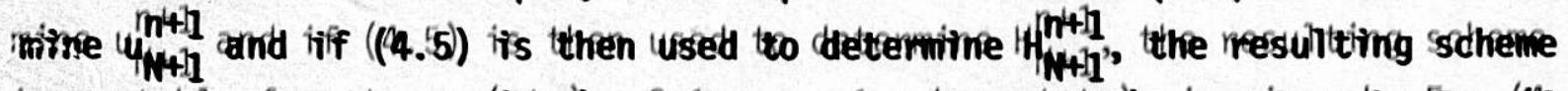

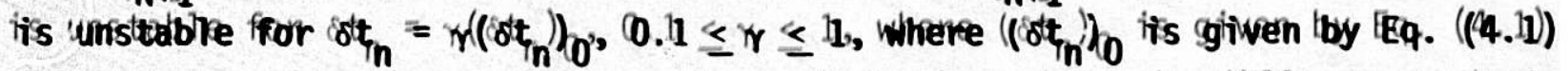
witth the equaliitey ssizgn. The ssecont remank its thatt simce the dififference sscheme

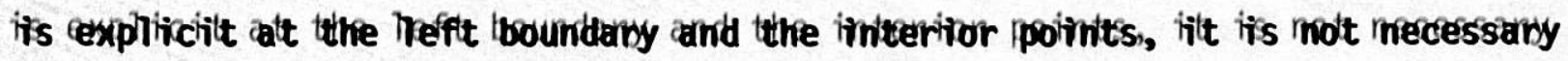

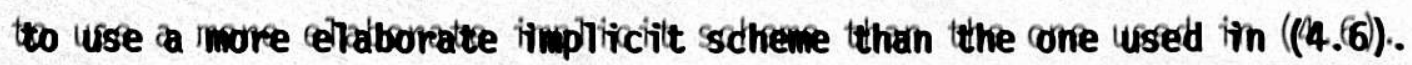

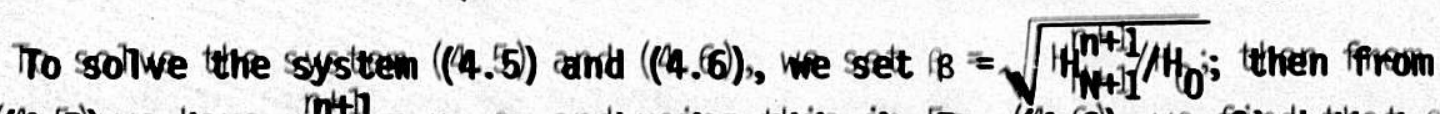

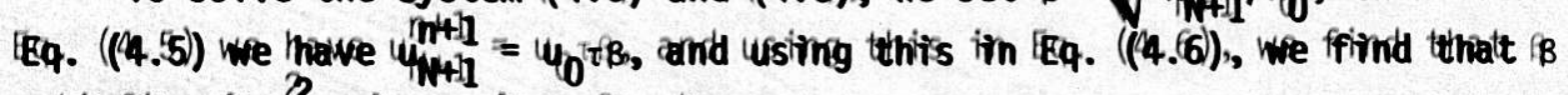

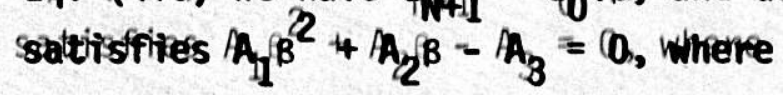
a) $A_{1}=0 x g t_{0}$
b) $\quad A_{2}=40^{2}$

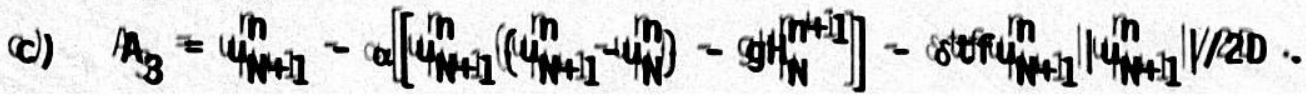


Sirnce $\beta$ its posititive, we select the posiitive root

$$
\beta=\left[\left[-u_{0} \tau+\sqrt{\left(u_{0} \tau\right)^{2}+4 a g H_{0} A_{3}}\right] / / 20.9 H_{0},\right.
$$

and

$$
\begin{aligned}
& \text { (a) } H_{N+1}^{n+11}=H_{0} B^{2} \\
& \text { b) } u_{N+1}^{n+11}=u_{0} t B .
\end{aligned}
$$

\section{Trunctition Eerror and Stabilliity}

Expanding Eqs. ((4.2) in a Taylior sseriies, and rettainimg the lowest corder termis in the truncation error, we cobtain

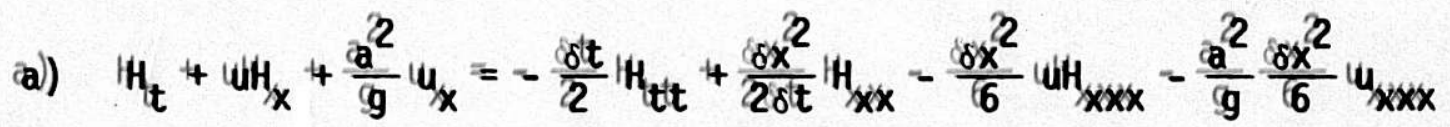

$$
\begin{aligned}
& +C\left(\left(8 t^{2}, 8 x^{4} / 18 t^{2}, 80 x^{3}\right)\right) \\
& \text { b) } u_{t}+u u_{x}+g g H_{x}+\frac{f f u|| u \mid}{2 D}=-\frac{\delta t}{2} u_{t t t}+\frac{\delta x^{2}}{2 \delta t t} u_{x x x}-\frac{\delta x^{2}}{6} u_{u} u_{x x x} \\
& \left.-\frac{\delta x^{2}}{6} g g H_{x x x x}+\cos \left(80 t^{2}, \delta x^{4} / / 80 t^{2}, 8 x^{3}\right)\right) \text {. }
\end{aligned}
$$

Assuming that we can ineglect the tremis inwolwing thived corder derivatives, we ffind that we thave a lpair of ssecond corder hypperbolitic equations which are mott

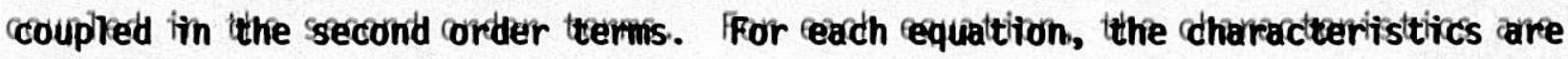
defefined tby

$$
\frac{d d x}{d t}= \pm \frac{\delta x}{d t}
$$

thence the domain of ionfluuence of the diffiference equation its the salue afs the

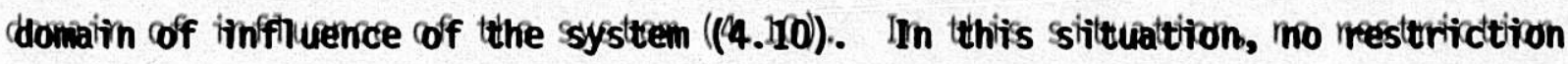

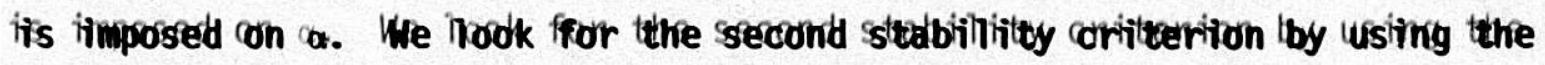

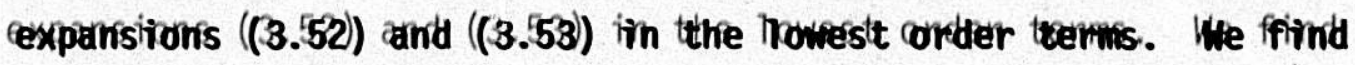

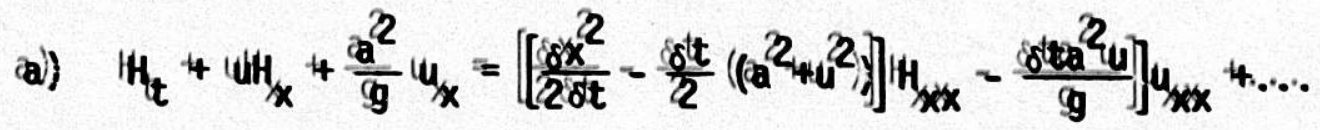

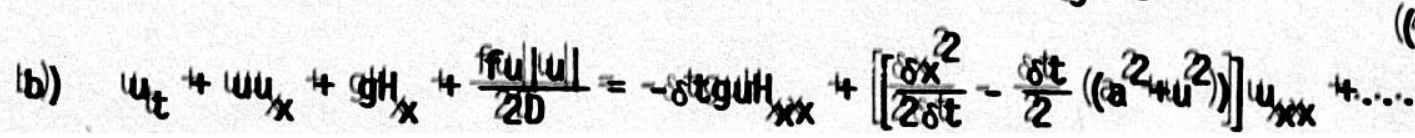


Thus we thave the frollhowing diffifusion mattrix coeffificients

$$
\begin{aligned}
& D_{111}=D_{22}=\frac{\delta t}{2}\left[\left[\frac{1}{2}-\left(\left(a^{2}+u^{2}\right)\right)\right],\right. \\
& D_{12}=-\frac{\delta a^{2} u}{g}, D_{21}=-\delta t \operatorname{tgu} .
\end{aligned}
$$

Referring too the coritteria in $((3.36))$, we cobsenve that ssince $D_{12}$ and $D_{211}$ thave

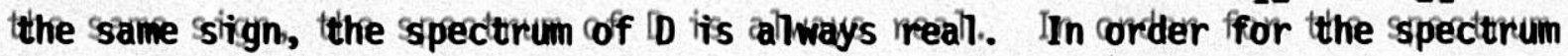
to the mon-megattive, we mustt thave

$$
D_{111} D_{222}-D_{122} D_{211}=\frac{\delta t^{2}}{4}\left[[ \frac { 1 1 } { \alpha ^ { 2 } } - ( ( a + u ) ) ^ { 2 } ] \left[\left[\frac{11}{\alpha^{2}}-\left((a-u)^{2}\right] \geq 0,\right.\right.\right.
$$

ii.e. the usuall couranit conditition

$$
a \leq \frac{1}{a+|| u \mid}
$$

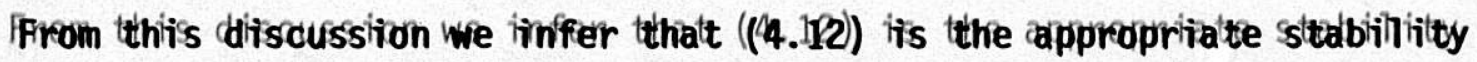
coritterion. Iof course, thiis dirscussion thas meglectted all thoundany effifectss; however, mumericall experizence has shown that iff the rrizght thand tboundany its

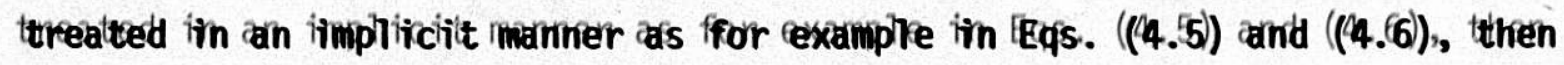

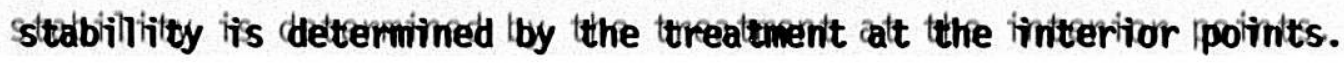




\section{GALERKIN METHOD WITH B-SPLINES}

When the lititeralture on computitationall methods in compressible lhydrodynamics is cexamined, the covemwherming tendency iss to uise the method of characteriistitics or ffinitte diffiference methods. However, ffinitte diffiference methods involive some diffificultities in the sattisffaction of boundany condititions.

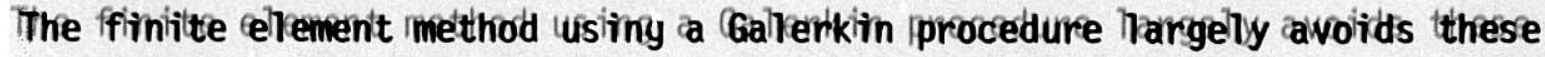
diffificulitives, and therefore offifer a potentitialiny weny atteractive alliternaltive.

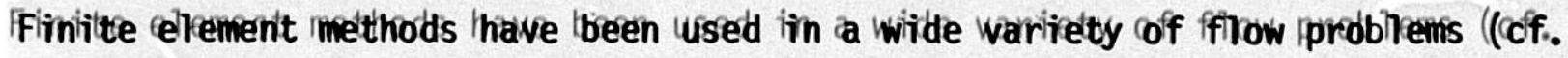
ref. [[9]]), but to the best off cour hnowledge, these method's thave mot been implemented for the ssolution of watterhamer transiients. IIn the ipresent appliicattion, we have used a Gallerkin procedure with an approximatting ssubspace

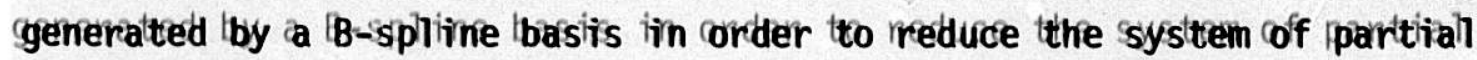
dififferentitiall equaltions to a sysittem of cordinarny dififerentitiall equations [[110].

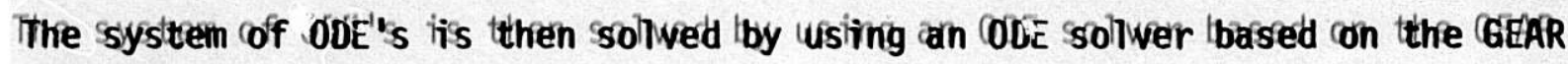
coode [[1]1]].

\section{Method of llimes}

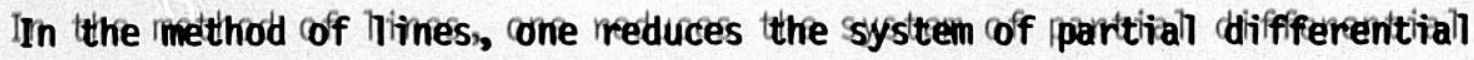
equatitions to a systtem of cordinary diffiferentivall equaltions. Leet

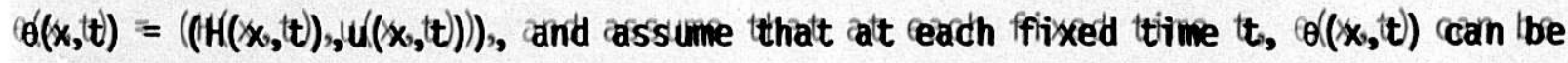

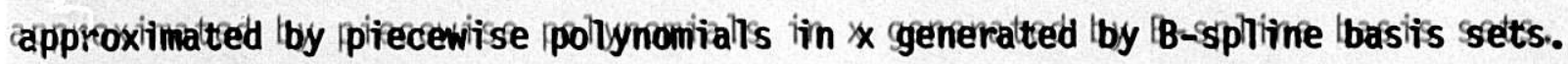

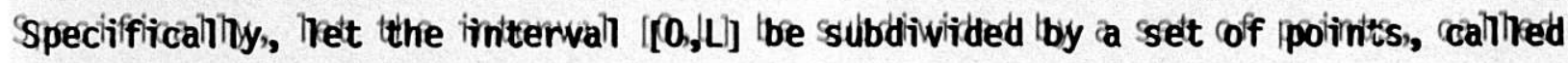

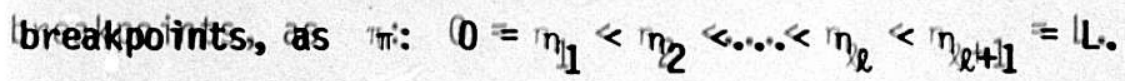

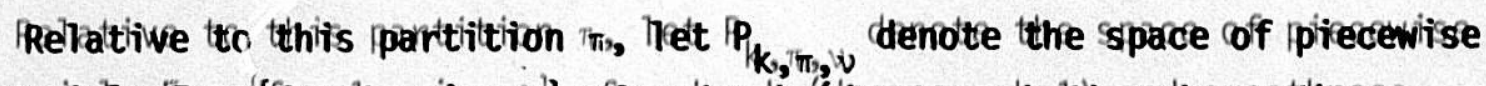
polinynomialiss if $=\left\{\left\{f_{i j}: 11 \leq i \leq \leq l\right\}\right\}$ cof corder $k k$ ((degree $\left.=\mid k-11\right)$ and smoothness

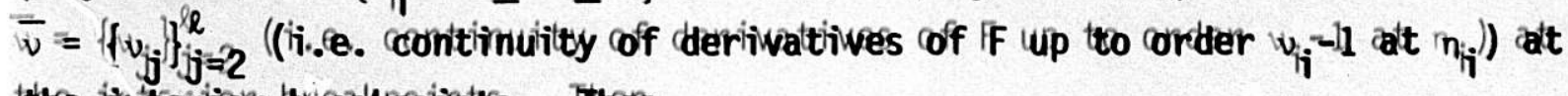
the inteiritor breakpointts. Then

$$
\operatorname{dim} \mathbb{P}_{k, j \pi, v}=m=k l=\sum_{i=2}^{l} w_{i j},
$$

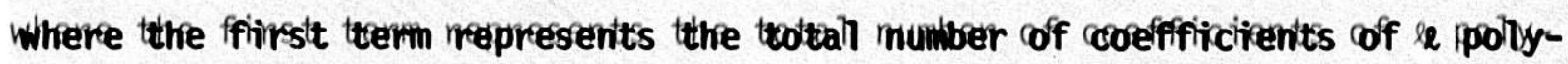

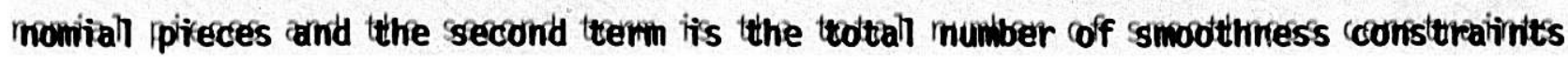


imposed alt the initerior breakpoints. We then seek an approximalte ssolution cof the form

$$
\theta\left(\left(x_{0}, t\right)\right)=\sum_{i=11}^{m} \Omega_{i j}((t)) w_{i j}((x))
$$

Where $\Omega_{i j}((t))=\left(\left(H_{i j}{ }^{\prime}(t)\right), u_{i j}((t))\right)^{\prime \prime}$ are unknown ttime dependent cooefficicients, and

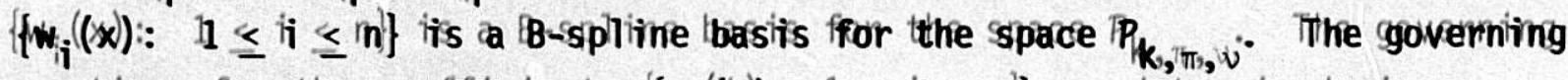
equattions fror the coeffificitients $\left\{\left(\Omega_{i j}((t)): 11 \leq i \leq n\right\}\right.$ are idettermined wita a

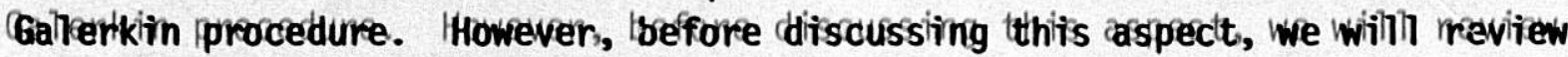
some importtant ffeatures of $B$-swilines.

\section{B-Spplinnes lBarsiss}

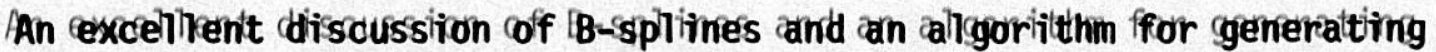

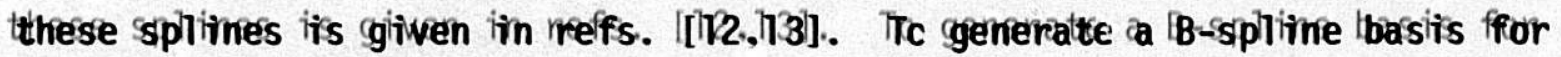

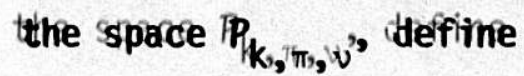

$$
q_{k}((s ; n))_{+4}^{\mid k-11}=\left\{\begin{array}{ll}
((s-n))^{\mid k-1} & s \geq n \\
0 & s<<n
\end{array},\right.
$$

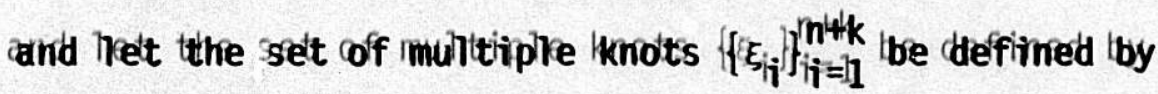

$$
\begin{aligned}
& \xi_{11}=\ldots .=\xi_{\xi_{k}}=n_{11}, \\
& \xi_{k+11}=\ldots .=\xi_{k+k+d}=1 / 2,2 \text {, } \\
& \because
\end{aligned}
$$

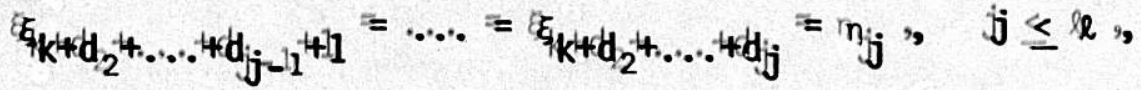

$$
\begin{aligned}
& \because \\
& \xi_{n+11}=\ldots . \xi_{n^{4+k}}=\eta_{l l^{+11}} .
\end{aligned}
$$

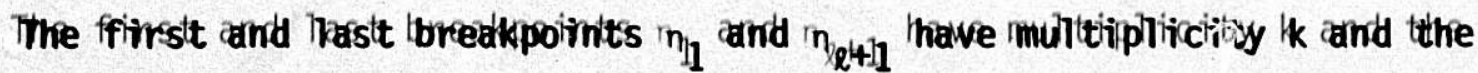

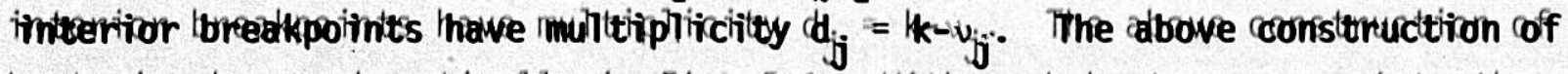

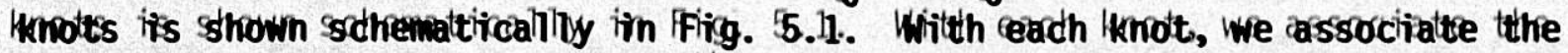

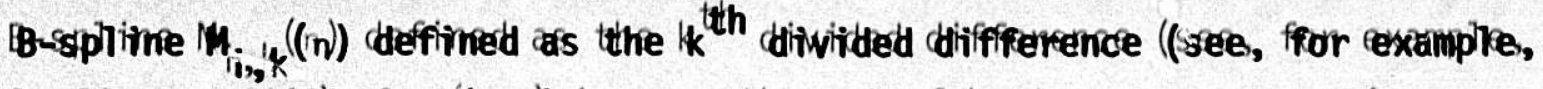

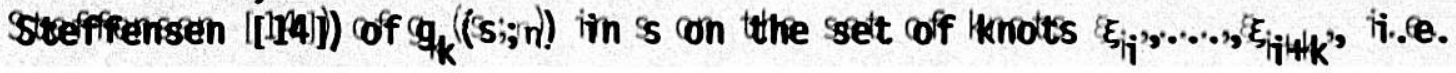




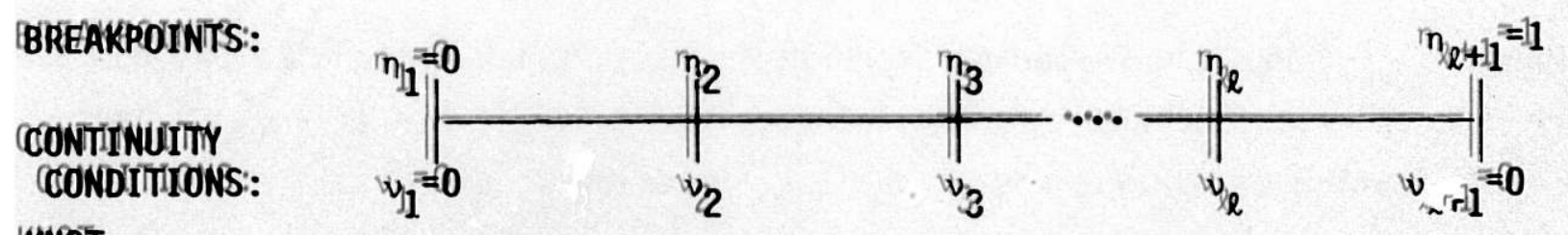

IKNOT

MULTIIPLICITIT:

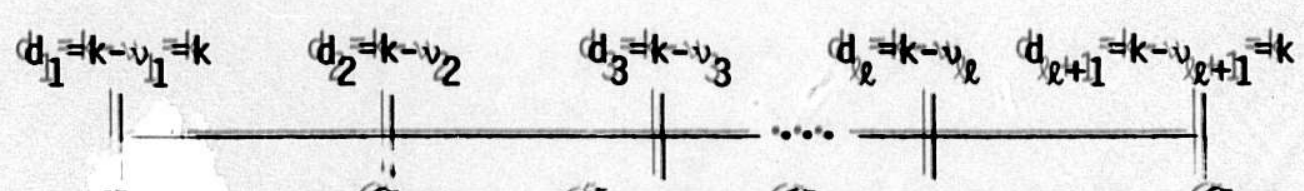

WNOTS:

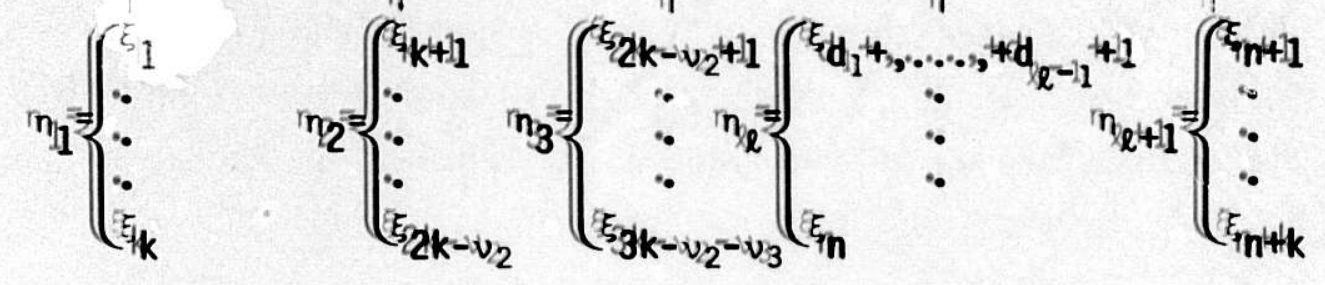

Fiig. 5.11 Schematitic diragram showing consteruction of throtts

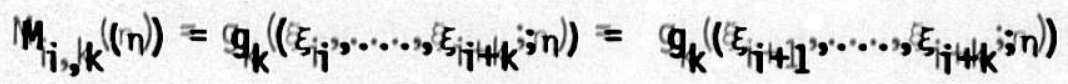

$$
\begin{aligned}
& =q_{k}\left(\xi_{j i}, \cdots, \cdots, \xi_{j i+1 k}-1 j^{;}, n\right) / /\left(\xi_{j i+k}-\xi_{j i}\right) \text {. }
\end{aligned}
$$

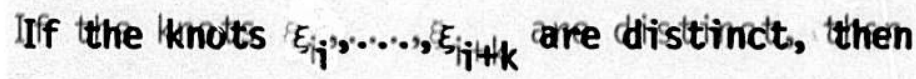

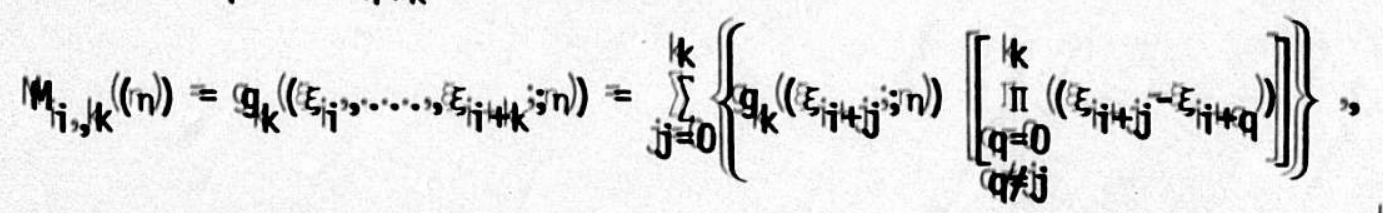

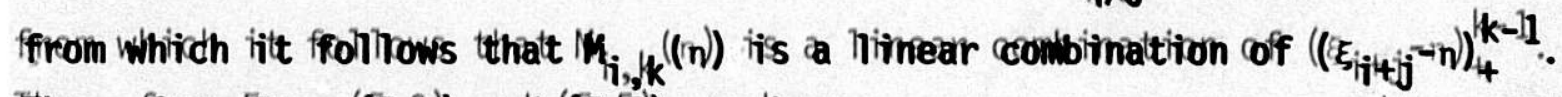
Thus, from EEqs. ((5.3) and ((5.5)), we thave

$$
M_{i, j k}((n))=0 \text { fror } n / E\left[\left[\xi_{i j}, \xi_{i j+t k}\right]\right. \text {, }
$$

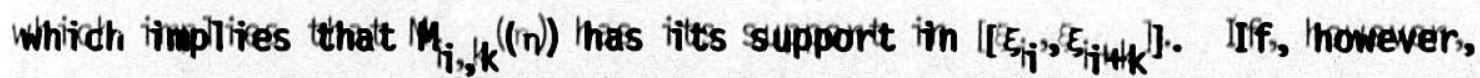

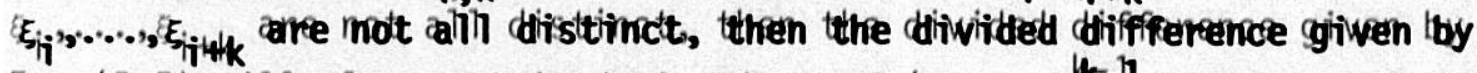

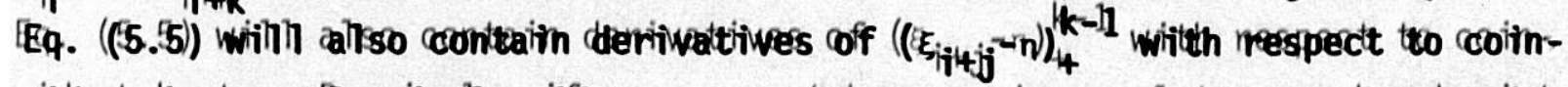

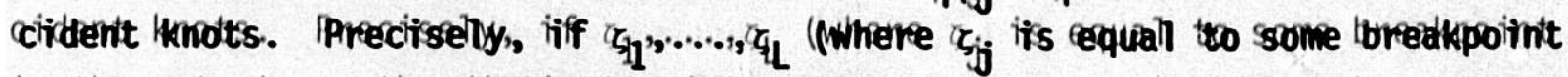

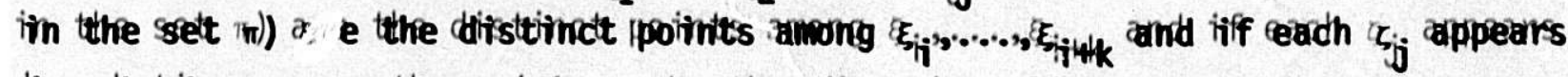

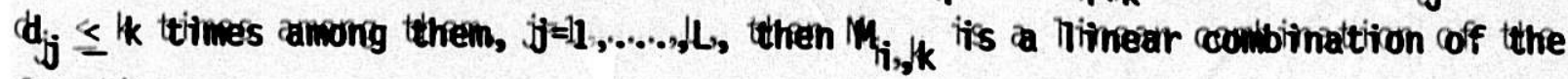
frunctionis

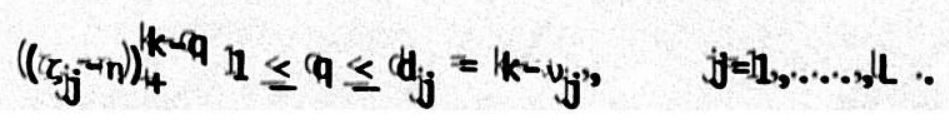




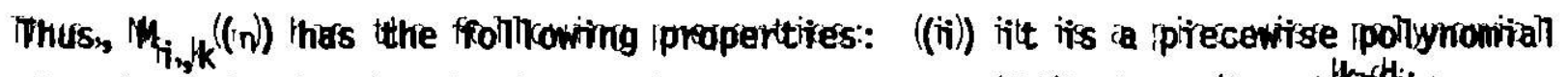

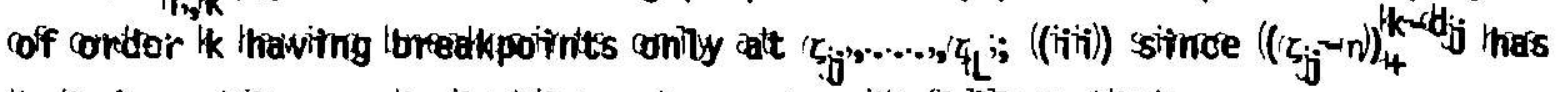

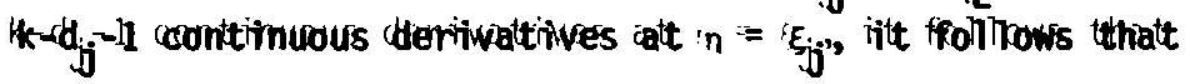

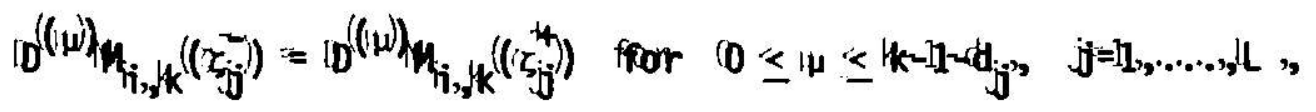

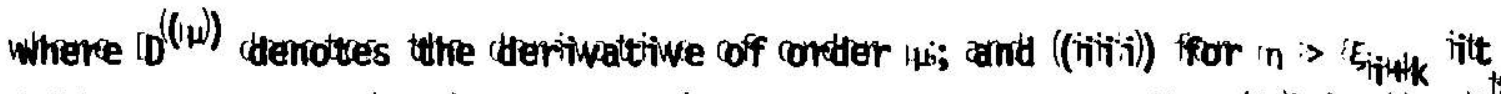

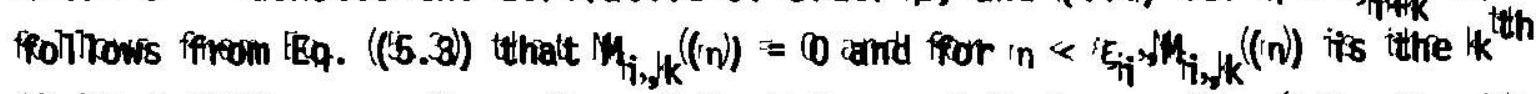

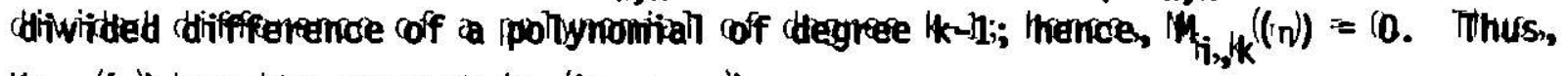
$M_{i, j k}((n))$ thas iitts ssupportt in $\left(\left(\xi_{j i}, \xi_{j i+4 k}\right)\right)$.

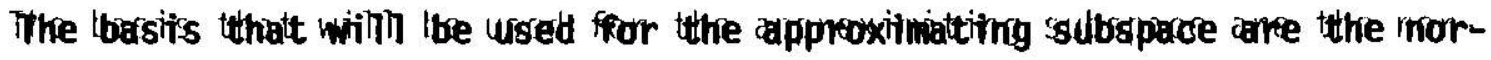
imalizzed lBusplitimes (deffirned lby

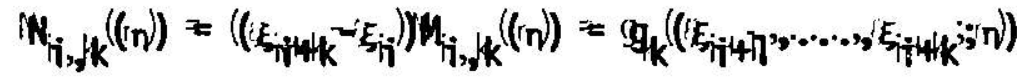

$$
\begin{aligned}
& =q_{k}\left(\left(\xi_{i j}, \ldots, \ldots, \xi_{j i}+k-11^{i n}\right)\right) \text {, }
\end{aligned}
$$

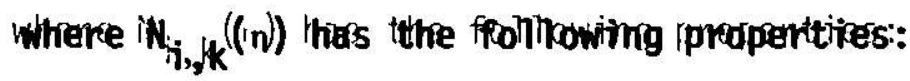

((ii)) momilitizattion proppentoy:

$$
\sum_{i=1}^{m} W_{i j, j k}((n)) \equiv 1 ; ;
$$

((iii)) convex recursition rpreaperty:

$$
\begin{aligned}
& N_{i, j, k}((n))=\frac{1 n-\xi_{i j}}{\xi_{i j+k-11^{2} \xi_{i j}}} M_{i j, j k-11}((n)) \\
& \left.+\frac{\left(\xi_{i j+k}-\right.\text { in }}{\left(\xi_{i j+k}-\left(\xi_{i j+1}\right.\right.} N_{i j+11,3 k-11}(v n)\right) \text {, ffor } \xi_{j i} \leq 1 n<\left(\xi_{j i+1 k}\right. \text {, }
\end{aligned}
$$

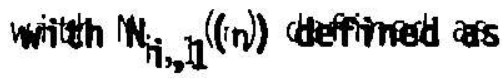

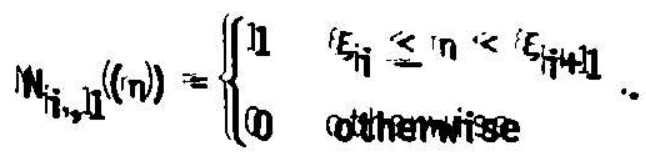

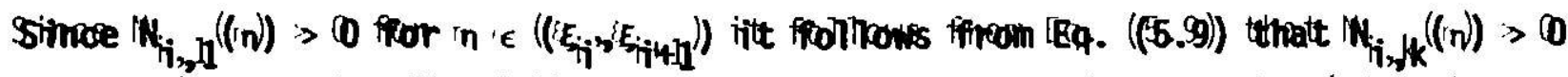

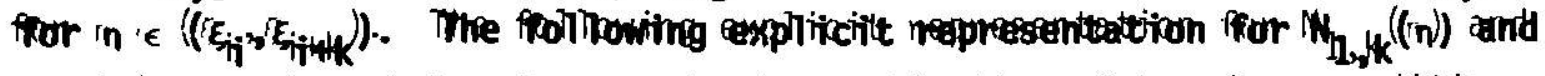

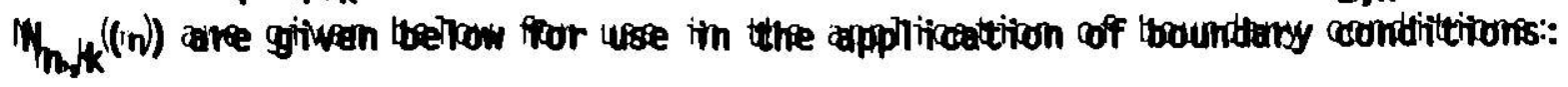




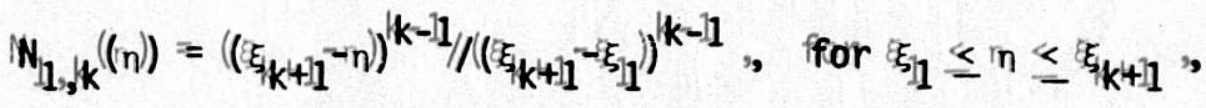

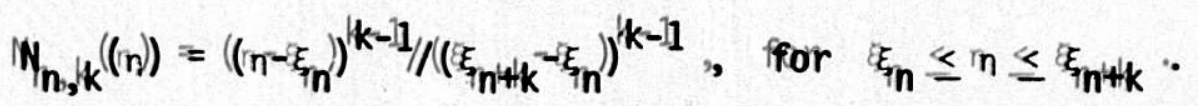

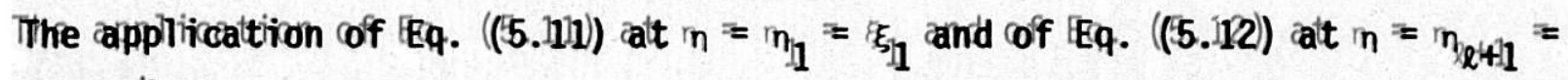
mntlk givives

$$
\begin{aligned}
& W_{1, j k}\left(\left(n_{11}\right)\right)=1, \quad W_{1,}^{\prime}, k\left(\left(n_{11}\right)\right)=-((k-11)) /\left(\left(m_{2}-n_{11}\right)\right), \\
& N_{n, j k}\left(\left(n_{l \ell+11}\right)\right)=11, \quad W_{n, j k}^{\prime}\left(\left(n_{l \ell+11}\right)\right)=((k-11)) /\left(\left(n_{l \ell+11}-n_{l \ell}\right)\right) .
\end{aligned}
$$

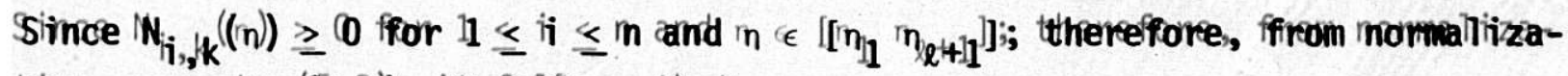
ttion properity $((5.8))$, iitt frolinows that

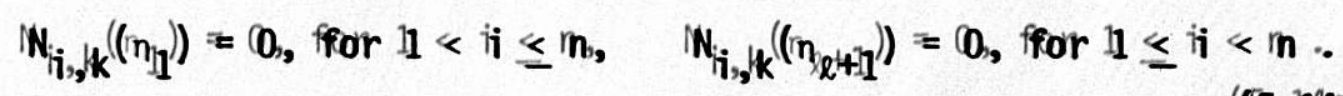

For the wallues of the derivattives of $N_{i, j, k}((n))$ alt the end breakpoinits, we

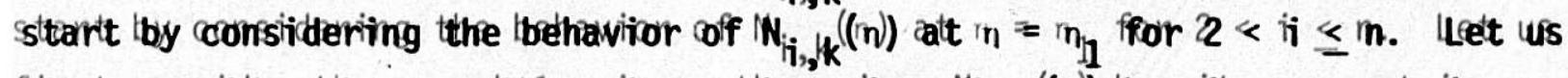

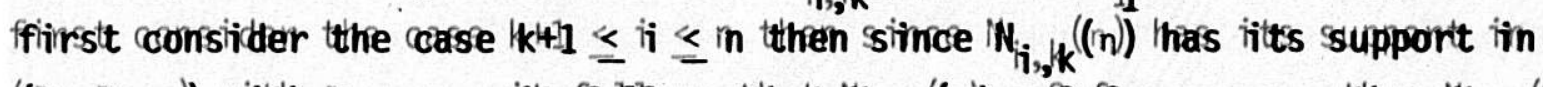

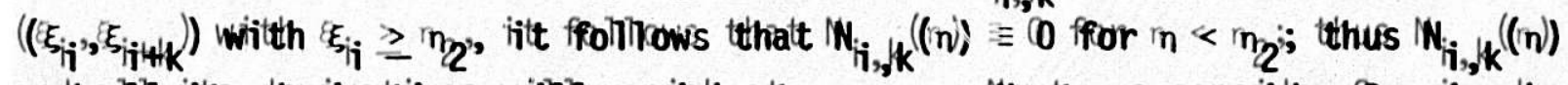

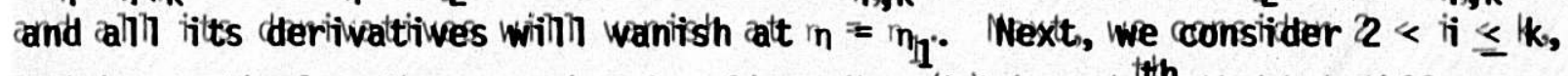

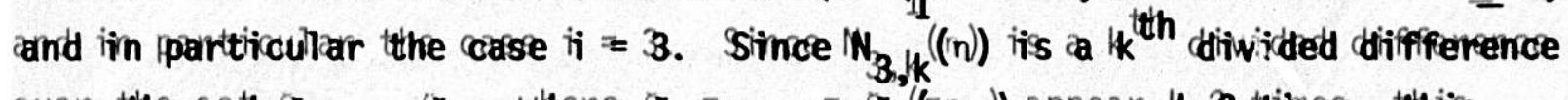

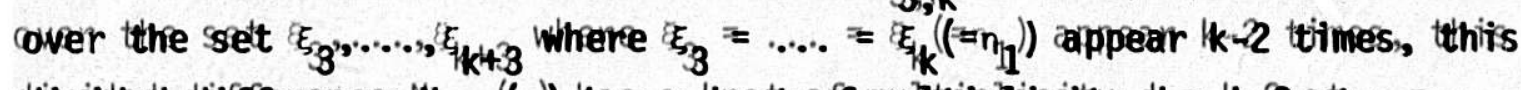

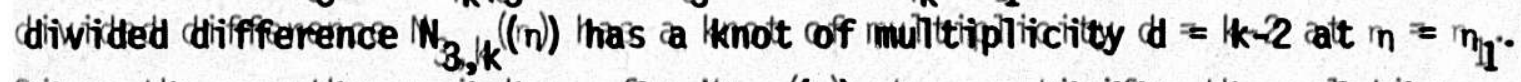

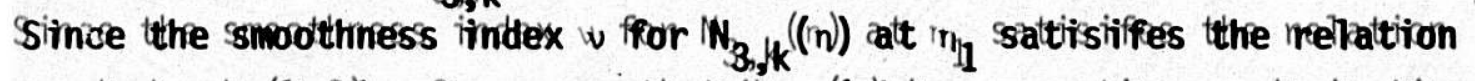

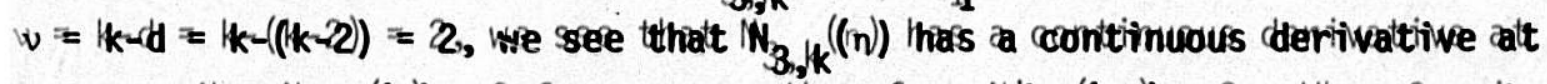

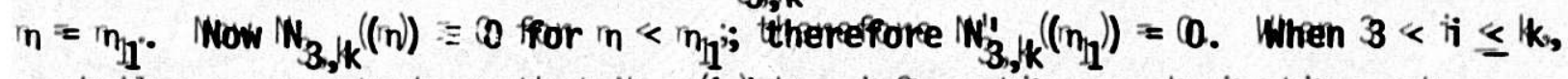

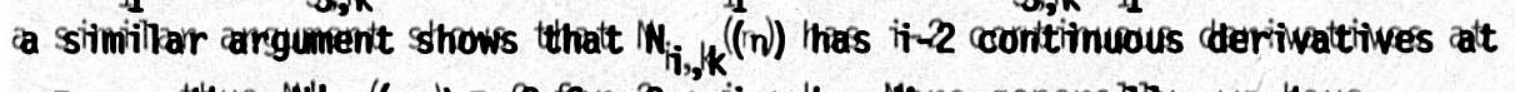

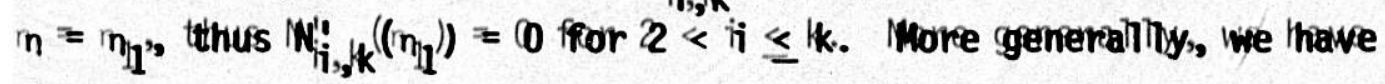

$$
\text { Nit, }((q))\left(\left(m_{11}\right)\right)=0, \text { for }(0 \leq q \leq i j-2 \text { and } 2 \leq i \leq k \text {. }
$$

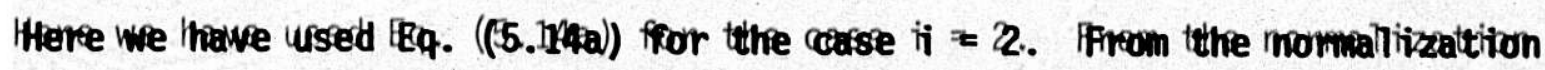
proppentoy (55.8)) we thave 


$$
\sum_{i=1}^{m} W_{i, j, k}((n)) \equiv 0, \text { for } n \in\left[\left[n_{11}, n_{l+1}\right]\right] \text {. }
$$

The use of Eq. ((5.115) in Eq. ((5.116) gives wis

$$
w_{1, k}\left(\left(n_{11}\right)+w_{2, k}\left(\left(n_{11}\right)\right)=0\right.
$$

with $\left.W_{i, j}\left(n_{1}\right)\right)$ given by Eq. ( $(5.133 a)$. AAnallogous angumenit willi sshow that

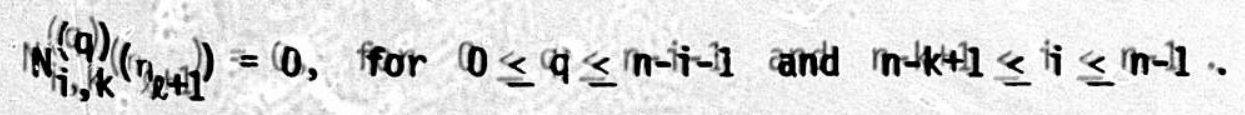

Thus, from EE. ((5.116)) and Eqq. ((5.188), we obtaiin

$$
W_{n-1, j, k}\left(\left(n_{l+11}\right)+W_{n, k}\left(\left(n_{l+11}\right)\right)=0\right.
$$

witth $W_{n, k}^{\prime}\left(i_{n+11}\right)$ given by Eq. ( $(5.1133 b)$.

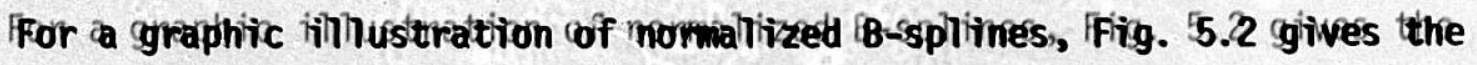

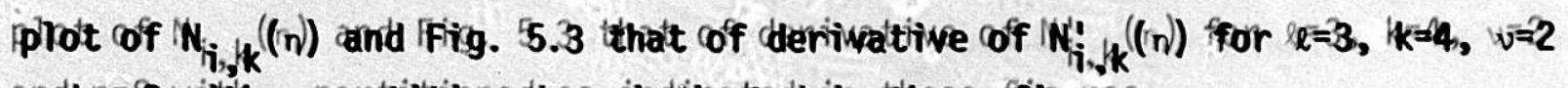
and $m=8$ with in ipartititioned as inddicatted in these firgures.

\section{Appoproxinate Equationis}

In tremis of nommalizzed B-splines, expatisiton (5.2) takes the from

$$
\theta((x, t))=\sum_{j=1}^{n} \Omega_{j j}((t)) W_{j, j, k}((x))
$$

where $\Omega_{j j}(t t)=\left(\left(H_{j j}(t t)\right), u_{j j}(t(t))\right)^{\prime}$.

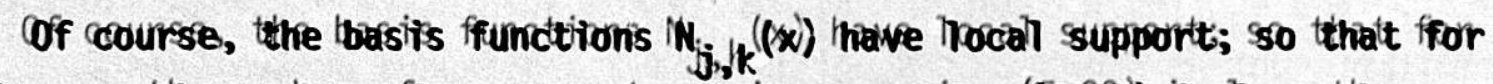

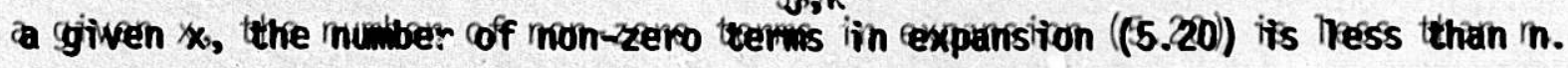

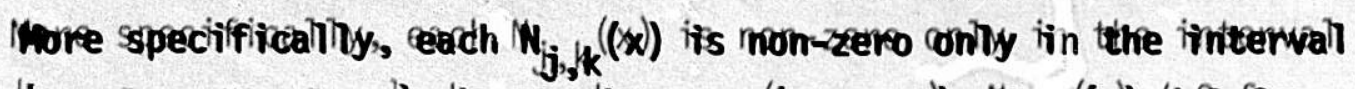

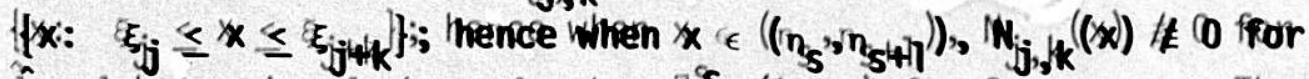

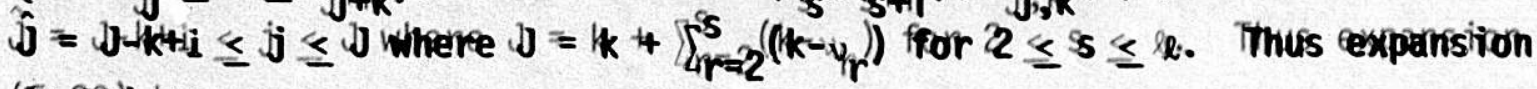
(55.20) tbecomes

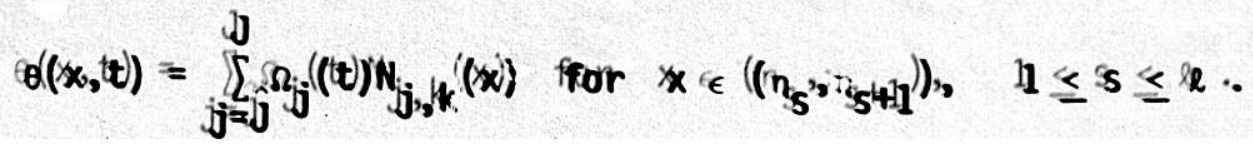



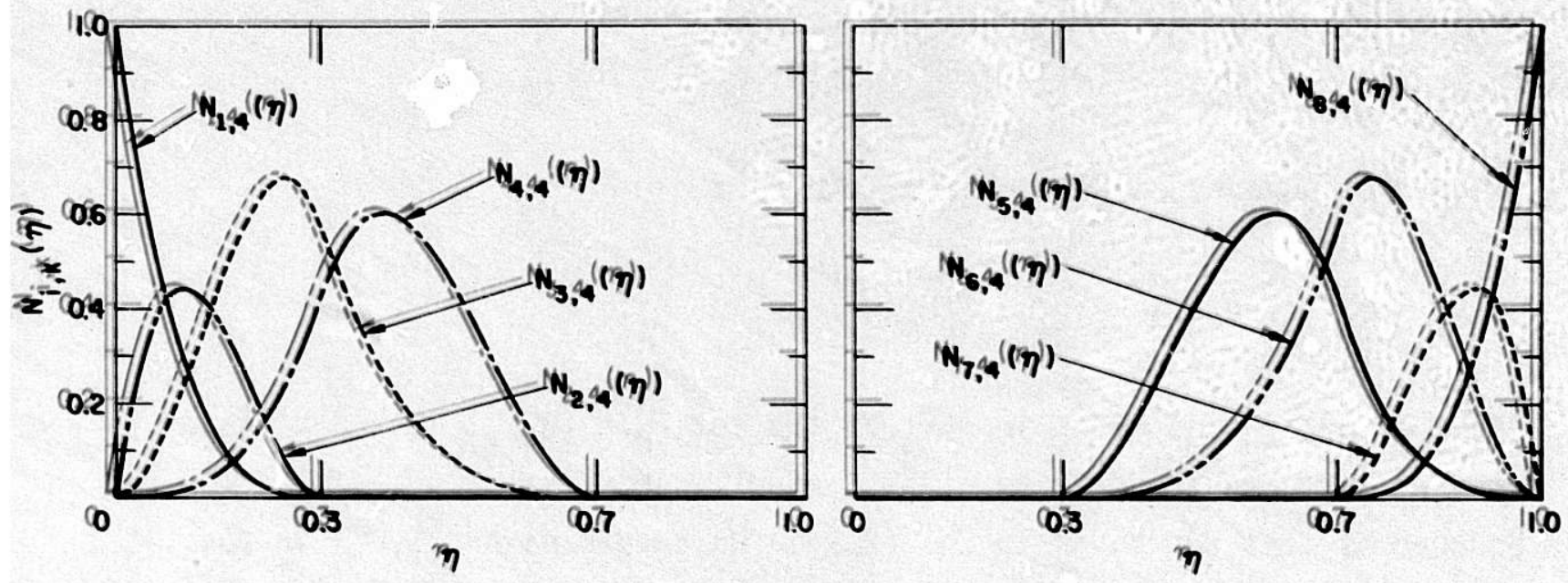

Fiig. 5.2 Caraphs of lB-splines $N_{i, k}(n)$ for $k=4, v=2$ and $l l=3$ with breakpointes at in $=20,0.3,0.77$ and 11.0
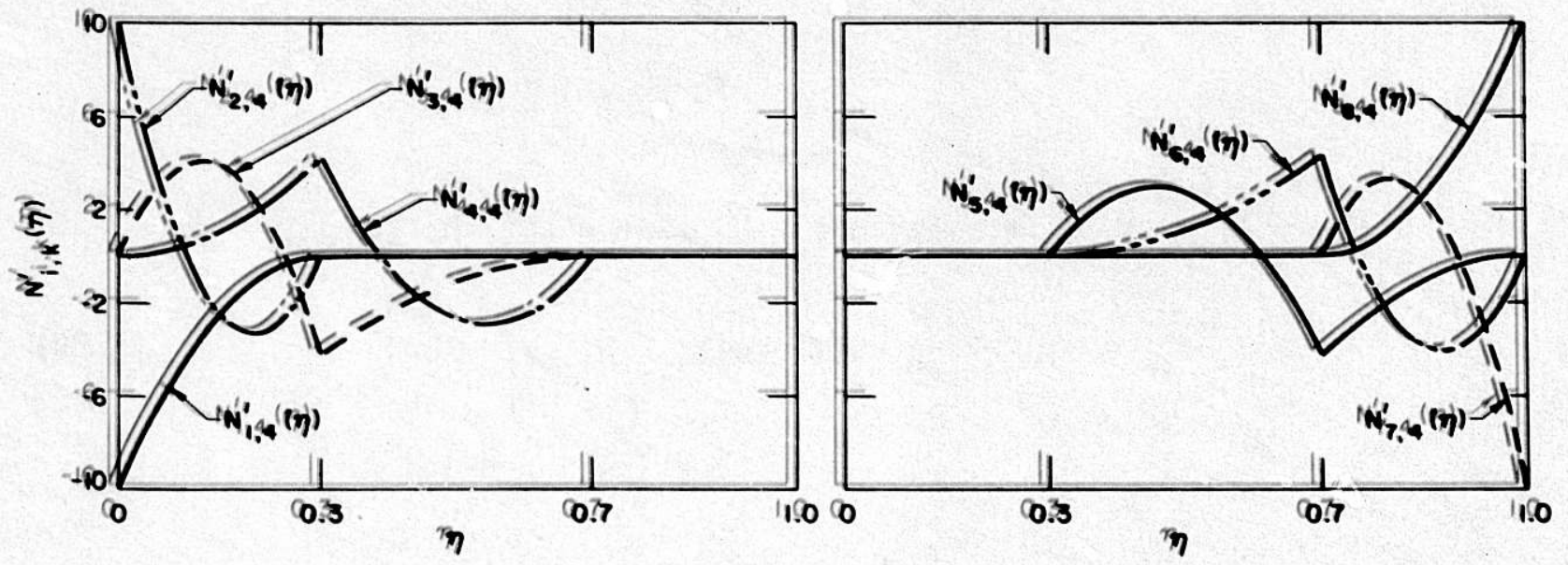

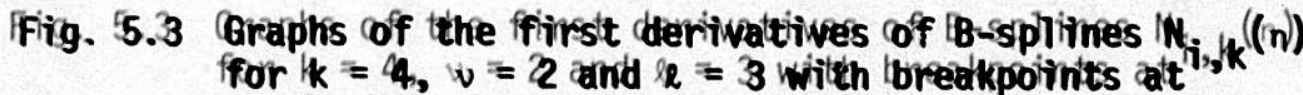
$n=0,0.3,0.7$ and 1.0 
Now, consivider the Gallerkin procedure for the systtem $((2.77)$ and $((2.10)$. Starting with the boundany conditition $((2.189))$ on the head, we ffind from conditions $((5.113)),(5.114)$ that

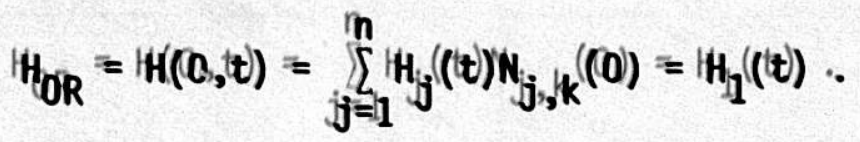

The riight looundany condition takes the form

$$
u_{n}((t))=\tau_{t}\left(t_{t}\right) u_{0} \sqrt{H_{n}((t)) / H_{b_{0}}} .
$$

These boundany condititions imply that the set $\pi_{i H}=\left\{N_{j, j, k}: 2 \leq j \leq m\right\}$ can be

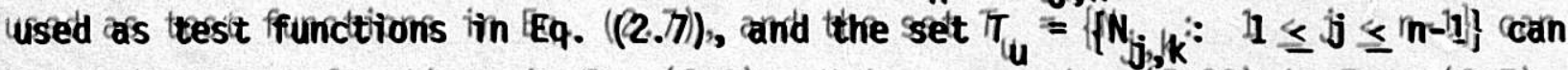

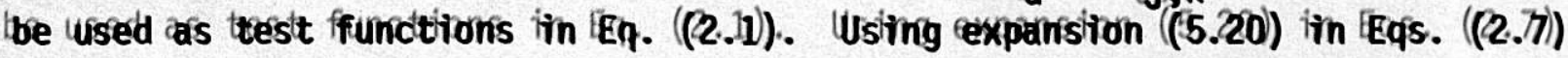

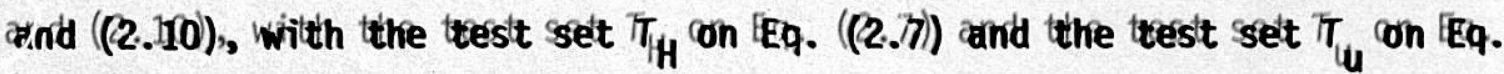
((2.110)), we thave the frolliowing syssteen.

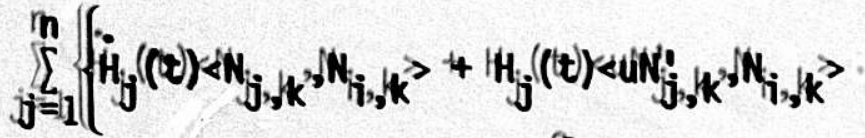

$$
\begin{aligned}
& \left.\left.+\frac{a^{2}}{g} u_{j}((t))<N_{j, j}, k^{,}, M_{i, j, k}\right)\right\}=0 \text {, } \\
& \text { for } 2 \leq \leq i \leq m \text {. }
\end{aligned}
$$

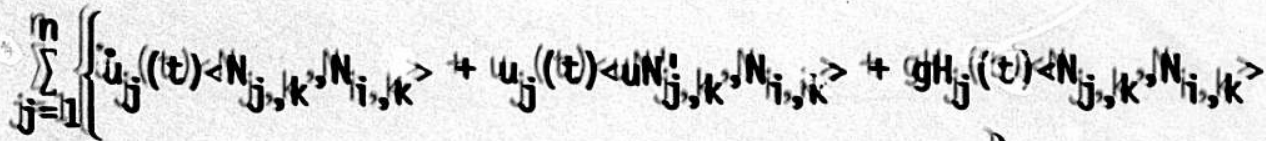

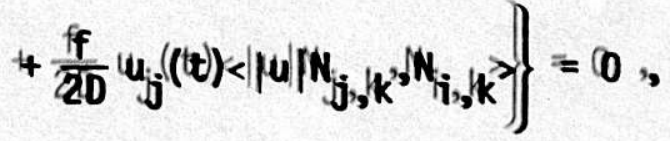

$$
\begin{aligned}
& \text { froo } 11 \leq i \leq m-11 \text {, }
\end{aligned}
$$

where $\left.\ddot{H}_{j j}((t))=\frac{d d}{d t} H_{i j}((t)), \dot{u}_{j j}(t t)\right)=\frac{d d}{d t} u_{i j j}((t))$, ant

$$
\langle w, w\rangle=\int_{0}^{l} w(x) w((x)) d x .
$$

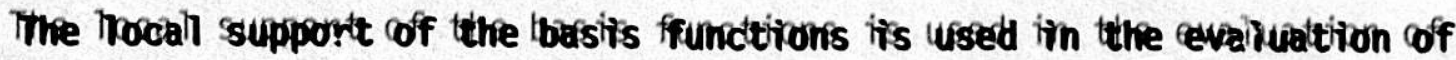

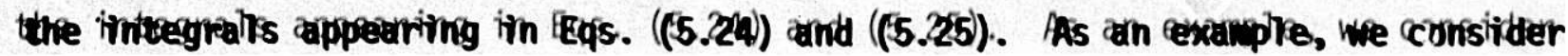




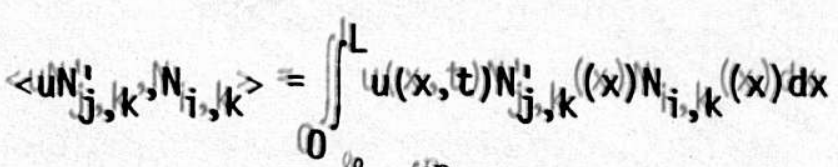

$$
\begin{aligned}
& \left.=\sum_{s=1}^{l} \int_{n_{s}}^{n_{s}+1} u(x, t)\right) N_{j}((x)) N_{i, j}((x)) d x .
\end{aligned}
$$

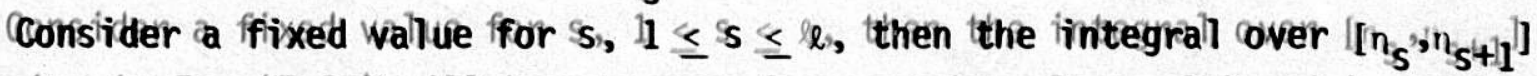
appearing in Eq. (5.27) will it inon-zero for certain wallues of $j \mathrm{j}$ and $i \mathrm{i}$. Specifficallny, sett $J=J(s))=k+\sum_{r=2}^{s}\left(\left(k-u_{r}\right)\right)$ and $\left.\hat{J}=\hat{J}(s)\right)=J((s))-k+1$, , then $N_{j, j, k}((x)) N_{i, j, k}((x)) \neq 0$ in $\left(\left(n_{s}, n_{s+1+1}\right)\right)$ conlly when $\left.\left.\hat{j}(s)\right) \leq i, j, j \leq J(s)\right)$. Each integral is evalluatted by Gauss-Legendre quadratture over $\left(\left(n_{s}, n_{s+1}\right)\right.$ so that

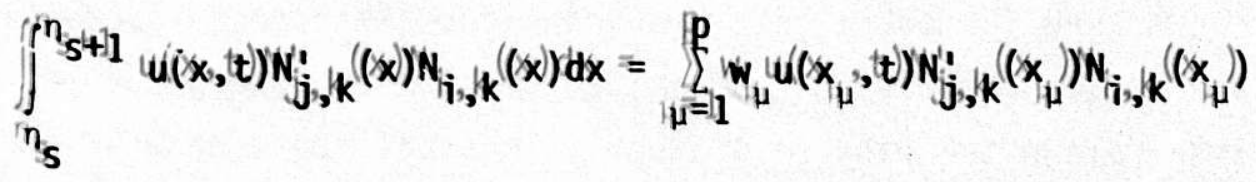

Where $\left\{\left[x_{\mu}, w_{\mu}\right\}\right.$ are the Gaussiaan points and weights relative to the interval

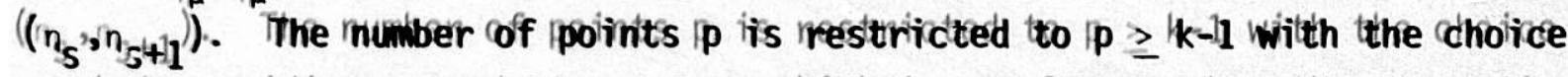
$|p=| k-1$ provitiong a quadratture error wivich is mot lrarger that the truncaltion

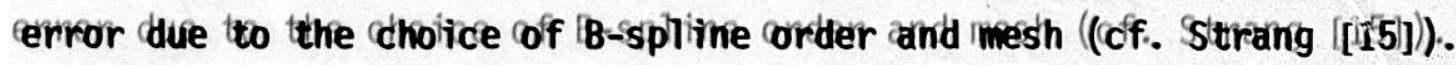

The equations ( $(5.222-5.25)$ form a systtem of 1 wixed alrgebratic-diffiferentitial equations of the follhowing form:
a) $\quad(0=g(y, z, t, t)$
(b) $\quad A \dot{a}=f(y, j z, t)$

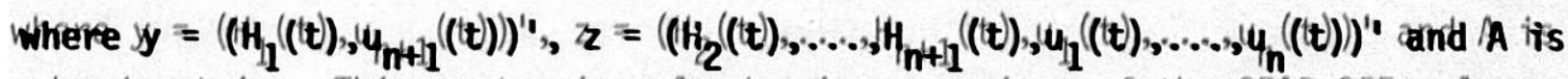

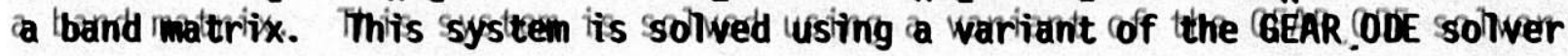
[1]11].

Some cobsenvations of Gallerkin teype approximattions are in corder. Firsit,

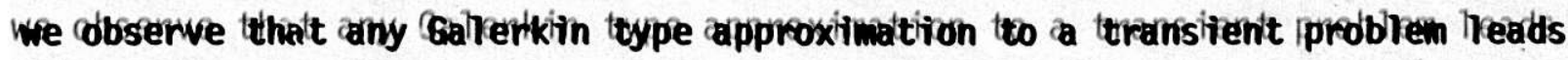

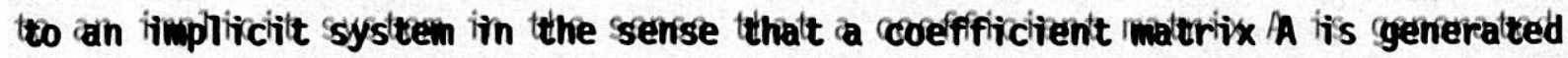

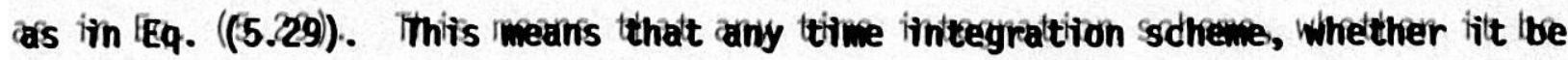

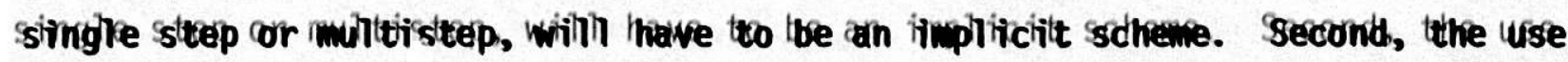

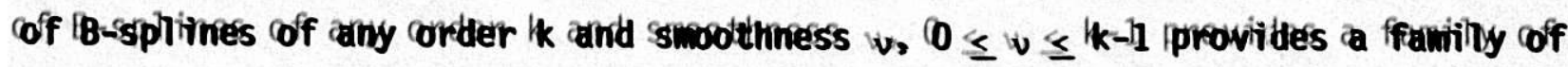

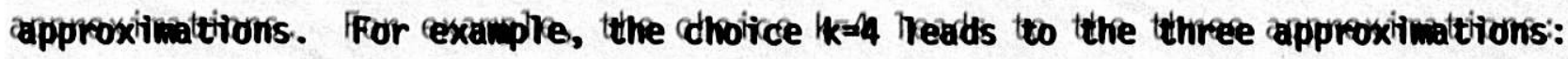
ssworth cubibics $((v=3))$, Hewitre cubibics $((v=2))$, and contimuous cubibics $((v=1))$. 


\section{COMPARISON OF LIINEAR AND QUUADRATIIC IINTERPOLATTION FOOR THE IMETHOD OF CHAARACTERISTIICS}

From section 2, we recall that there are two approximations in the method of characteristics. First, there is the approximation ((3.110) to the integral which generattes an asymptotic error of corder $\left(0\left(s t^{2}\right)\right)$. The second approximation involives the interpolation of the griid point wallues alt a given time wallue. We thave discuissed the use of liinear and quadratic interpolation in section 3. Here we will compare the cerrors cencountered with these schemes and we shally watter thamer problems corresponding to choices $m=1$ and 2 .

Before making the comparison, we know from experience for smalli densitity changes and att how welocitities that alt any given tiime, the pressure profilite is

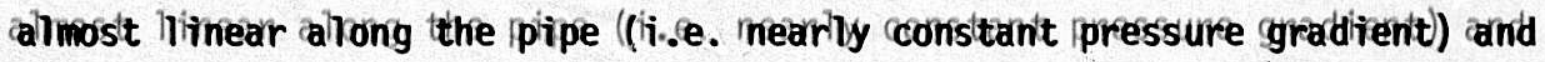

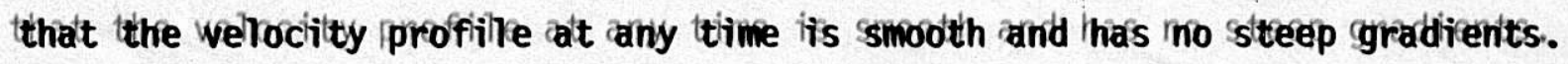
Thus, we expect good accuracy with a weny coarse spattiall mesh.

In Tables 6.11 and 6.2 , we present the dolata at three diffiferent points

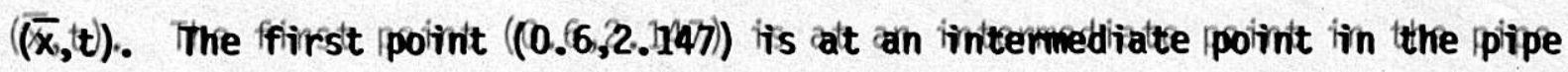

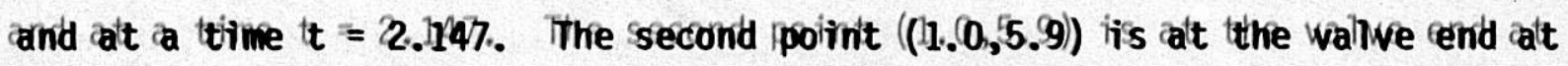
the time $t=5.9$ when the walive thas clrosed. The third point $((0.22,7.7728)$ its alt a point inear the reservoir end at the tiime when the fflow thas reversed. The benchmark wallues are taken as the quadratio interpolation witth $n=80$.

In Trable 6.11, we present the resultts for the case $m=11$. We firrsit cobsenve that in allh cases, the accuracy is weny good even when $I N=5$ where the

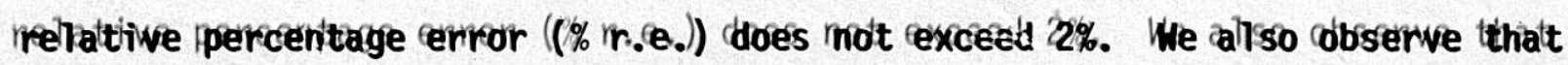

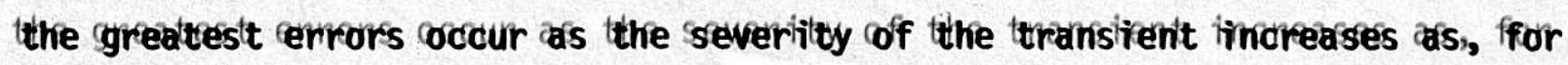
cexample, near $t=7.7728 \mathrm{sec}$. ffor the carse of $\mathrm{m}=22$ as compared to that of

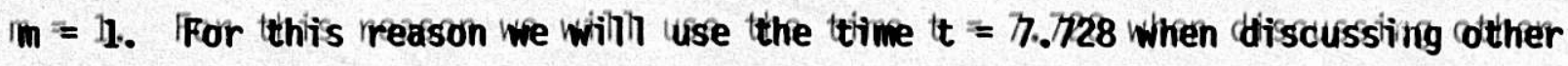
mumericall methods.

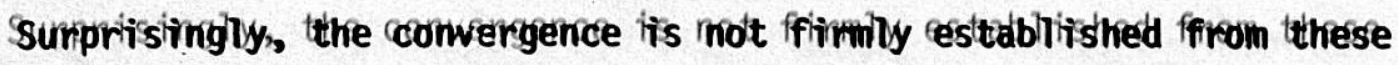
twables. Fror examples, consider the welocitities in Trable f6.1Tc and frour success ive diffiferences for $N=55,110, \ldots ., \ldots, 80$. Then we fimd:

\begin{tabular}{|c|c|c|c|c|}
\hline $\begin{array}{l}\text { Limear } \\
\text { Quaderatiotic }\end{array}$ & $\begin{array}{l}4.1776(-3) \\
2.4488(-33)\end{array}$ & $\begin{array}{l}0.234(-3) \\
0.11188(-33)\end{array}$ & $\begin{array}{l}0.447(-33) \\
0.252(-33)\end{array}$ & $\begin{array}{l}0.457(-3) \\
(0.2248(-3))\end{array}$ \\
\hline
\end{tabular}


TABLEE 6. Ia Pressure thead and Vellocity at $(\bar{x}, t)$

$N=\#$ variables, $\bar{x}=x / L=0.6, \quad t=2.1147 \mathrm{sec}, u_{0}=3.5 \mathrm{ft} / \mathrm{sec}, \tau=((1-t / / t c) \quad(\mathrm{m}=1 \mathrm{l})$

\begin{tabular}{|c|c|c|c|c|c|c|}
\hline & & $N=5$ & $N=10$ & $N=20$ & $N=40$ & $N=80$ \\
\hline \multirow{2}{*}{ Limear } & $\begin{array}{l}14 \\
\% \\
\%\end{array}$ & $\begin{array}{l}368.3323 \\
2.55(-2)\end{array}$ & $\begin{array}{l}368.3823 \\
1.20(-22)\end{array}$ & $\begin{array}{l}368.4074 \\
0.52(-2)\end{array}$ & $\begin{array}{l}368.4200 \\
0.177(-2)\end{array}$ & \\
\hline & $\begin{array}{l}u \\
\% r \text { ree. }\end{array}$ & $\begin{array}{l}2.6635 \\
1.88(-2)\end{array}$ & $\begin{array}{l}2.6638 \\
0.7751(-2)\end{array}$ & $\begin{array}{l}2.6639 \\
0.375(-2)\end{array}$ & 2.6640 & 2.6640 \\
\hline \multirow{2}{*}{$\begin{array}{l}\text { Quaddrattic } \\
\text { Interpollation }\end{array}$} & 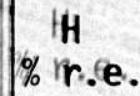 & $\begin{array}{l}368.33433 \\
2.50(-2)\end{array}$ & $\begin{array}{l}368.3832 \\
1.177(-2)\end{array}$ & $\begin{array}{l}368.4078 \\
(0.511(-22)\end{array}$ & $\begin{array}{l}368.4202 \\
0.117(-2)\end{array}$ & 368.4264 \\
\hline & ${ }_{\%} u$ & $\begin{array}{l}2.6636 \\
1.50(-2)\end{array}$ & $\begin{array}{l}2.6638 \\
0.751(-22)\end{array}$ & $\begin{array}{l}2.6639 \\
0.375(-2)\end{array}$ & 2.6640 & 2.6640 \\
\hline
\end{tabular}

TABLEE 6. 1 b Exilt Pressure Head alt Time of Wallwe Closure

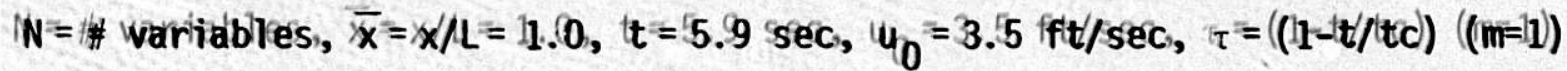

\begin{tabular}{|c|c|c|c|c|c|c|}
\hline & & $N=5$ & $N=110$ & $N=20$ & $N=40$ & $N=80$ \\
\hline $\begin{array}{l}\text { Linear } \\
\text { Inttempolration }\end{array}$ & $\begin{array}{c}1 H \\
\% \text { ir.ee. }\end{array}$ & $\begin{array}{l}391.91191 \\
36.0(-3)\end{array}$ & $\begin{array}{l}391.79773 \\
4.9(-3)\end{array}$ & $\begin{array}{l}391.77871 \\
0.23(-3)\end{array}$ & $\begin{array}{l}3911.77816 \\
0.092(-3)\end{array}$ & $\begin{array}{c}391.77789 \\
0.0\end{array}$ \\
\hline $\begin{array}{l}\text { Quadrraltic } \\
\text { Intterpolation }\end{array}$ & $\begin{array}{ll}14 \\
\%\end{array}$ & $\begin{array}{l}391.77937 \\
4.01(-3)\end{array}$ & $\begin{array}{l}391.78999 \\
3.04(-3)\end{array}$ & $\begin{array}{l}391.77834 \\
0.114(-3)\end{array}$ & $\begin{array}{l}391.77798 \\
0.046(-3)\end{array}$ & $\begin{array}{c}391.77780 \\
0.0\end{array}$ \\
\hline
\end{tabular}

TALLBE 6.IIC Pressure Head and Wellocity att $(\overline{(\bar{x}, t)}$

$N=\#$ wariablies, $\bar{x}=x / \mathrm{L}=0.2, t=77.7728 \mathrm{sec}, \psi_{0}=3.5 \mathrm{fft} / \mathrm{sec}, \tau=((1-\mathrm{t} / \mathrm{t} / \mathrm{tc}) \quad((\mathrm{m}=1))$

\begin{tabular}{|c|c|c|c|c|c|c|}
\hline & & & & & \\
\hline & & $N=5$ & $N=110$ & $N=20$ & $N=40$ & $N=80$ \\
\hline \multirow{2}{*}{$\begin{array}{l}\text { Limear } \\
\text { IInterpolalation }\end{array}$} & $\mid \begin{array}{c}14 \\
6 \% \text { r.ee. }\end{array}$ & $\begin{array}{l}288.31149 \\
211.7(-1-2)\end{array}$ & $\begin{array}{l}287.86544 \\
6.11(-22)\end{array}$ & $\begin{array}{l}287.83355 \\
5.0(-22)\end{array}$ & $\begin{array}{l}287.777700 \\
3.1(-22)\end{array}$ & \\
\hline & $\begin{array}{c}u \\
\% \text { in.e.e. }\end{array}$ & -0.2884862 & $\begin{array}{c}-0.2890388 \\
0.48\end{array}$ & $\begin{array}{c}-0.289272 \\
0.40\end{array}$ & $=0.28971199$ & -0.22901776 \\
\hline \multirow{2}{*}{$\begin{array}{l}\text { Quadtraltic } \\
\text { Intterpolitaltion }\end{array}$} & $\underset{614}{14}$ & $\begin{array}{l}288.11615 \\
118.4(-2))\end{array}$ & $\begin{array}{l}287.77825 \\
3.22(-2)\end{array}$ & $\begin{array}{l}287.77586 \\
2.4(-2)\end{array}$ & $\begin{array}{l}287.72225 \\
1 . .22(-2))\end{array}$ & 287.66894 \\
\hline & $\operatorname{lu}_{\%}^{4}$ & $\begin{array}{c}-0.2887322 \\
11.11\end{array}$ & $\begin{array}{c}-0.2898110 \\
0.221\end{array}$ & $\begin{array}{c}-0.2899928 \\
0.117\end{array}$ & $\begin{array}{c}-0.22901800 \\
0.085\end{array}$ & -0.2290428 \\
\hline
\end{tabular}


For convergence we would expect these differences to be decreasing. This is mot thappening yet in the liinear case, lbut may be starting in the quadratitic case. In any case, iff we do thave convergence iit lis clear that we are not in the asymptotic range where a rate of convergence can be detternined.

In liziter sections we will be comparing warious approximate solutions; Thence iit is desirable to establiish benchmark wallues. As the benchmark wallues, we initend to use the method of characteristivics with $/ N=80$ and quadratic initerpolvation. In an altempt to estimate the number of sirgnificicant diigits that we are justifified in using for these lbenchmark wallues, we willi consiider the effect of limear extrapolation on the pressure and wellocitity. For cexamplee, consiider Tablie $6.11 \mathrm{c}$, and extrapollate hivnearly these wallues, then we cobtain the follinowing table.

TABLE 6.IId Litnear Extrapoliation of Pressure Head and Wellocilty

\begin{tabular}{|c|c|c|c|c|}
\hline N & TH & $\begin{array}{c}H \\
\text { (fextrapollated })\end{array}$ & u & $\begin{array}{c}\text { u } \\
(\text { (extrapolratted })\end{array}$ \\
\hline 5 & 288.11615 & & $=0.287322$ & \\
\hline 110 & 287.77825 & 287.04035 & -0.289810 & $=0.292298$ \\
\hline 20 & $237 . .77586$ & 287.777735 & $=0.289928$ & -0.290046 \\
\hline 40 & 287.77225 & 287.66864 & $-? / 20180$ & $=0.290432$ \\
\hline
\end{tabular}

The extrapollatted lpressure 287.66864 can be compared with the benchmark pressure of 287.6894 ((see Table 6.11 ) from which we infeer that we can thave some conffiddence in four siignifficant diigitts for the benchmark pressure. When we compare the cextrapoliated wellocitity - 0.2290432 with the lbenchmark wallue -0.290428 , we inffer that we can thave conffidence in three ssignifficant dirgitts for the tbenchmark welocitty.

In Trable 6.22 we ipresent the carse of $\mathrm{im}=22$. Here, as we can ssee, the

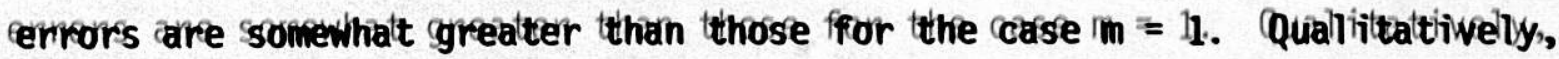
the comparisson tbetween livinear and quadtrattic initempolation, as welly as the

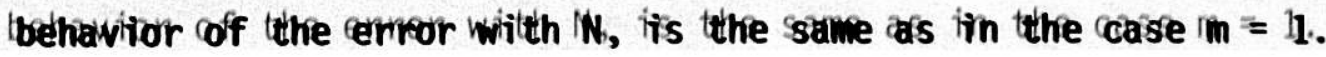


TAABLE 6.2a Pressure Head and Velocitty alt $(\bar{x}, t)$

$N=\#$ wariables, $\bar{x}=x / L=0.6, t=2.1477 \mathrm{sec}, u_{0}=3.5 \mathrm{ft} / \mathrm{sec}, \tau=\left((1-\mathrm{t} / \mathrm{t} / \mathrm{tc})^{2}((\mathrm{~m}=2)\right.$

\begin{tabular}{|c|c|c|c|c|c|c|}
\hline & & $N=5$ & $N=110$ & $N=20$ & $N=40$ & $N=80$ \\
\hline \multirow{2}{*}{$\begin{array}{l}\text { Linear } \\
\text { IInterpollattion }\end{array}$} & $\begin{array}{l}\text { H } \\
\% \text { rr.e. }\end{array}$ & $\begin{array}{l}427.11372 \\
4.07(-22)\end{array}$ & $\begin{array}{l}427.2366 \\
1.774(-2)\end{array}$ & $\begin{array}{l}427.2858 \\
0.590(-2)\end{array}$ & $\begin{array}{l}427.3103 \\
0.295(-2)\end{array}$ & 427.3225 \\
\hline & $\frac{u}{4}$ & $\begin{array}{l}1.8880 \\
3.118(-2)\end{array}$ & $\begin{array}{l}1 . .8883 \\
1 . .59(-2)\end{array}$ & $\begin{array}{l}1.8885 \\
0.530(-2)\end{array}$ & 11.8886 & 1.88886 \\
\hline \multirow{2}{*}{$\begin{array}{l}\text { Quadraltic } \\
\text { Interpoliation }\end{array}$} & $\begin{array}{l}\text { H } \\
\% \text { ir.e.e. }\end{array}$ & $\begin{array}{l}427.11433 \\
3.93(-2)\end{array}$ & $\begin{array}{l}427.2393 \\
1.68(-22)\end{array}$ & $\begin{array}{l}427.2872 \\
0.557(-2)\end{array}$ & $\begin{array}{l}427.31110 \\
0.279(-2)\end{array}$ & 427.3229 \\
\hline & $\begin{array}{c}\text { u } \\
\% \text { ir.e. }\end{array}$ & $\begin{array}{l}11.8881 \\
2.65(-2)\end{array}$ & $\begin{array}{l}11.88844 \\
1.06(-2)\end{array}$ & $\begin{array}{l}1.88885 \\
0.530(-2)\end{array}$ & $\begin{array}{c}1 . .8886 \\
\ldots\end{array}$ & 11.88886 \\
\hline
\end{tabular}

TABLE 6.2b Exilt Pressure Head alt Time of Walive Closure

$N=\#$ wariables, $\bar{x}=x / L=11.0, t=5.9 \mathrm{sec}, u_{0}=3.5 \mathrm{fft} / \mathrm{sec}, \tau_{\tau}=((1-t) / \mathrm{tc})^{2}((\mathrm{~m}=2))$

\begin{tabular}{|c|c|c|c|c|c|c|}
\hline & \\
\hline & & $N=5$ & $N=110$ & $N=20$ & $N=40$ & $N=80$ \\
\hline $\begin{array}{l}\text { Linnear } \\
\text { Interpollatition }\end{array}$ & $\begin{array}{c}H \\
\% \text { ir.e. }\end{array}$ & $\begin{array}{l}301.3029 \\
18.6(-22)\end{array}$ & $\begin{array}{l}300.77 / 82 \\
1.116(-22)\end{array}$ & $\begin{array}{l}300.77585 \\
0.509(-2)\end{array}$ & $\begin{array}{l}300.77489 \\
0.2466(-2)\end{array}$ & 300.77441 \\
\hline $\begin{array}{l}\text { Quadrattic } \\
\text { Interpolatition }\end{array}$ & $\begin{array}{c}\mathrm{H} \\
\% \text { ir.ee. }\end{array}$ & $\begin{array}{l}300.77768 \\
11.112(-22)\end{array}$ & $\begin{array}{l}300.77538 \\
(0.353(-2))\end{array}$ & $\begin{array}{l}300.74466 \\
0.1113(-2))\end{array}$ & $\begin{array}{c}300.77432 \\
0.057\end{array}$ & 300.77415 \\
\hline
\end{tabular}

TABBLE 6 2C Pressure Head and Welrocity att $(\overline{(\bar{x}, t)})$

$N=$ wariabless, $\overline{\bar{x}}=x / \mathrm{L}=0.2, t=77.7728 \mathrm{ssec}, 4_{0}=3.5 \mathrm{ft} / \mathrm{sec}, \tau_{t}=\left((1-1 \mathrm{t} / / \mathrm{tcc})^{2}((\mathrm{~m}=2))\right.$

\begin{tabular}{|c|c|c|c|c|c|c|}
\hline & \\
\hline & & $i N=5$ & $N=110$ & $N=20$ & $w=40$ & $N=80$ \\
\hline \multirow{2}{*}{$\begin{array}{l}\text { Limear } \\
\text { Internpollattion }\end{array}$} & $\mid \begin{array}{ll}14 \\
\% \\
\text { ir.ee. }\end{array}$ & $\begin{array}{c}308.10662 \\
0.7720\end{array}$ & $\begin{array}{c}306.39222 \\
0.1173\end{array}$ & $\begin{array}{c}306.27763 \\
0.1135\end{array}$ & $\begin{array}{c}306.0665 \\
0.1106\end{array}$ & 305.8535 \\
\hline & $\operatorname{lu}_{\%} \mid$ & -0.294277 & -0.3122257 & -0.3138211 & -0.315837 & $-0.3117 / 713$ \\
\hline \multirow{2}{*}{$\begin{array}{l}\text { Quardrattioic } \\
\text { Intrempollattion }\end{array}$} & $\mid \begin{array}{c}1 H \\
\% \text { ir.ee. }\end{array}$ & $\begin{array}{c}307.46994 \\
0.525\end{array}$ & $\begin{array}{c}306.08826 \\
0.071144\end{array}$ & $\begin{array}{c}305.9967 \\
0.04333\end{array}$ & $\begin{array}{c}305.8642 \\
0.040\end{array}$ & 305.774119 \\
\hline & $\mid \begin{array}{l}u \\
x \rightarrow r . e e .\end{array}$ & $\begin{array}{c}-0.304867 \\
4.01\end{array}$ & $\begin{array}{c}-0.3155688 \\
0.7700\end{array}$ & $\begin{array}{c}-0.31163774 \\
0.383\end{array}$ & $\begin{array}{c}-0.3177590 \\
0.3334\end{array}$ & -0.3118653 \\
\hline
\end{tabular}




\section{COMPARISON OF GALERKIN, LLAX, AAND MMETHOD OF CHARAACTERISTIICS}

comparison of Severall Gallenkin Approximations

We begin thiis section lby comparing warious Gallerkin approximations to the water hammer probliem. Recall) from section 5 thalt $k$ cdenotes the corder ((degree $=\mid k-1)$ ) of the pirecewise pollynomialls lused in the Gallenkin approximattion.

Furthermore, for any $k$, we can choose any wallue of $w, 0 \leq w \leq l k-1$ where $w$ it the smoothness of the pirecewise pollynowialis. Thalt iis, iff $f(x)$ iis a pirecewise pollynomiall with smoothness index w, then if $(v-1))((x))$ is continuouis. IIf the hength $L$ of the piipe iis divivided inter \& subiintervalis, then we see that we thave the three parameters $((k, v, l)$ at cour diisposall when generating Galienkin approximattions to the water hammer probliem. For a given sett $((k, w, k))$, the number of wariables ((for eitther the velocilty lu or the ipressure thead $H$ ) willit be

$$
N=k+((k-v))((l-11))
$$

With the three parametters alt cour disisposall we can generalte a warietty of approximattions to the watter hammer problem. We willil sttart the mumericall sttudy by comparing the folltowing ssive Galkerkion approximationis.

$k=4, v=3 \ldots$. Pirecewise cubic pollynowialls with continurous ssecond derivathives. These are the swooth cubicic splimes.

$k=4, v=2 \ldots$. Pirecewise cubic pollynowizalls with conitimuous ffirsst deriwaltives. These are the Hemitite cubicic splimes.

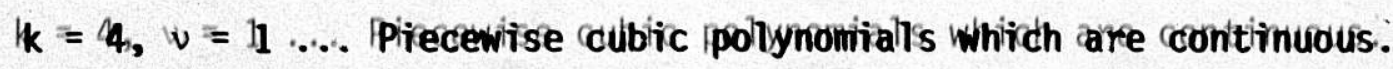

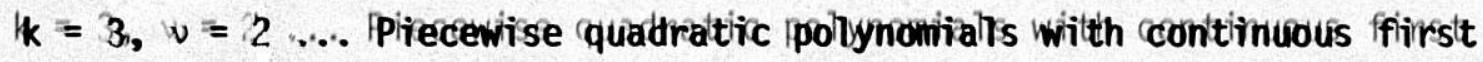
cderivaltives ((smooth quardratitics)).

$k=3, w=11 \ldots$... Pirecewise quadtrattic pollymonizalls which are contimuous.

$k=2, w=11 \ldots$. Pirecewise liinear pol nynowizalls whiich are continuous.

Simce we are usiing an CODE soliver [[1]1] tro solive the syystem of cordinany

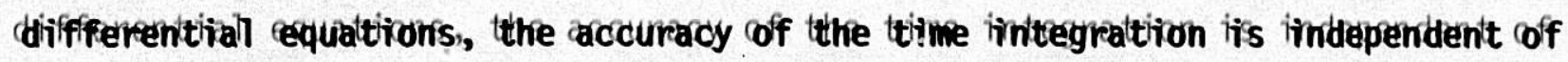
the accuracy of the spativall alpproximation. Hence we wish to ffirx the raccuracy of

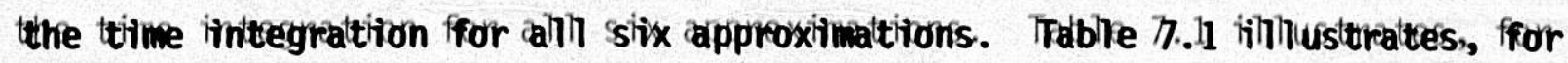
cour partivicular approximattion, the effifect of wanying the trolerance in the lrocall

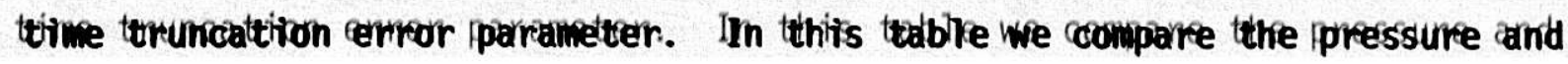


TFABLE 7..1 Effect of Time Integraltion Tollerance in ODE Ifor 'Gailerkin Approximat tion with $k=3$, $v=1, l=2, t=7.728$

\begin{tabular}{|r|c||c|c|c|}
\cline { 2 - 5 } \multicolumn{1}{c|}{} & IEPS $=110^{-16}$ & IEPS $=116^{-9}$ & LEPS $=110^{-16}$ & IEPS $=110^{-19}$ \\
\hline $\bar{x}=.2$ & 289.4342 & 289.4387 & -0.270184 & -0.270207 \\
. .4 & 2772.0870 & 272.68871 & -0.2677719 & -0.267764 \\
.6 & 286.0881 & 256.0824 & -50.258748 & -0.258733 \\
.8 & 248.5941 & 248.5933 & -0.1159387 & -0.159363 \\
\hline
\end{tabular}

wellocitity at ffour Mocaltions in the ipipe wiith a toolrerance, IEPS $=110^{-16}$, and IEPS $=110^{-9}$ in the $O D D E$ sollwer. IFrom thiis ttable we innfer thalt the cerror (due to

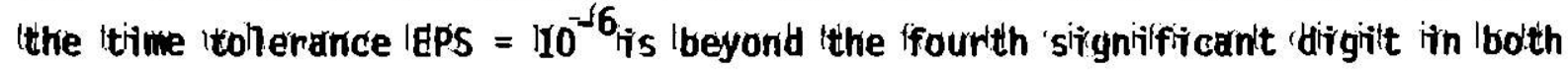
the ipressure and the welocilty. In comparing the 'siix 'Galkerkion approximaltions we thave Iused IEPS $=110^{-6}$ iunder the assumption thalt the cerror idue to tiime irnte'graltion is lbeyond the fourth sirgnifficicant dirgilt for lbo'th the Ipressure and the

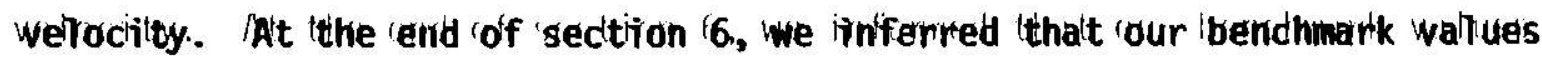

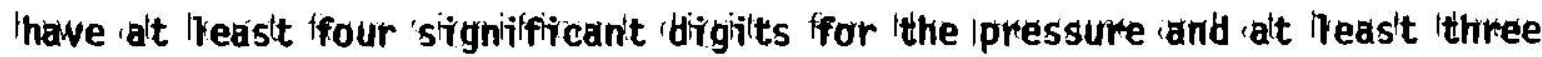
sizgniffircant diigilts ffor the wellociitty. Thus we feel justriffied in lusiong EPS $=110^{-16}$ in the (Gallerkion approximaltions.

IFor the comparioson of the sive Galrerkion approximaltions, we iproceed as

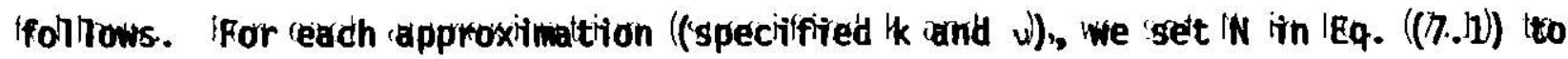

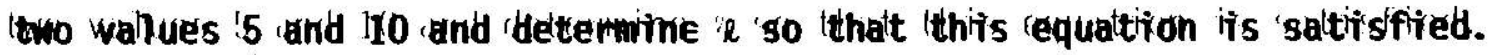

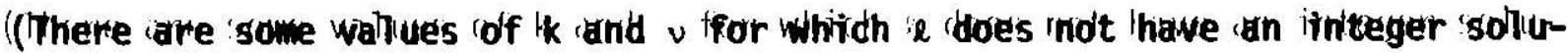

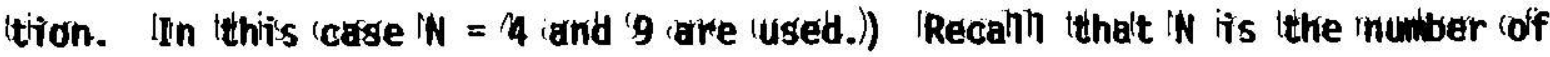

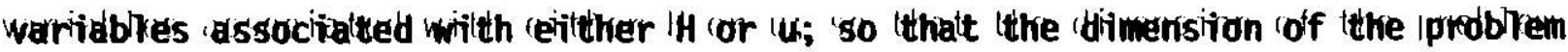

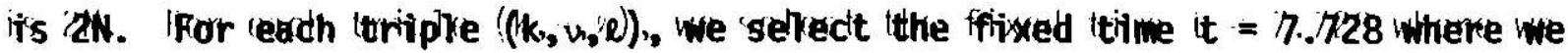

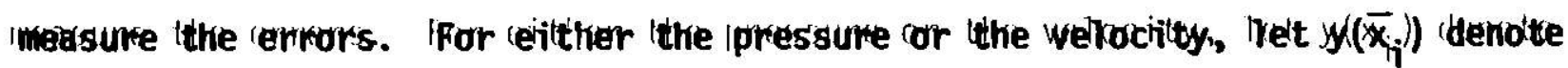

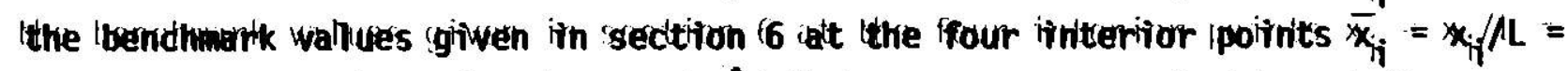
$(0.2,0.4,0.6,50.8$ callong the ipipe. If ssame pointts, we compute the ffolhowing (quanitivitives. 


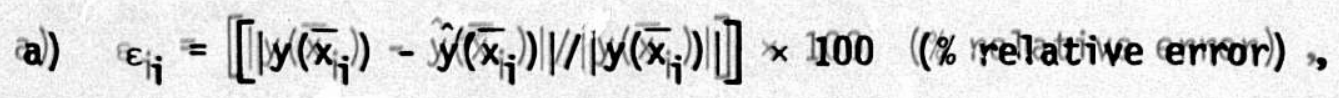

$$
\begin{aligned}
& \text { b) } \left.\varepsilon_{\max }=\max \left\{\varepsilon_{i j}: 11 \leq i \leq 4\right\}\right\} \text {, } \\
& \text { (c) } \varepsilon_{\text {mean }}=\frac{11}{4} \sum_{i=1}^{4} \varepsilon_{i j} \text {, } \\
& \text { (d) } \varepsilon_{\text {ir.jm.s. }}=\left[\frac{11}{4} \sum_{i=1}^{4} \varepsilon_{\varepsilon_{i i}}^{2}\right]^{\frac{1}{2}} \text {. }
\end{aligned}
$$

For the ipressure and the wellocitty, these quantititives are given iin the frollirowing two tables.

TABLLE 7..2a Enro is in the Pressure at $t=77.7228$

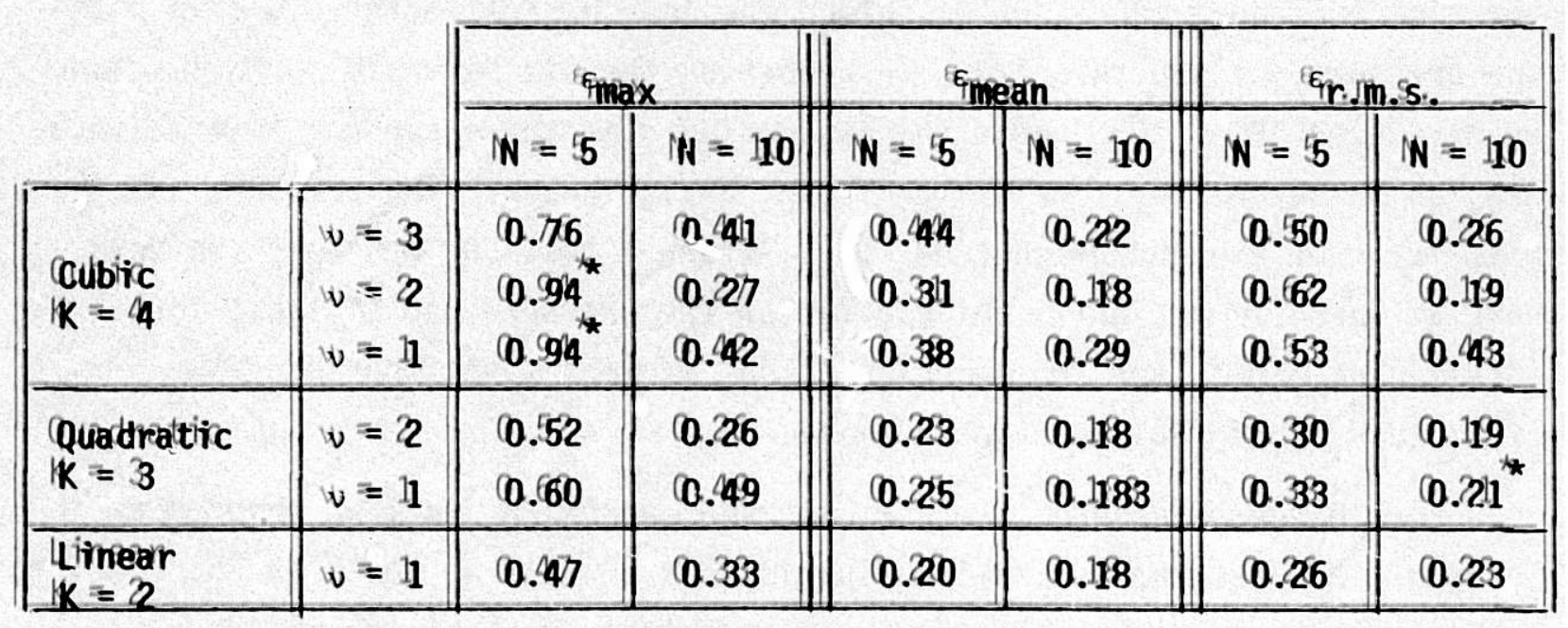

TABLE 7..20 Enroors in the Wellocity att $t=77.7728$

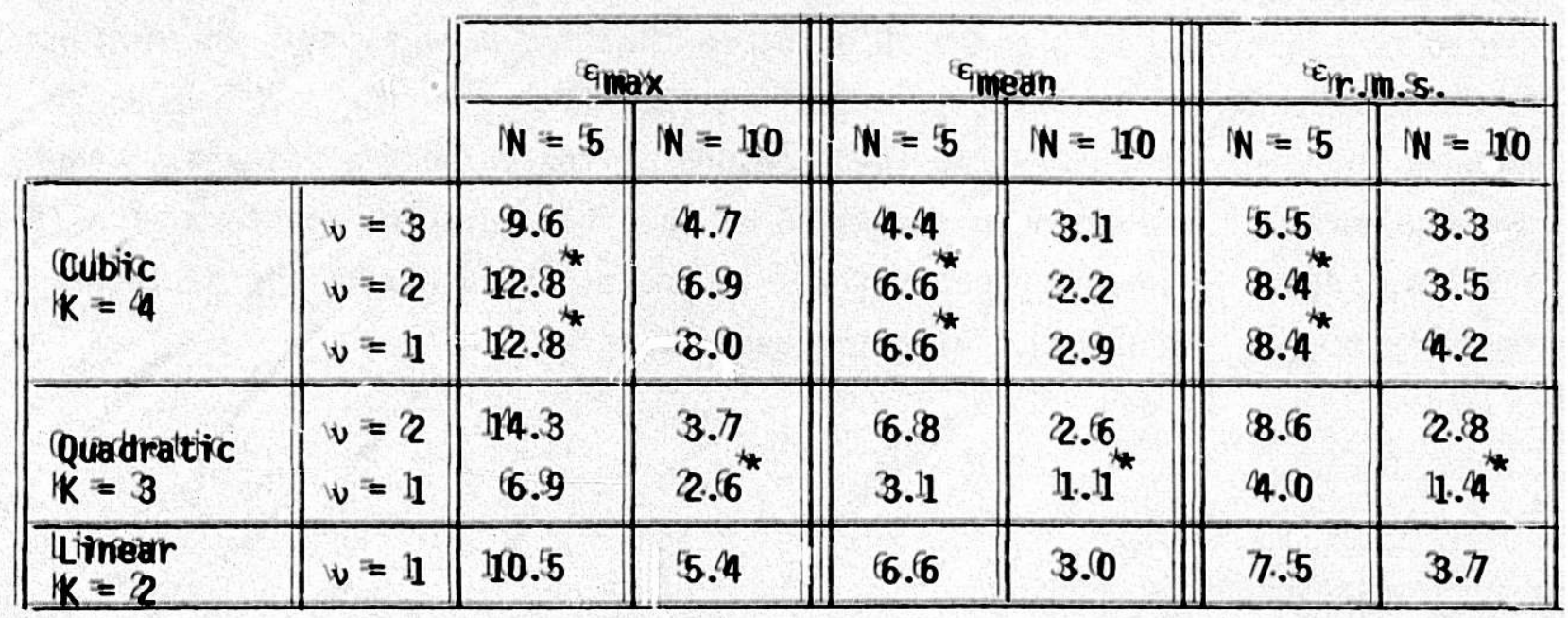

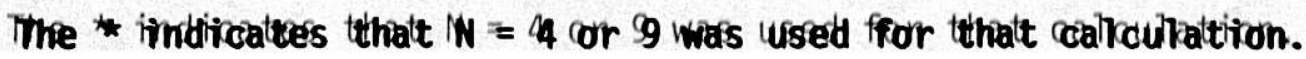


Based con the datta in thiis table, we draw the folliowing conclusions.

- As far as the pressure iis concerned, no cone particular approximation sittands cout from the resit.

- As far as the wellocitty its concerned, the continuous quaddratio $((k=3, \nu=11)$ sittands cout from the resit. Moreover, the diffiference its siigniifficant; the error its generalliny liess than lhallf thalt for any of the cother approximations.

- The uise of thizigh corder approximaltions such as cubbics appears to offifer mo advantage for iprobliems of thiis toype

From the rresullts in Tablie 77.22, we ssellect two Gallenkitn alpproximaltions for comparison witth the Method of Charactterisstizcs and the Laax Method. The con-

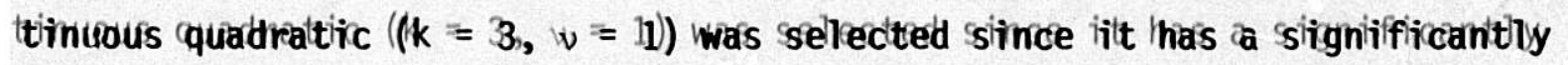
smalliker exroor for a given number of wariablies than any otther Galkerkin approximaltion. The continuous liinear $((k=2, w=11)$ was selkected tbecause iit thas the ssame assymptottic cerror in the spaltival approximation as the method of

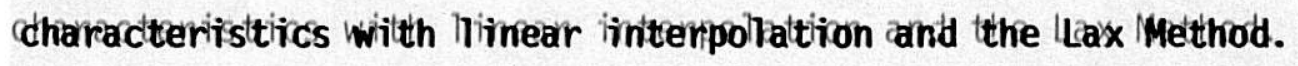

We mext consizder the ratte of convergence of these two Galinerkin alpproximattions as the sspattiall mesh its reefined. Using $\varepsilon_{\text {max }}$ and $\varepsilon_{\text {mean }}$ as idefined ion

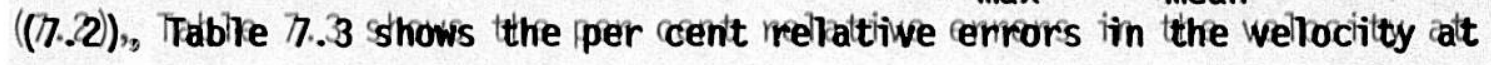
$t=77.7728$.

TABLLE 77.3 Eerrors in the Welrocitty att $t=77.7728$

\begin{tabular}{|c|c|c|c|c|c|c|c|c|}
\hline \multirow[b]{3}{*}{, } & \multirow{2}{*}{\multicolumn{4}{|c|}{$\varepsilon_{\max }$}} & & & & \\
\hline & & & & & \multicolumn{4}{|c|}{ Emean } \\
\hline & $N=5$ & $N=110$ & $N=220$ & $N=37$ & $N=5$ & $N=110$ & $N=20$ & $N=37$ \\
\hline Quadratticic $((k=3, w=11))$ & 16.9 & $2.66^{*}$ & $11.06^{*}$ & 0.57 & 3.11 & $11 . .11^{*}$ & $0 . .53^{*}$ & 0.27 \\
\hline Limear $((k=2, v=11))$ & 110.5 & 5.4 & 11.86 & & 6.6 & 3.0 & 0.999 & \\
\hline
\end{tabular}


From Table $7 . .3$ we draw the follthowing conclussions.

- Both approximaltions appear to tbe converging.

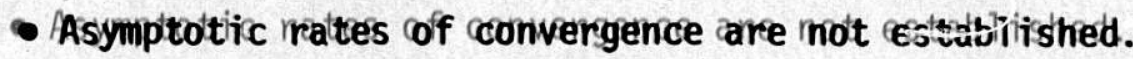
Esitimaliting raltes, we fiond:

\begin{tabular}{l|l|l||l}
$k=3, w=11$ & 11.5 & 0.92 & 11.11 \\
\hline$k=2, w=11$ & 11.11 & 11.6 &
\end{tabular}

Aat best iit would appear that the assymptotic ratte may tbe about cone ffor eitthar of these approximattions.

- Enror for the quadratitic $((K=3, w=11)$ iis abouit thallf the errrar for the livinear approximaltion.

\section{Compariison witth Method of Charactteriisitics and Lax Methrod}

We mow compare the methot of charactterisitiocs, Gallenkin, and the LLax

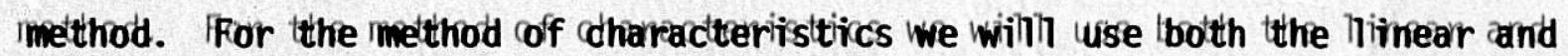

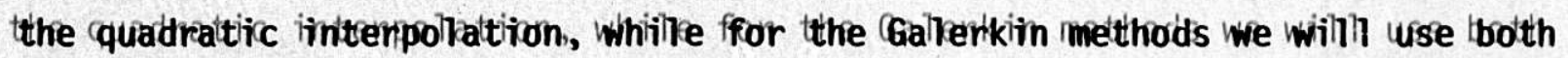
the quardratitic $((k=3, w=11)$ ) and the liinear $((k=2, w=11))$. Thus cour compariisson willth involive ffive approximatitions. The compariison willth the con the basits of the enror in the wellocitity alt $t=77.7728$ and alt the four initerivor poinits

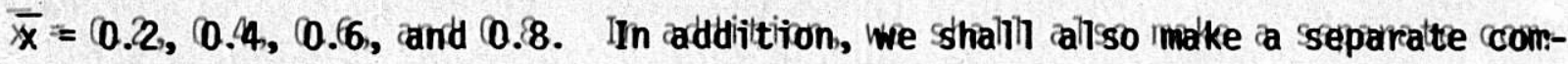
ppariison at the resenvoiir end of the piipe $\bar{x}=0.0$ which irs, cof course, a tboundany ppoinit.

In Trable 77.44 we compare the enrors alt the interior poinits for $1 N=55,110$,

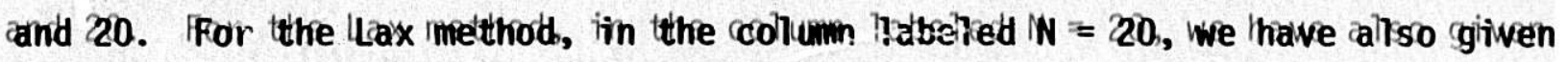
the enror for $N=40$. 
TARBLE $7 . .4$ Errors in the Welocitty at $t=7.7728$ at Some Interior Points, $m=11$

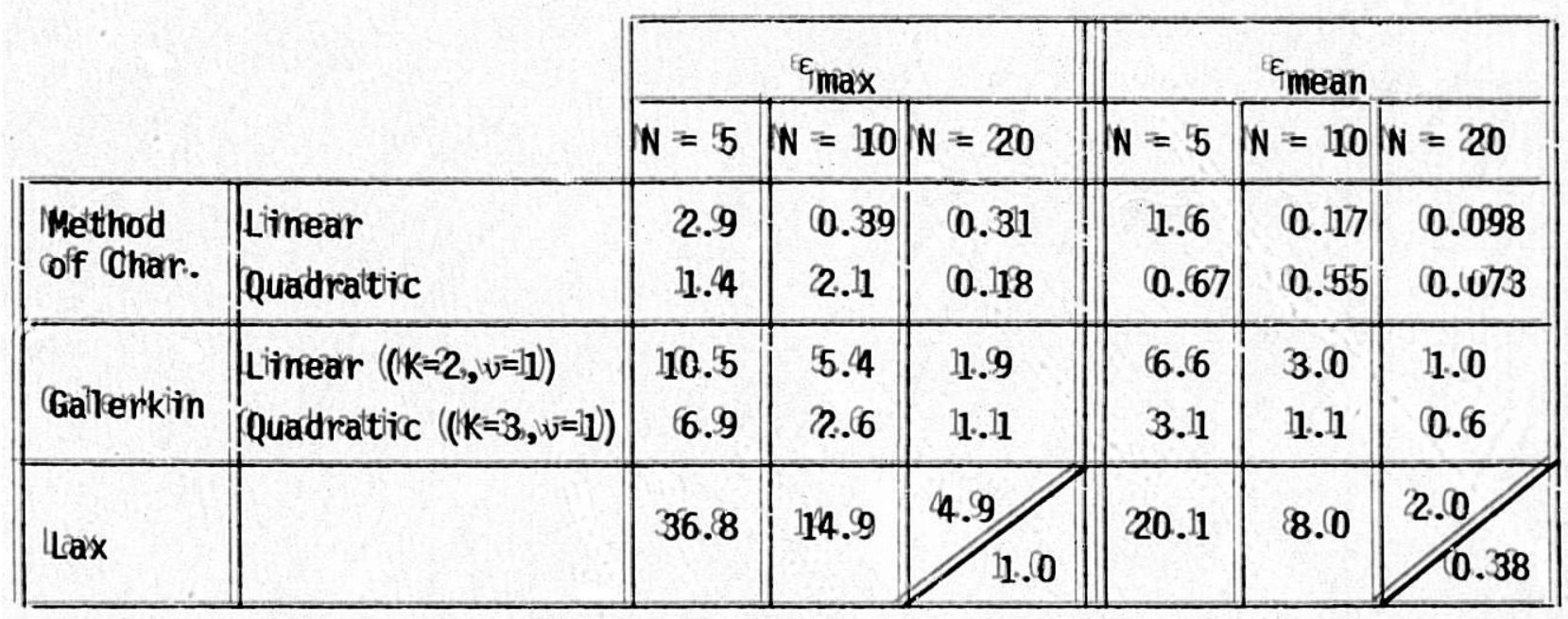

TABLEE $7 . .5$ Errors ion Welrocity att Reservoiir att $t=7.7728, \mathrm{~m}=11$

\begin{tabular}{|c|c|c|c|c|c|c|c|}
\hline & \\
\hline & & $N=5$ & \begin{tabular}{|l|}
$\%$ \\
r.8e. \\
\end{tabular} & $N=110$ & $\begin{array}{l}\% \% \\
\% .8 .\end{array}$ & $N=20$ & r.e. \\
\hline $\begin{array}{l}\text { Metthod } \\
\text { of Charr. }\end{array}$ & $\begin{array}{l}\text { Limear } \\
\text { Quadratitic }\end{array}$ & $\begin{array}{l}-0.290528 \\
-0.297282\end{array}$ & $\begin{array}{l}0.37 \\
1.995\end{array} \mid$ & $\begin{array}{l}-0.2911675 \\
-0.29119940\end{array}$ & 0.03 & $\mid \begin{array}{l}-0.22911629 \\
-0.2911654\end{array}$ & $\begin{array}{l}0.004 \\
0.02\end{array}$ \\
\hline Galkenkion & $\begin{array}{l}\text { Limear }((K=2, w=11) \\
\text { Quaddrattic }(K=3, w=11)\end{array}$ & $\begin{array}{l}-0.2222270 \\
-0.3324467\end{array}$ & $\begin{array}{l}23.8 \\
111.3\end{array}$ & $\begin{array}{l}-0.288953 \\
-0.2903114\end{array} \mid$ & $\left|\begin{array}{l}0.911 \\
0.444\end{array}\right|$ & $\begin{array}{l}-0.2295564 \\
-0.2289659\end{array}$ & $\mid \begin{array}{l}1.366 \\
0.666\end{array}$ \\
\hline Lax & & -0.4939976 & 50.9 & -0.258461 & $111 . .4$ & $\mid-0.300397$ & 3.0 \\
\hline \multicolumn{4}{|c|}{$\begin{array}{l}\text { Benchmartk wallure }=0.2911594 \\
\text { ((MinoD, Quad, } N=80) \text { ) }\end{array}$} & \multicolumn{2}{|c|}{$\angle$ Lax, $I N=40$} & -0.22912228 & 0.113 \\
\hline
\end{tabular}

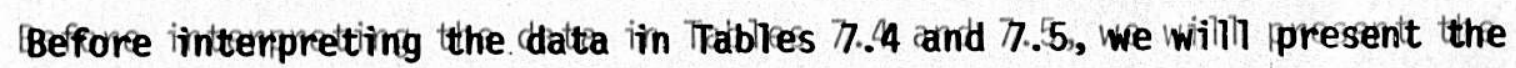
same tyype of datta for the carse $1 m=2$. We may motte thatt in thiis carse the toransizent responses are more ssevere than iin the ccase $m=11$. IIn iparticiculizar, there are fow flow reversalls in the course of the theansitent. The ffirsit reverssall coccurs alt approximaltelly $t=3.864 \mathrm{ssec}$. and the ssecond alt the ustual time

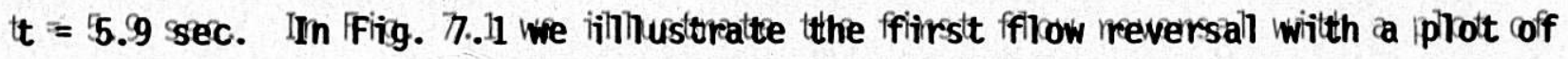
welrocititives as a ffunction of posiition alt ttime $t=3.864 \mathrm{sec}$. ffor the ccases im $=11$

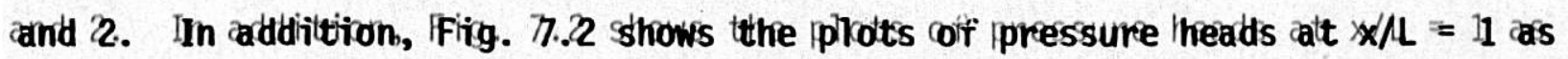

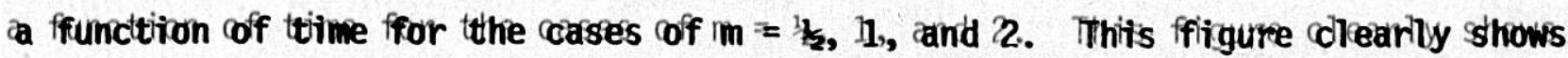

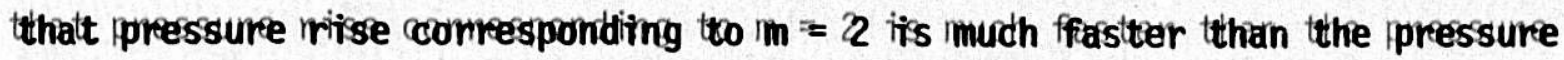
riise correspontiong to $\mathrm{im}=1$. 


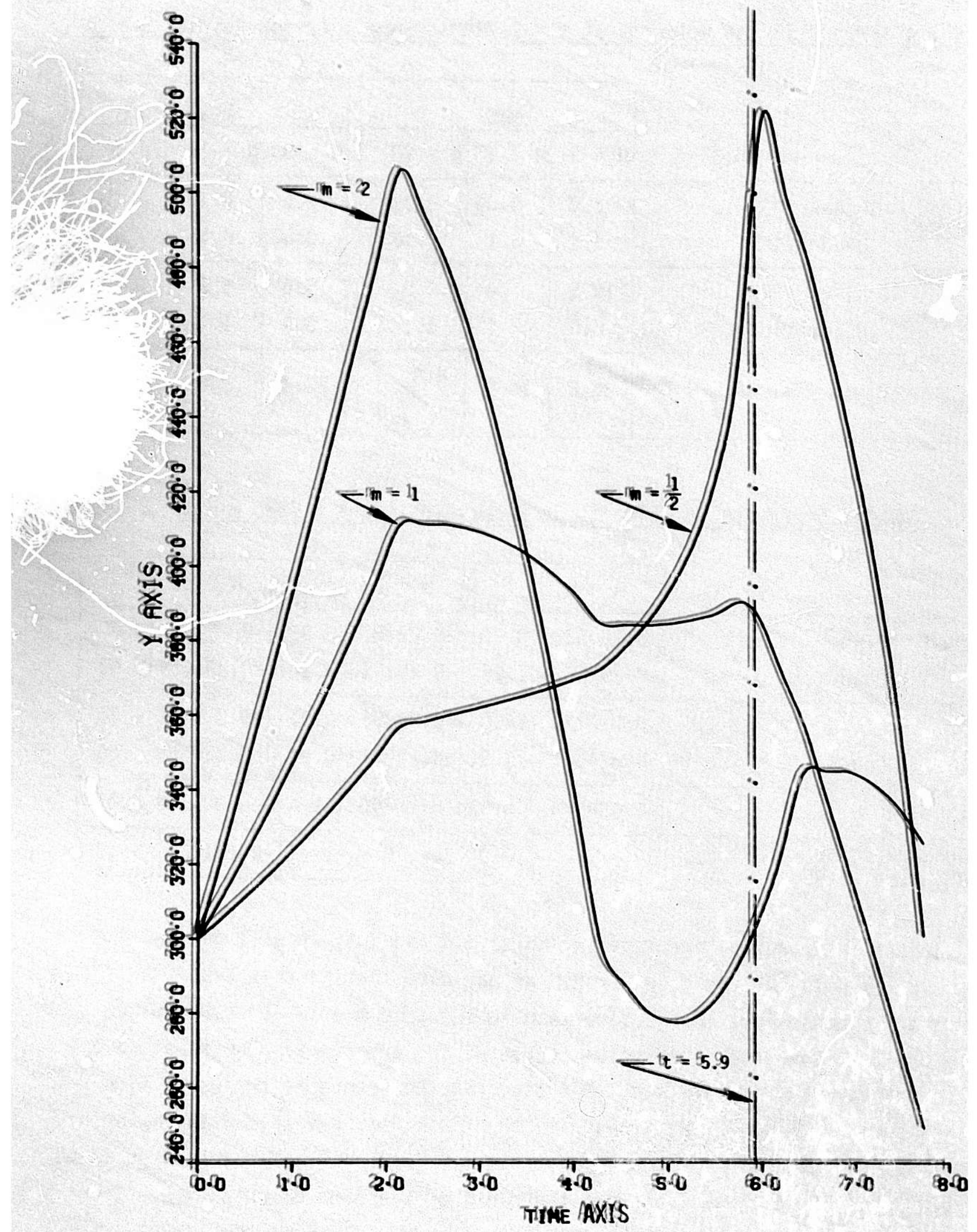

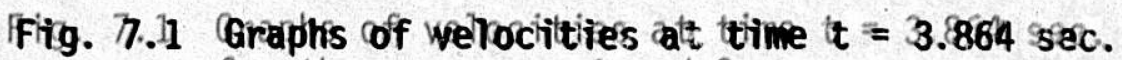
for the carses $m=11$ and 2 


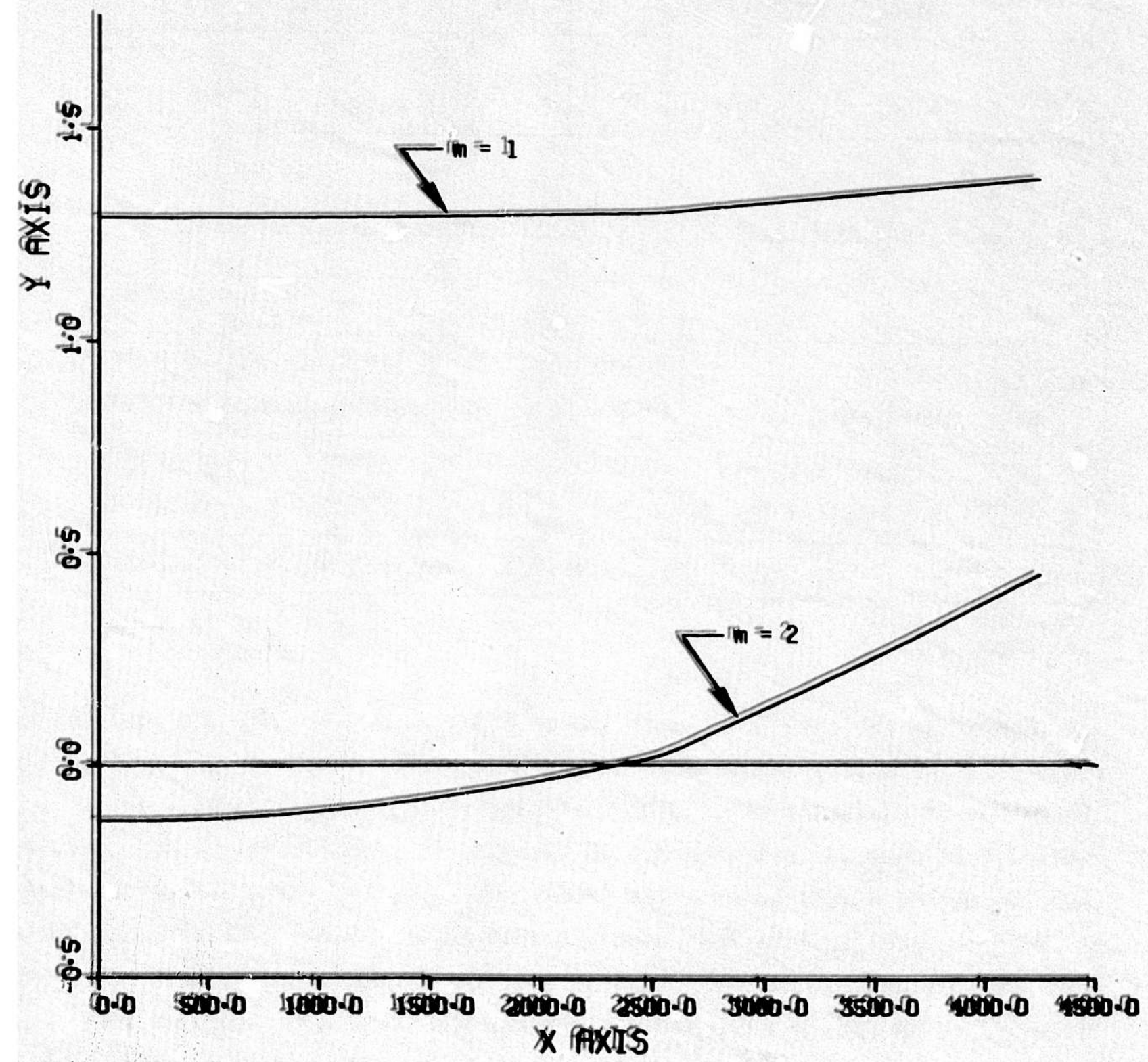

Fiig. Th.2 Graphs of exilt pressures as faunctions of toime for the carses $i n=3,2,1$, and 2 
TABLE 7.6 Emrors in Velocitty att $t=77.728$ alt Some Interior Proinits, im $=2$

\begin{tabular}{|c|c|c|c|c|c|c|c|}
\hline & \multirow{2}{*}{\multicolumn{3}{|c|}{$\xi_{\max }$}} & \multirow{2}{*}{\multicolumn{3}{|c|}{ Emean }} \\
\hline & & & & & & & \\
\hline & & $N=5$ & $N=110$ & $N=20$ & $N=5$ & $N=110$ & $N=20$ \\
\hline \multirow{2}{*}{$\begin{array}{l}\text { Wethrod } \\
\text { of Char. }\end{array}$} & Linnear & 7.7 & 2.0 & $1 . .5$ & 2.3 & 0.57 & 0.41 \\
\hline & Quadrattic & 4.3 & 11.0 & 0.72 & 11.4 & 0.36 & 0.23 \\
\hline \multirow{2}{*}{ Galkerkin } & Linear $((K=2, v=11))$ & 116.0 & 6.0 & 1.99 & 6.0 & 3.7 & 0.67 \\
\hline & Quadtratio $((K=3, v=11)$ & 77.6 & 2.9 & 11.4 & 3.2 & 2.3 & 0.72 \\
\hline $\operatorname{Ltax} x$ & $a_{0}$ & 36.3 & 117.77 & $\begin{array}{l}8.0 \\
3.7\end{array}$ & 27.3 & $111 . .2$ & $\begin{array}{c}3.0 \\
1.11\end{array}$ \\
\hline
\end{tabular}

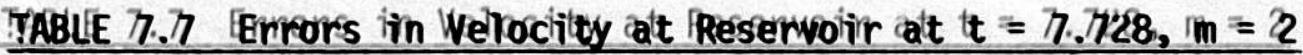

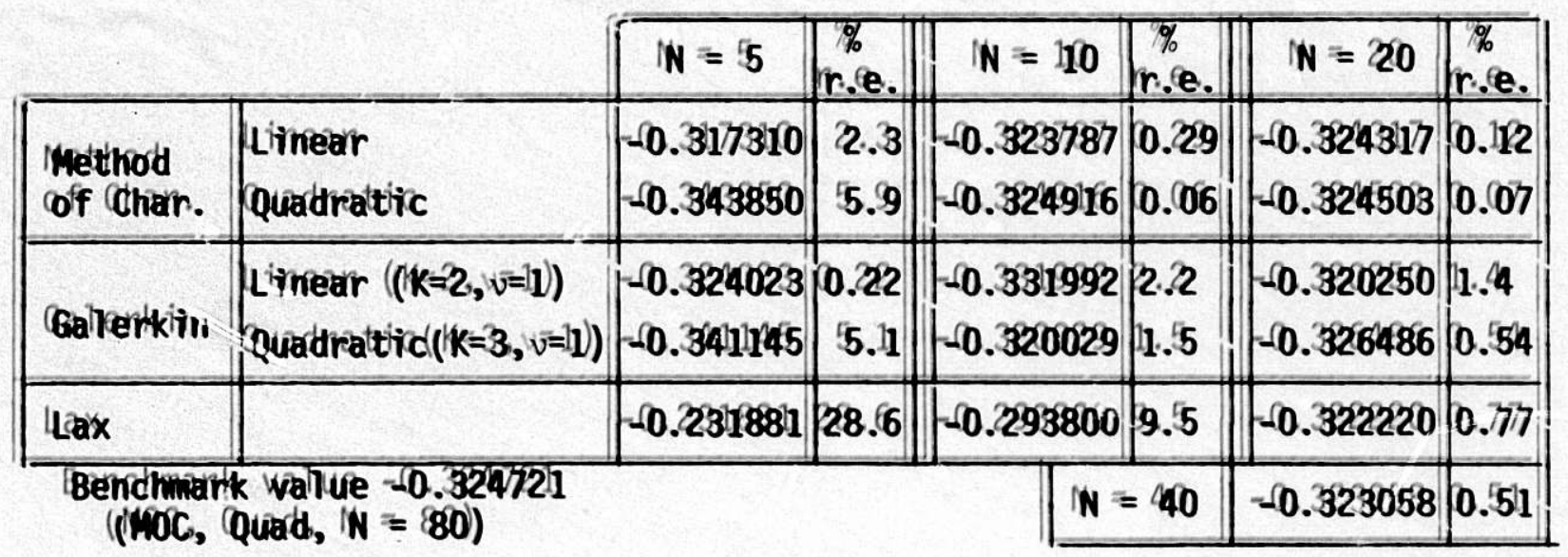

comparing the ccase $/ m=11$ with the case $/ m=22$, we see thalt the quallititaltive

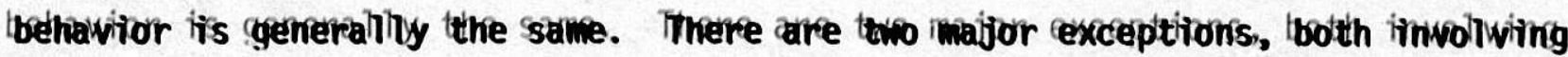

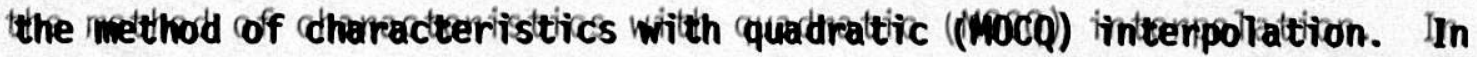

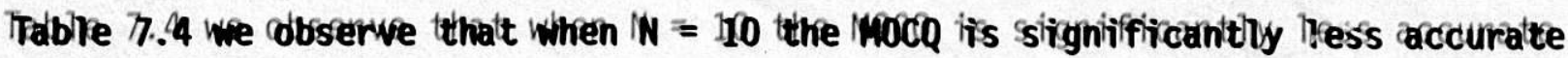

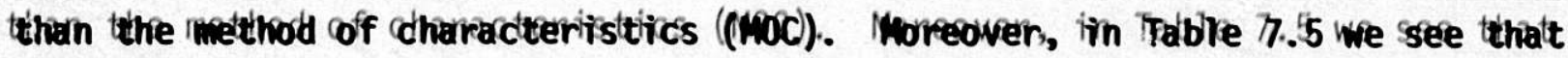

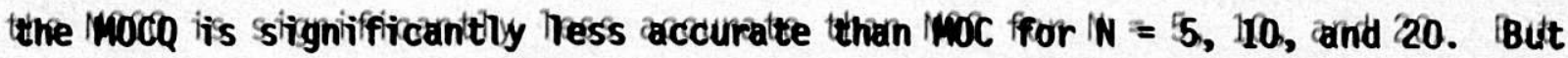

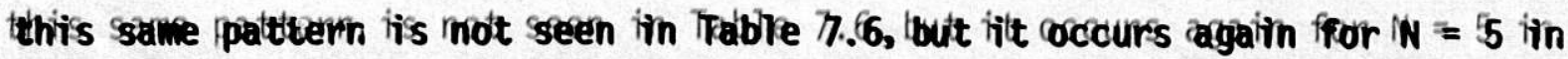

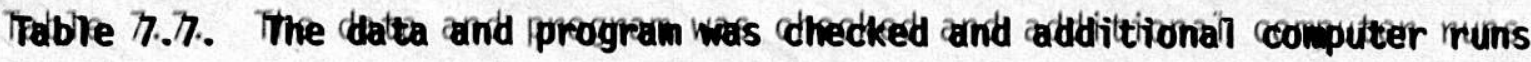

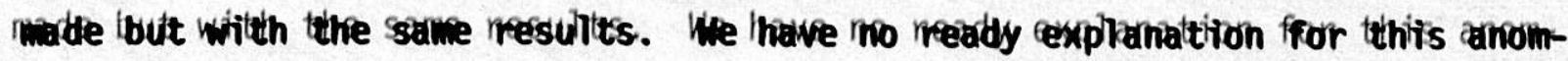
allous tbethavizor. 
We interpret the data in these tables as folliows.

- For a given wallue of $\mathbb{N}$, the method of characteristics (liinear or quadratio) is significicantly imore accurate than (Galerkin procedures -- generallily twice as accurate.

- For a given wallue of $\mathbf{N}$, Gallerkin procedures are significicantlly more accurate than the Lax Imethod $=$ generallily more than towice as accurate.

- For a given wallue of IN, the Lax method was surprisingly iinaccuratte. For example when $\mid N=5$, iits maximum relative error at initerior points was alt lreast $36.8 \%$ which is more than 26 times the relattive error for IMocQ. Assmptoticaliny, the Laax Imethod, Gallerkin in linear, and IMOC alli have spatiall approximations of the same corder; buit for moderate vallues of $1 \mathrm{~N}$, the actuall errors dififfer ssigniificanthy.

- The Gallerkin methods were solved with an CODE soliver; thus they represent an example of the luse of hiigh corder implicicit tiime integration in a hyperbolic systtem. From these tables, we ffeel that Gallerkin teechniques offer mo substantivall advantage cover the Lax Imethod. We base this juldgement on the folltrowing consiiderations.

a) As IN increases, the "rated" of enror reduction is mot veny dififferent for Gallerkin and Lax.

b) We have not shown the titimes for these calloulations for tho reasons.

((ii) Preciise ttimes are not readiliny availlkable; thus we coulid inot accurattelyy compare MOC, MOCQ, and Lax. They ali trake about the same time.

((iii)) The Galkenkin method was dorne witth a generall punpose ppackarge; whereas the cothers were done with specivall punpose coodes. Thus we dird not know how tro arccuratelyy access the coverhead in the parkacge. However, in an efffortt tro substantitiate cour conchusition

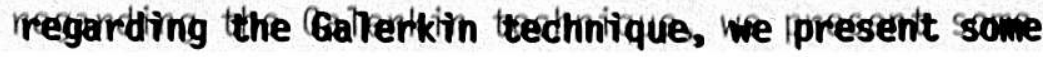


representative COPU times for these callculations. The times are in seconds on an IIBM $370 / 1395$, and represent the entive job cost.

TABLE 7.8 CPUU Times for Warious Methods

\begin{tabular}{|c|c|c|c|c|c|}
\hline & $N=5$ & $N=110$ & $N=20$ & $N=40$ & $N=88$ \\
\hline MOC & 1 & 11 & 2 & 4 & 114 \\
\hline MocQ & 1 & 1 & 2 & 7 & 26 \\
\hline Gall. Litn. & 8 & 113 & 45 & & \\
\hline Gall. Quard & 9 & 118 & 51 & & \\
\hline Lax & 11 & 1 & 2 & 5 & \\
\hline
\end{tabular}

Even with a substanitiall allhowance for coverhead, the Gallerkim procedures do mot appear competittive with even the Laax method for hypperbolitic problems of this type.

- The rmethod of characteriisticics, either livinear cor quadraticic, i is lby far the best method for Iproblems off this toype. This concliussion takes intito arcountit the ssimpliicitty of the method, accuracy, and computatitionall cosit. 


\section{THE CCASE IOF/A RITGID WALL}

Ceritain 'speciffic imethods 'such a's Lax-Wendrofff 'scheme $[[7,8]$ and the dorior

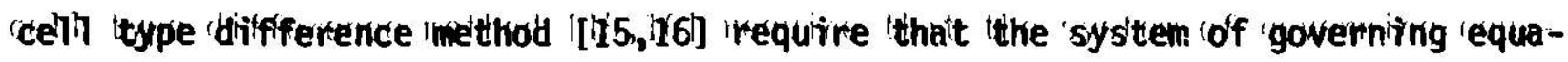
titions be ion a conservaltive form. 'Since the case of an celasitic waill does inot read to a conservaltive form coff governing cequaltions, we are cobliged to sltubly the

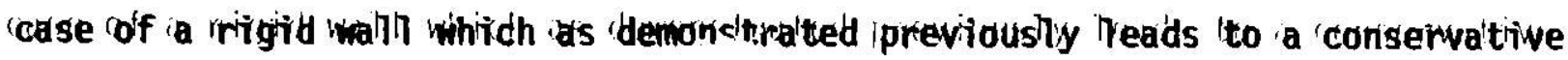
Iform of the system of lEqs. ((2.114): und ((2.115) ((with boundany conditions 'given by

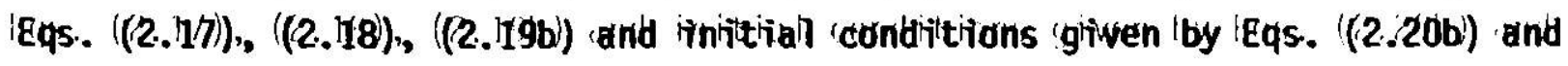
$((2.80 \mathrm{c})))$.

IIt iis worth molting that the systten $((2.7))$ and $((2.110)$ is inot lequivalken't to the system ((2.174) and ((2.155)). To loriing cout the 'differencess we iproceed ats ifolltrows. Ifrom lEq. ((2.113c)), we thave

$$
\left.i_{p}=q_{R} g\left(1++C_{R}\right)\right) / R^{i 2} ;
$$

thus

$$
\frac{\partial p}{\partial t}=\frac{q_{R}(g)}{a^{2}} \frac{\partial H}{\partial t} \text {, sand } \frac{\partial p}{\partial x}=\frac{q_{R} g}{a^{2}} \frac{\partial H}{\partial x} \text {. }
$$

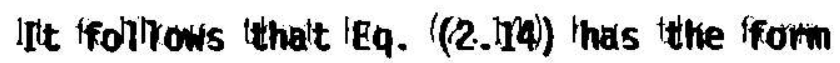

$$
\frac{\partial H}{\partial t}+u \frac{\partial H}{\partial x}+\frac{Q^{2}}{g} \frac{p}{g_{R}} \frac{\partial u}{\partial x}=0 \text {, }
$$

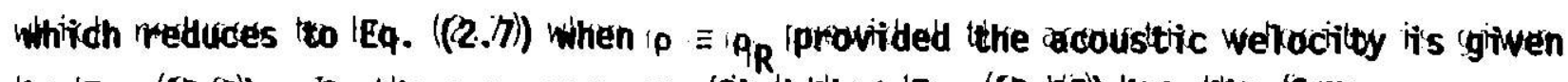

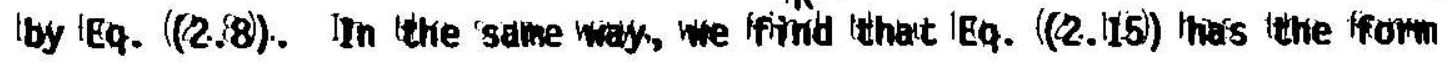

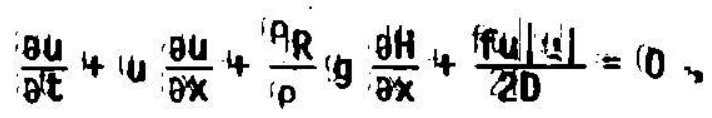

Whiidh is leq. ((2.1)10) when ip $\equiv q_{R}$.

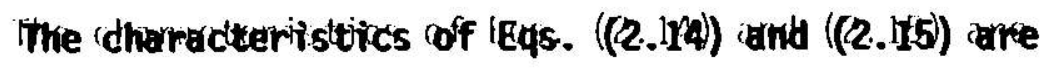

$$
\lambda=(6 / \rho \pm \pm \quad(f=\omega \pm \pm(a)),
$$

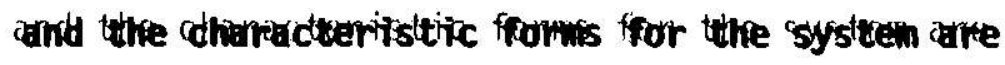



a) $\frac{d x}{d t}=c / 1 p+a$

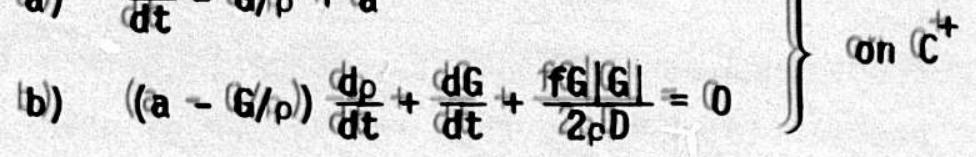
a) $\frac{d d x}{d t}=\left(\sigma / h_{p}-a\right.$
b) $\left.\left.=((a+c) / p)) \frac{d p}{d t}+\frac{d d G}{d t}+\frac{f f G|G|}{2 p D}=0\right)\right\} \cos C^{-}$

In corder to obttain the benchmark wallues we use the method of characteriistivics with limear interpolation. The firnitite difiference approximations of these equations its the same as described in ssection 3 fror the carse of liimear initerpolatition. 


\section{THE WWO-STEP ILAX-WENDROFF IDYFFERENCE SCHEME}

The two-sitep (Lax-Wentro'ff 'scheme ((cff. $[[7]], \mid[8]])$ is cone of the inost ipopu-

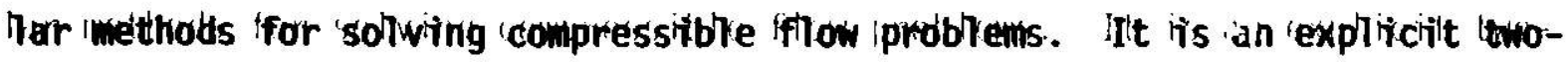
step method ibased con ia second corder Tayllor serijes cexpansion in time; thereby cgeneraltiong secont corder accuracy in tiine. When applized to sysitems colf hyperIbolitic cequaltions ion consenvaltive form, the scheme can lbe (described as ffollhows. Lett us corrsitter the system

$$
\frac{\partial w}{\partial t}+\frac{\partial}{\partial x} l B((x, t, t w)=\mid F(x, t, w)
$$

Where $w=w(x, t)$ is a wedtor wailued ffunction of the spattial wariable $x$ and teime $t$, cart is and if are wecteor wallued ffundtions off the indiicalted arguments.

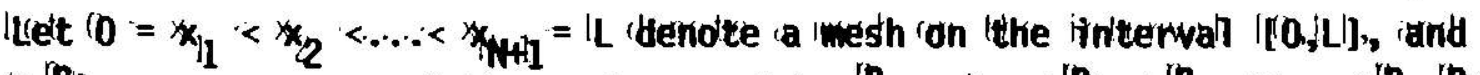

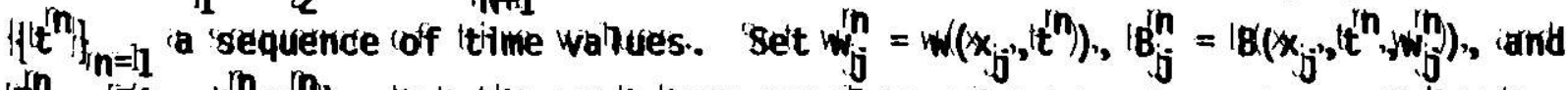

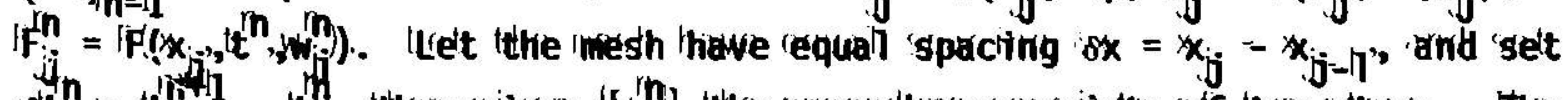

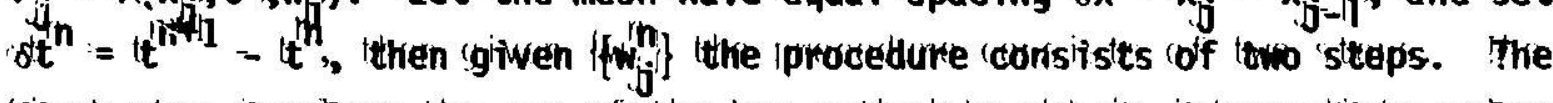

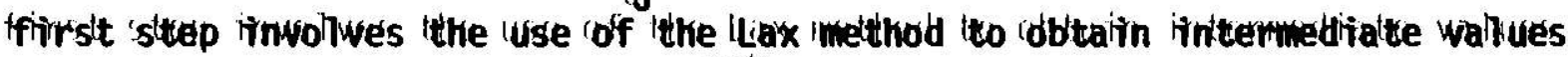

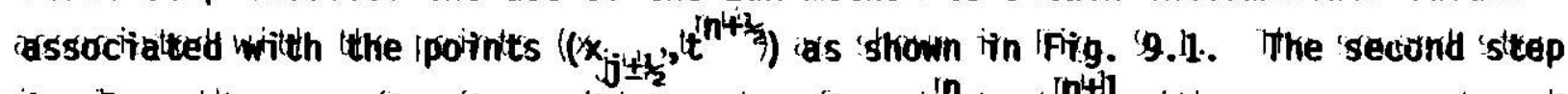

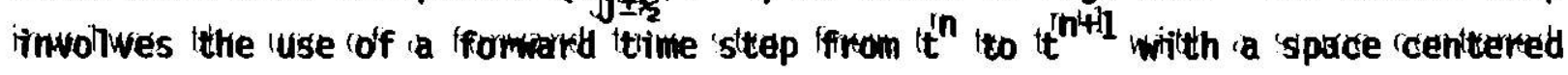
idiffference capproximaltion lusing the initermedialte wallues firom lthe ffirsit sitep.

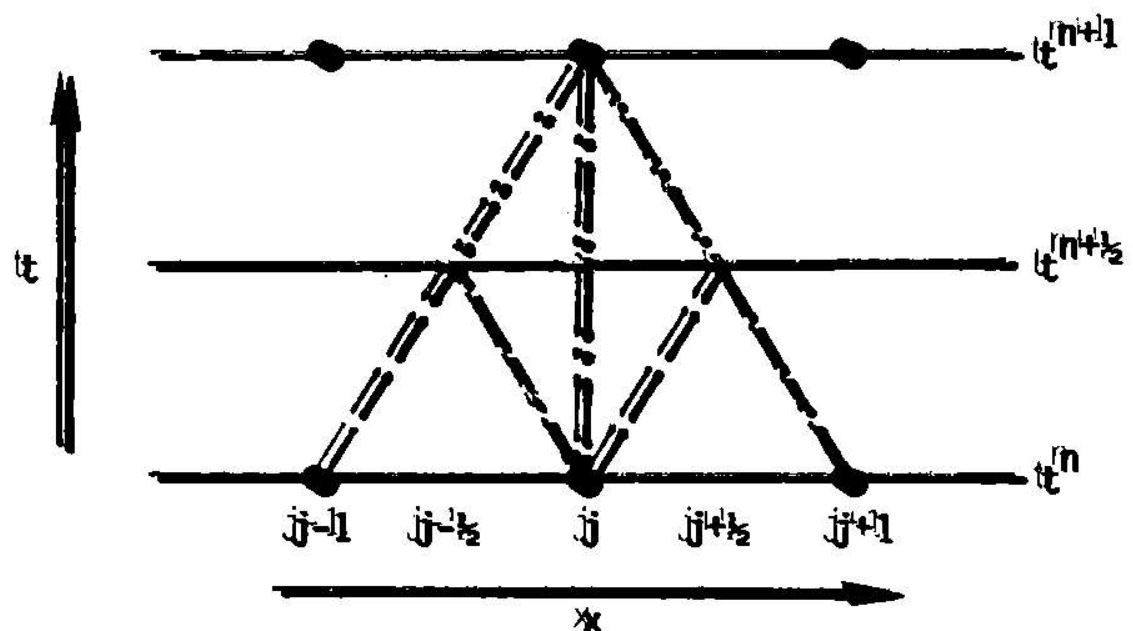

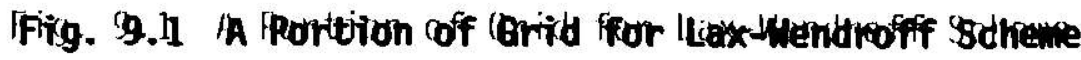


For a system of the form $\left((9.11)\right.$, the ffirsit sttep in fiindiing $w_{j j}^{n+1} w_{i i l l l}$ ibe to callculatte the intemmediatte wallues

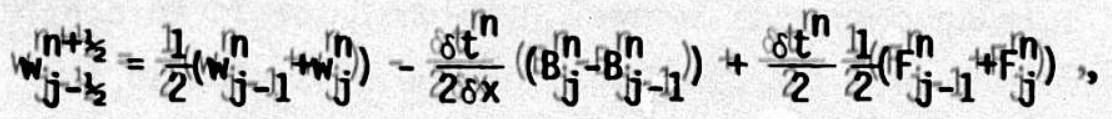

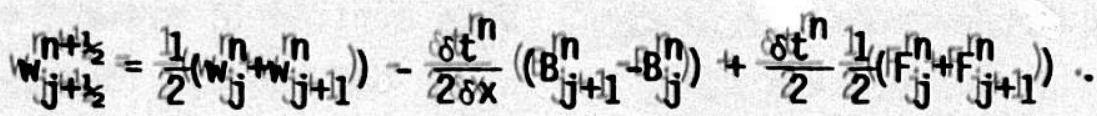

Then deffine

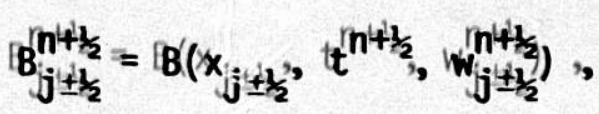

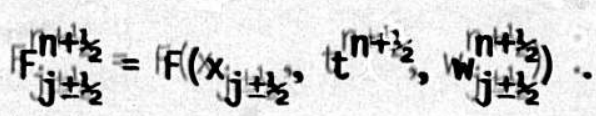

The ssecont sttep is then given tby

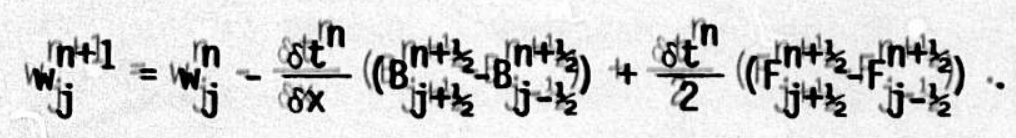

Diffiference Equationis

In cour applicication, $w=\left((p, 96)^{\prime \prime}\right.$, and from $((2.5))$ and $((2.173 c)$ we thave

$$
\frac{\partial p}{\partial x}=p_{R} g \frac{\partial H}{\partial x}=a^{2} \frac{\partial p}{\partial x} ;
$$

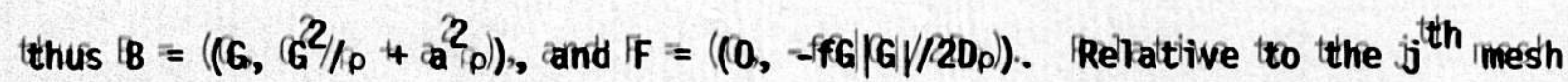
poinit, the firirstt strep takes the from

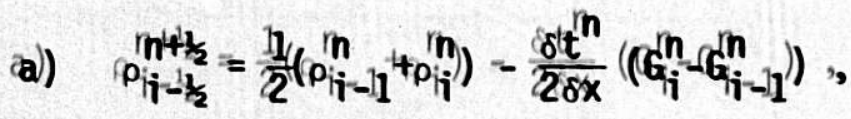

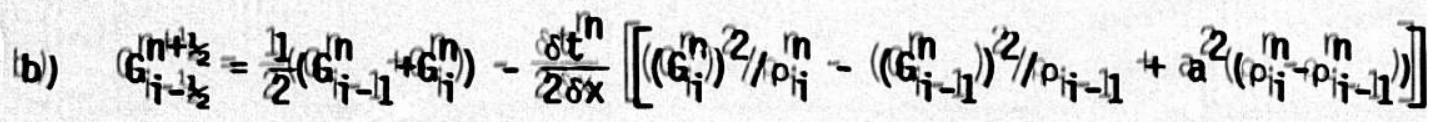

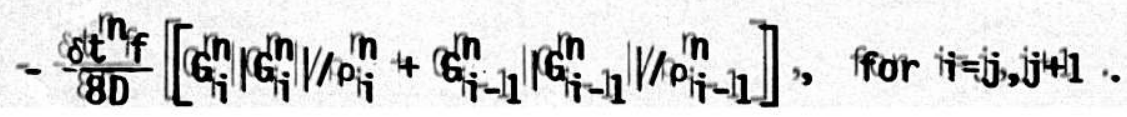


The second stepp takes the form

$$
\begin{aligned}
& \text { a) } \quad \rho_{j j}^{n+11}=\rho_{j j}^{n}-\frac{\delta t^{n}}{\delta x}\left(\left(G_{j j+\frac{1}{2}}^{n+1 / 2}-G_{j-1 / 2}^{n+1 / 2 / 2}\right)\right.
\end{aligned}
$$

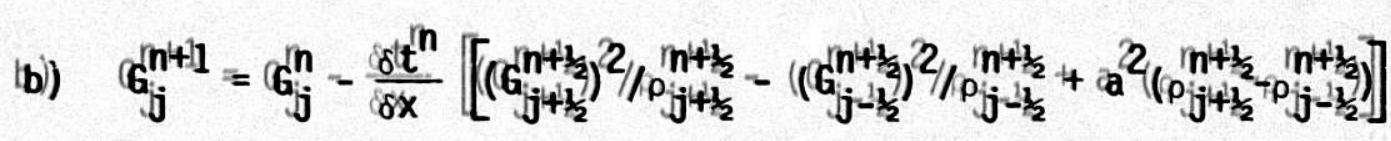

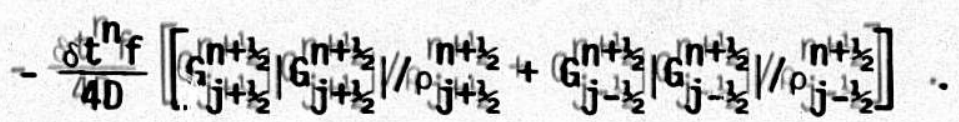

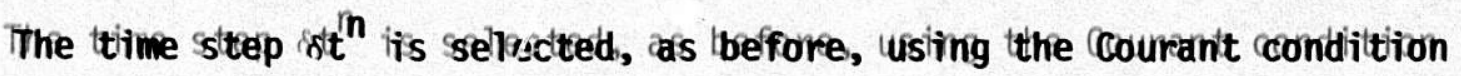

$$
\left.\delta t^{n} \leq \delta x / \lambda\left(a+|| u^{n} \mid\right)\right)
$$

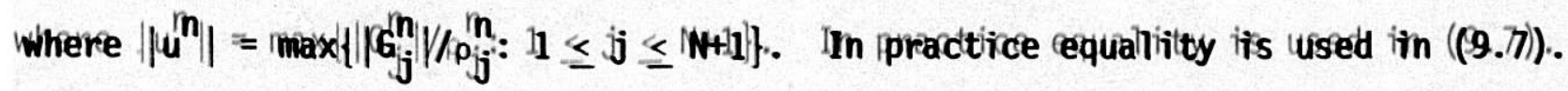

\section{Boundany condititions}

The two-sttep Lax-Wendroffif is appliicable alt the interior pointes lbut mot

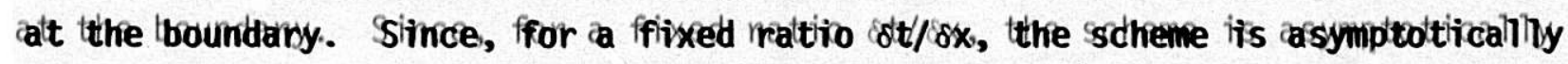
ssecond corder in sit, the terealtment' alt the boundarizes should attitemptt to mainitain

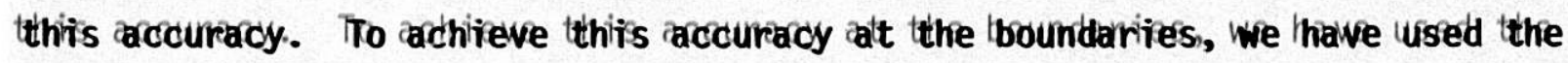
methrod off characteriisteics. The procedure can tbe descritibed as frolliows.

\section{Leefit Bounidany}

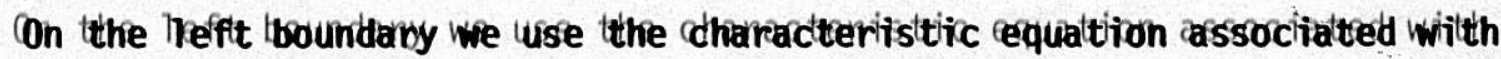
the $C^{-}$cunve deffined ion Eq. ((8.2)). Thus on $C^{-}$, we thave

$$
\begin{aligned}
& \text { a) } \frac{d d x}{d t}=(G / 10-a d, \\
& \text { (b) }-((a+c G / p)) \frac{d p}{d t t}+\frac{d d G}{d t}+\frac{f f G|G|}{2 p d D}=0 \text {. }
\end{aligned}
$$

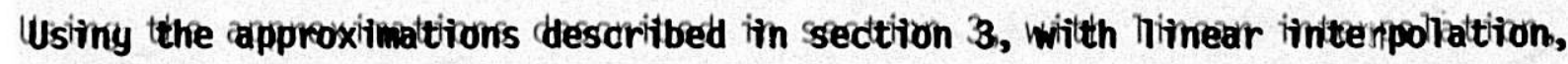
we ffintd

$$
\begin{aligned}
& \text { a) } P_{11}^{n+11}=P P O R_{P} \text {, }
\end{aligned}
$$

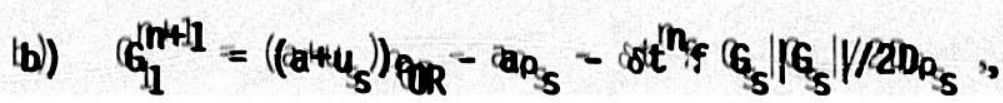


Where

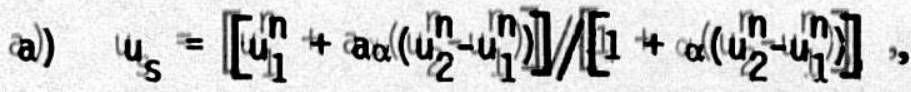
b) $P_{s}=P_{O R}+\alpha\left(\left(a-u_{s}\right)\right)\left(\left(P_{2}^{m}+p_{O R}\right)\right.$,
c) $G_{s}=\operatorname{las}_{s} 4 \psi_{s}$,
(d) $a=\operatorname{sot}^{n} / / 18 x$.

\section{Riightit Boundany}

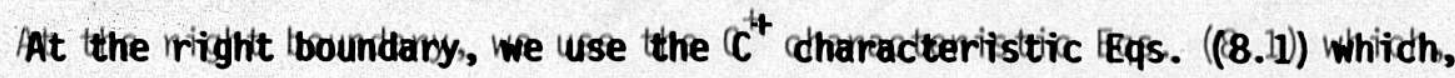

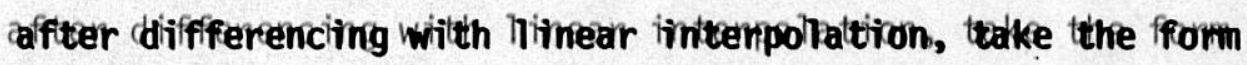

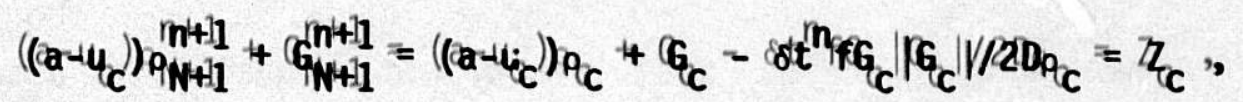

Where

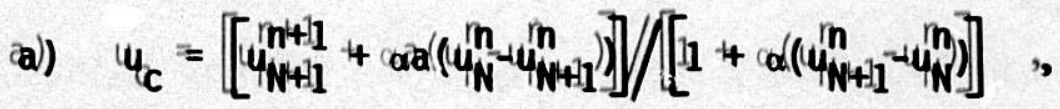

$$
\begin{aligned}
& \text { b) } \left.p_{c c}=q_{w+1}^{m}+\alpha_{d}\left(a_{a}+u_{c}\right)\right)\left(\left(q_{N}^{m}+q_{w+1}^{m}\right)\right) \text {, }
\end{aligned}
$$

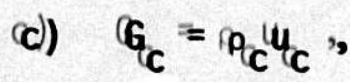

$$
\begin{aligned}
& \text { (d) } a=\operatorname{sit}^{n} / / 18 x \text {. }
\end{aligned}
$$

The tboundany conditition ((2.117)) iis writtiten itn the from

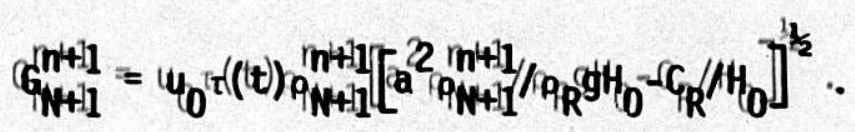

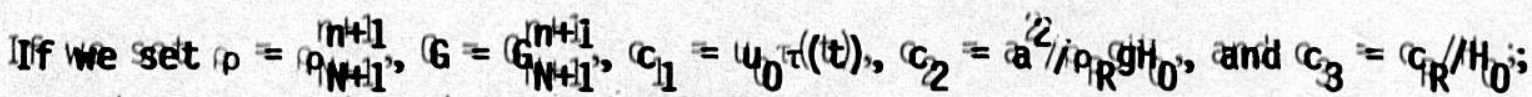
EEqs. ( $(9.111)$ ) and ( $(9.113)$ thave the from
a) $\left(\left(a-y_{c}\right)\right)_{p}+c=\frac{\pi}{c_{c}}$
b) $\quad\left(G=c_{1 p} p\left[\left[c_{25} p-c_{3 f}\right]^{\frac{1}{2}}\right.\right.$

or

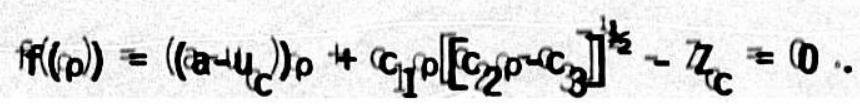


This equation iis solived usiing the INewton-Raphson iiteraltions

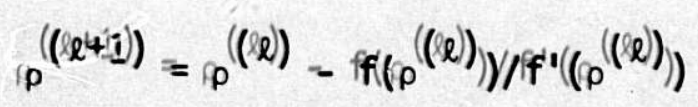

with
a) $\quad p^{((0))}=q_{\left(n+11^{\prime}\right.}^{((n)}$, and
b) $f^{\prime}((p))=a+u_{c}+\left[\left[c_{11}\left(\left(3 c_{2} 2^{\left.p-22 c_{3}\right)}\right) / p_{2}\left(\left(c_{2} 2^{p-c_{3}}\right)^{1 / 2}\right]\right.\right.\right.$.

The iitterationions are sttopped when

$$
\left|11-p((l e+11)) / / p p^{((l))}\right| \mid<110^{-s 9} ;
$$

whereupon

$$
p_{p h+1}^{r n+11}=p^{((l l+1))}
$$

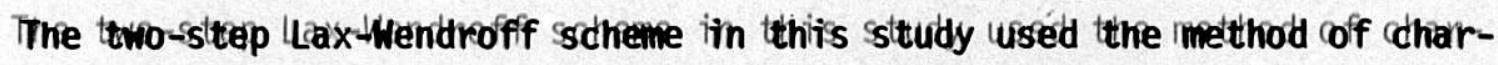
acteriistitic tereattmenit alt the boundariies, and $((9.5)),(99.6)$ att the interior poinits. 


\section{DONOR CEELL TYYPE DDIFFERENCE METHOD}

Aa welli known and weny popular ddiffiference method for consenvaltive from fflow its the donor celli diffiference tredhnirque $([[5]],[[7],[[16]]$, [[17] $])$.) IIn ffactt, iitt

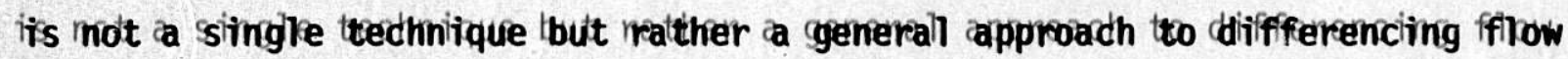
equations. Ssince there are so many warizanits of thiis generall trechniquue, we shalli mott arscribe the dififference method descritbed therein tro any cone source.

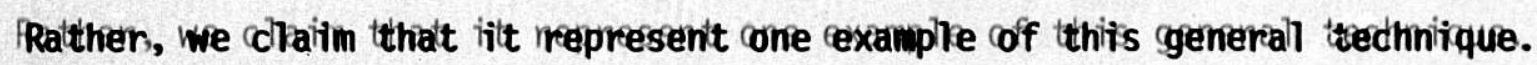

\section{Diffference Equateirons}

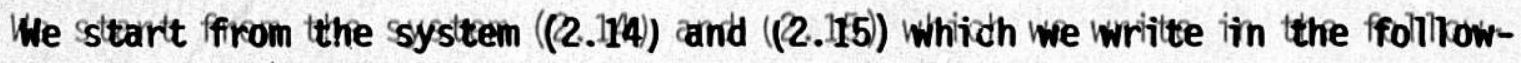
ing form.

$$
\begin{aligned}
& \frac{\partial p}{\partial t}+\frac{\partial}{\partial x}((\partial u)=0 \\
& \frac{\partial G}{\partial t t}+\frac{\partial}{\partial x}((u G))=-\frac{\partial p}{\partial x}-f f G \mid G G / / \partial D p-\rho g
\end{aligned}
$$

Leet the intervall $[[0, L]]$ tbe subibliviided by an cequaliny spaced mesh where

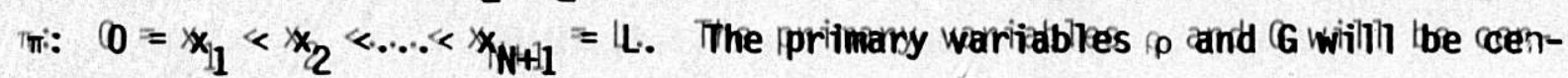
trered diiffiferenitlyy on the mesh. Thatt its,
(a) $\quad p_{i j}^{m} \doteq p\left(\left(x_{i j+x_{2}}, t^{m}\right)\right)$,
b) $\quad\left(G_{i j}^{n} \cong G\left(x_{i j},, t^{n}\right)\right)$,

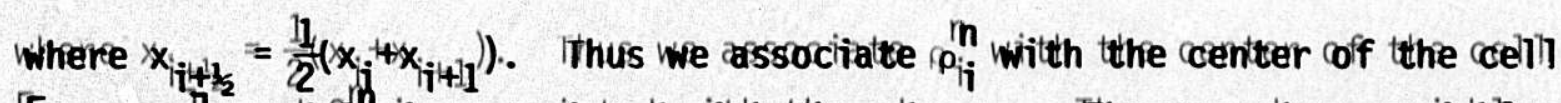

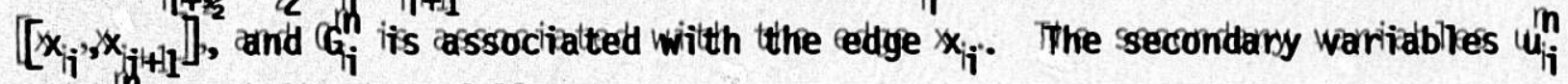

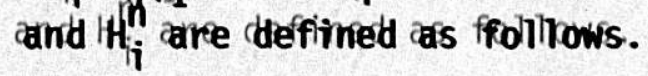

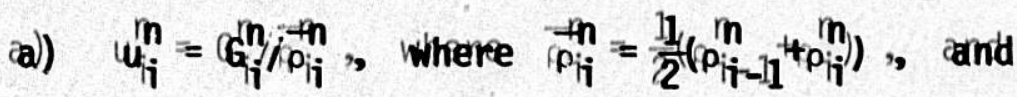
b) $H_{i j}^{m}=a^{2} m_{p_{i j}}^{m} / h_{p_{R}} g-c_{R}$.

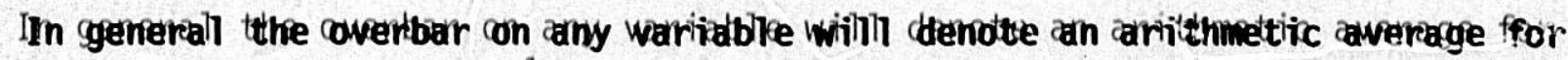

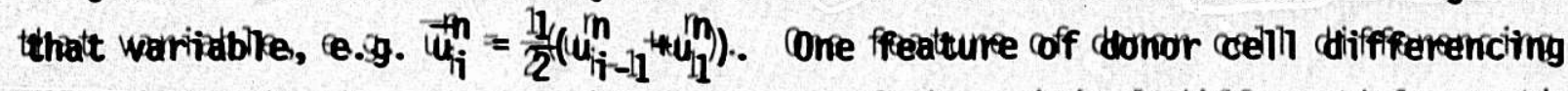

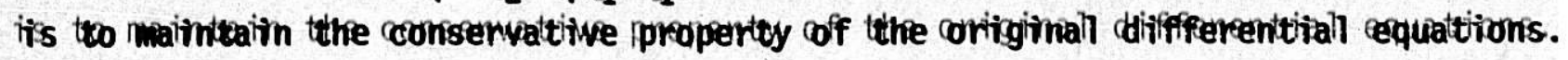


To thits end, the conitinuility and Imomentum equations are ceach initegraited over a

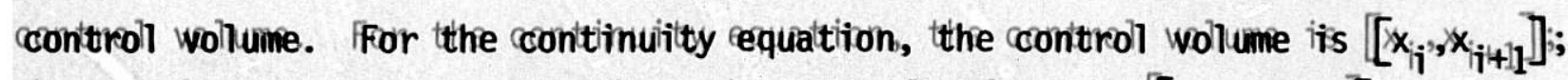
whereas for the momentum equation, the conttroll wollume $i$ is $\left[\left[x_{i j-1 / 2}, x_{i j+\frac{1}{2}}\right]\right.$.

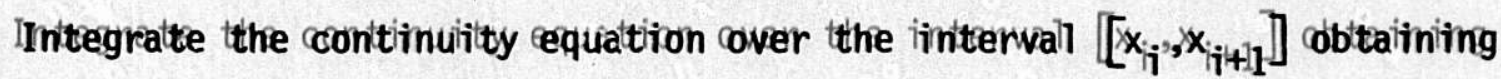

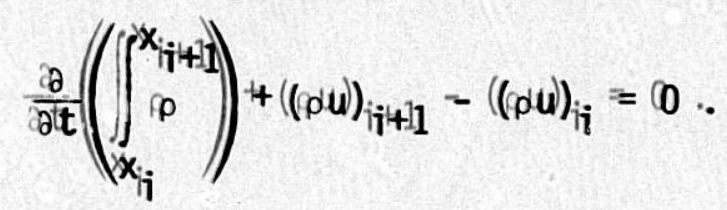

We make the approximaite

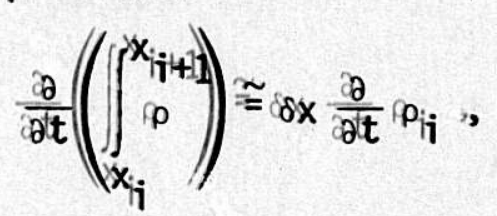

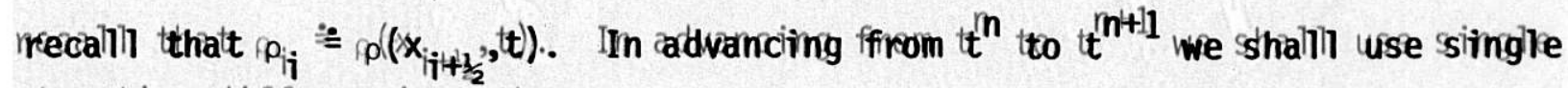
sittep ttime diiffiferenciing; thus:

$$
\frac{\partial}{\partial t} \rho_{i j} \cong\left(\left(\rho_{i j}^{m+11}-p_{i j}^{m}\right) / \delta \delta^{m}\right.
$$

Donor ccell? averaging iis used con the quantititives $\left((\rho \omega)_{i j+11}\right.$ and $\left((\rho u)_{i j}\right.$. Thiis its deffimed ars froliliows: Leet $0 \leq \theta \leq 11$ ibe giviven, and selt

$$
\begin{aligned}
& \left\langle\theta_{i j}=\theta \operatorname{sitign}\left(\left(u_{i j}\right)\right),\right. \text { and } \\
& \langle p\rangle_{i j}=\frac{11}{2}\left[\left(\left(11+\theta_{i j}\right) p_{i j-11}+\left(\left(1--\theta_{i j}\right)\right) p_{i i}\right] .\right.
\end{aligned}
$$

Then we ssett:

$$
\left((p u)_{i j}=\langle p\rangle_{i j} u_{i j} \cdot\right.
$$

When $\theta=11$ thiis iis the ustuali upwint difffiferencing ((cff. [[7]]), and when $\theta=0$

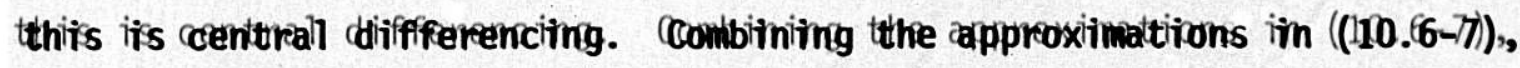

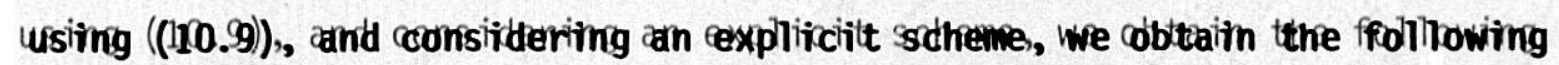

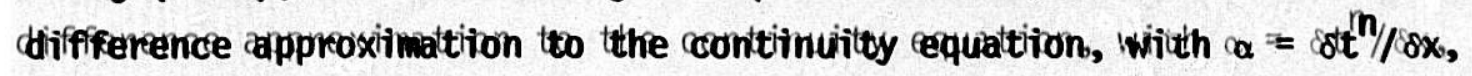

$$
p_{i j}^{m+11}=p_{i j}^{m}-\alpha\left[\left[\langle p\rangle_{i j+11}^{m} u_{i i+11}^{m}-\langle p p>\rangle_{i j}^{m} u_{i i}^{n}\right]\right]
$$

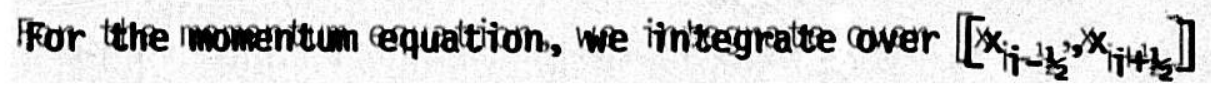




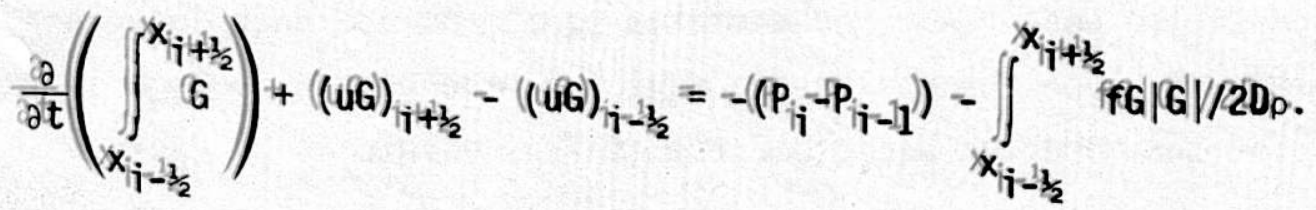

Again we are interested in single time step approximations; thus

$$
\frac{\partial}{\partial t}\left(\int_{x_{i-1 / 2}}^{x_{i++\frac{1}{2}}} G^{2}\right) \cong \frac{\partial x}{\partial t^{n}}\left(\left(G_{i}^{n+1}-G_{i j}^{n}\right)\right. \text {. }
$$

For the moment we are interested in explicicit schemes; thus we will luse the following approximation.

$$
\int_{x_{i-\frac{1}{2}}}^{x_{i+\frac{1}{2}}} f G|G| / 2 D_{\rho} \cong \delta x f G_{i j}^{n}\left|G_{i j}^{n}\right| / 2 D D_{i j}^{-n} .
$$

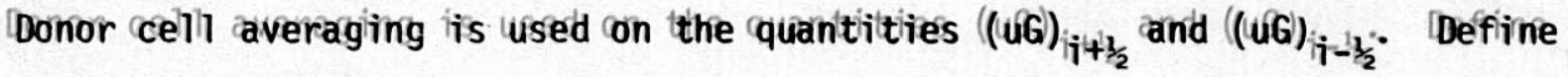

$$
\begin{aligned}
& \text { a) } \theta_{i+1 \frac{1}{2}}=\theta \operatorname{sign}\left[\left(\left(u_{i}^{n}+u_{i+1}^{n}\right) / 2\right]\right. \text {, } \\
& \text { b) }\langle G\rangle_{i+\frac{3}{2}}^{n}=\frac{1}{2}\left[\left(\left(1+\theta_{i+\frac{3}{2}}\right)\right) G_{i j}^{n}+\left(\left(1-\theta_{i+1+\frac{1}{2}}\right)\right) G_{i+1}^{n}\right] .
\end{aligned}
$$

Then we use the approximation

$$
\left((U G)_{i j+\frac{1}{2}} \cong\langle G\rangle_{i j+\frac{1}{2}}^{n} G_{i+\frac{1}{2}}^{n},\right.
$$

where $\vec{u}_{i+\frac{1}{2}}^{n}=\frac{1}{2}\left(u_{i}^{n}+u_{i+1}^{n}\right)$. With these approximations, Eq. ((10.111) takes the foll lowing form.

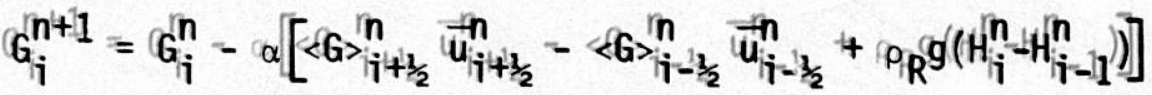

$$
\begin{aligned}
& =\delta t^{n} f G_{i j}^{n} \mid\left[G_{i j}^{n} V / 20 D_{i j}^{-n}\right. \text {. }
\end{aligned}
$$

Equations ( $(10.10)$ and ((10.16) are the basic donor celli difference equations at the interior point. In principle, these equations are conditionalily stable, ij.e. iff $\alpha=\alpha_{n}=\delta t^{n / / \delta x}$ is suitably restricted. We shalli see, however, that for water hammer equations where $|u| \ll$ \&, stabilitity can be achireved conlly when $a$ is severelly restricted. Indeed, it thas been shown ((cf. [[18], [7]] p. 238) that the stabilitity restriction for inviscid flow with thealt conduction in tho 
dimensions given by

$$
\delta t^{n} \leq \frac{(|| u|+| v \mid)) \delta x}{\left((|u|+|v|+a \sqrt{2})^{2}\right.} .
$$

Crearly, when ||$u|| w \mid, \ll a$, this critterion imposes a severe restriction on the time steps.

For this reason we thave imodifited Eq. ((10.116) by Imaking the pressure teem implicicit in time rather than expliicilt. Thus we use $\left(\left(H_{i}^{n+1}-H_{i-1}^{n+1}\right)\right.$ in $((10.116)$ and solve the resulting system lby iiterations on the pressure. Thiis procedure is simillar to that employed by Amsden and Hiv't in the WAQUi code [[19]. The itterattive procedure is

$$
\begin{aligned}
& p_{i j}^{n}((l))=p_{i j}^{n}-\alpha\left[\left[\langle p\rangle=((l--1)) u_{i j+1}^{n}-\langle\hat{p}\rangle_{i j}^{n}\left((l-11) u_{i j}^{n}\right],\right.\right. \\
& \hat{H}_{i j}^{(l e)}=a^{2} \hat{p}((l)) / p_{p_{R}} g-C R \text {, }
\end{aligned}
$$

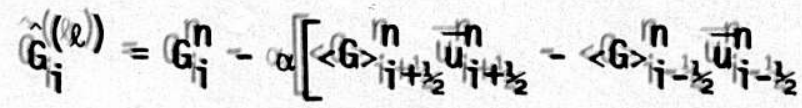

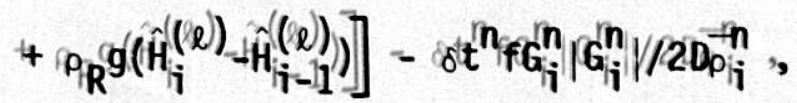

$$
\begin{aligned}
& \hat{u}_{i}^{(l e)}=\hat{G}_{i}^{(l e)} / \overline{\hat{p}_{i j}^{(l e)}}
\end{aligned}
$$

where

$$
\hat{\rho}_{i j}^{((0))}=\rho_{i j}^{n-1}, \quad \hat{u}_{i j}^{(0)}=u_{i j}^{n-1}, \quad \hat{G}_{i j}^{(0)}=G_{i j}^{n-11} .
$$

In this sttudy we used $l=2$ iin these iitterations so that $p_{i}^{n+1}=\rho_{i}^{(2)}$ and $G_{i}^{n+1}=\hat{G}_{i}^{(2)}$. Using cone corrective iitteration in this way ratised the stabilititity coritterion from a wery smalh wallue to a wallue hear the usual couranit condilition. That ivs, het $\left.o_{0}=11 /\left(a^{2}+\left|u^{n}\right|\right)\right)$ where ||$u^{n} \mid=\max \left\{\left|u_{i}^{n}\right|: 11 \leq i \leq \mid N+1\right\}$, then the

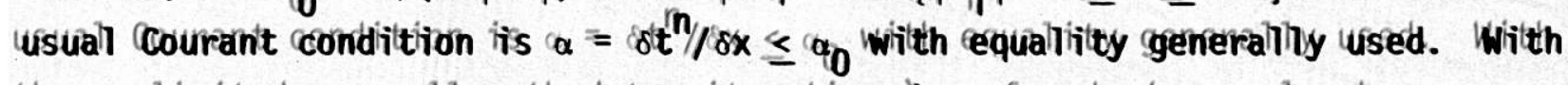
the explicicit donor celll method (no iiteraltions)) we found $\alpha / \alpha_{0}<<1$; whereas with two corrective itterations $\alpha / \alpha_{0}$ i is near one.

\section{Boundary Condititions}

Recall that at the lieft boundary the densiity its specificied by

$$
\left.\rho\left(0, t^{n}\right)\right) \equiv p_{\frac{1}{2}}^{n} \equiv p_{O R} \text {. }
$$


Thus iit remains to detemmine $G_{1}^{n}$ alt the lrefot boundary. Recaln that in deriving the approximation to the mowenitum fequation, ithe ffirsit interval used in the inttegration ons $\left[x_{3 / 2}, x_{5 / 2}\right]$; thus we thave the thalif iontterwal $\left[\left[x_{1}, x_{3 / / 2}\right]\right.$ which thas not tbeen accounited for in this iprocess. We could iderive an equaltion

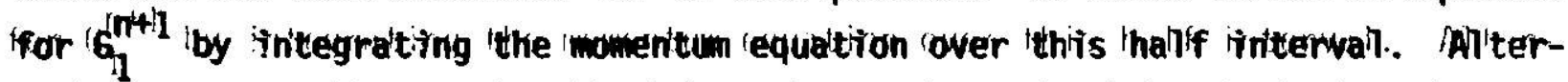

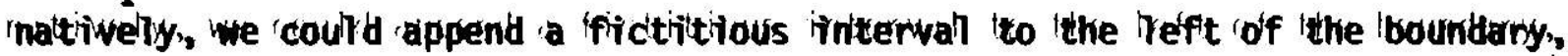
cextrapolate to robtain wahues of the variables ion thiis ifniterval,, and then 'use the bonor cell diffiference technique to dbtain the approprialte equation. This Vattter approach is in the spirritt of idonor cell diffiferencing and we shall use thits approach at the bountaries. To this send, we appent wesh cell $\left[\left[x_{0}, x_{1}\right]\right.$ ] wilth $x_{0}=-\delta x\left(\left(x_{11}=0\right)\right.$ to the coriginal inesh, and use hinear extrapolation to

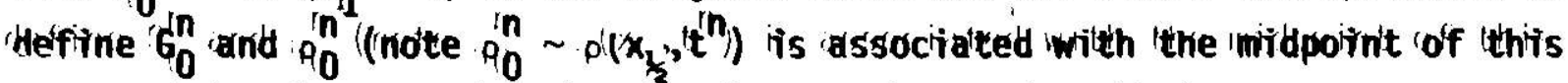
cextra (celli)). Applying IEq. (110.116) for $1 i=1$, , we then firind

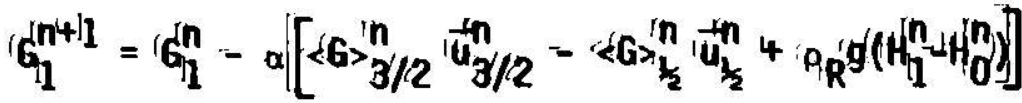

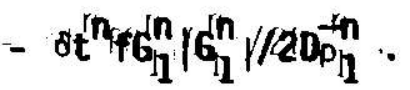

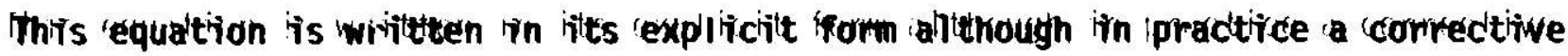
ilteration io (done with this equation just is with the initerifor equations.

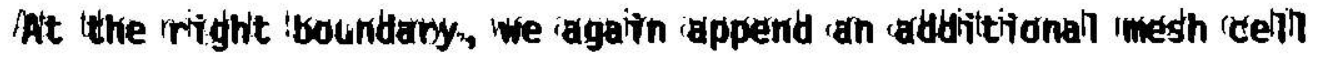

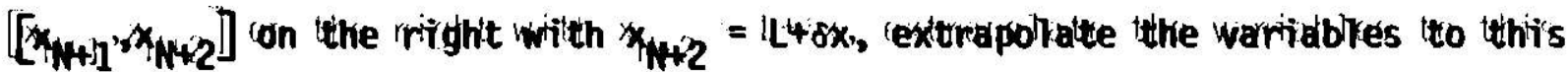

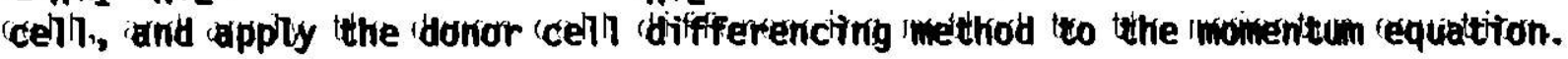
We ffint

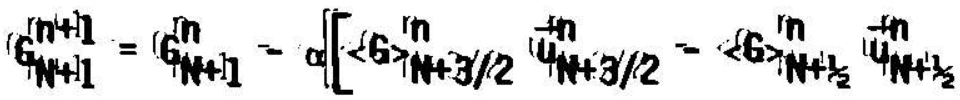

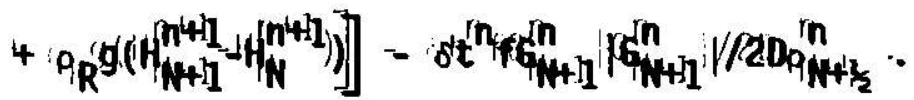

There are several aspedts of thits equation thatt are wrorth motting. Fiirsit, iit

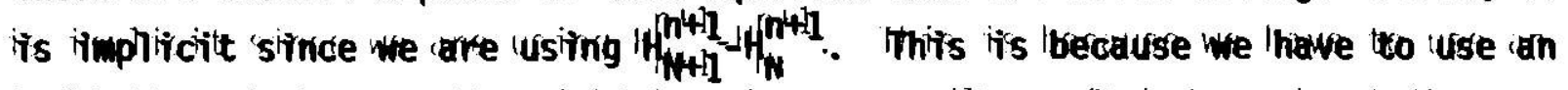

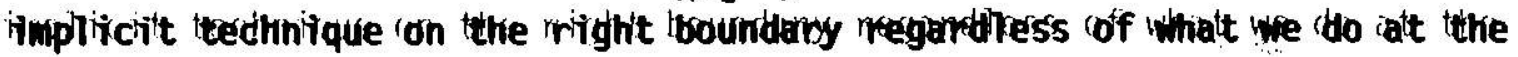

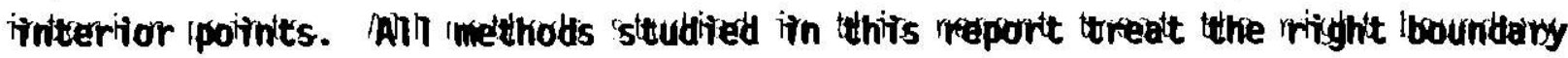

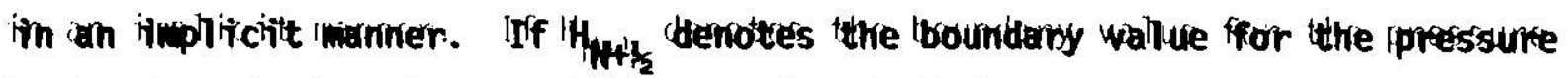

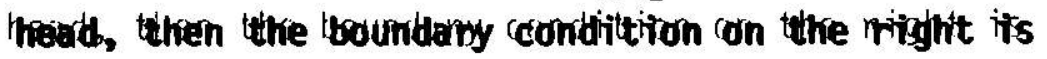




$$
G_{N+1}^{n+1}=u_{0} \tau\left(t(t) P_{N+1 / 2}^{n+1}\left[H_{N+1 / 2}^{n+1} / H_{0}\right]_{0}^{1 / 2}\right.
$$

We use En. ((110.20) iin EEq. ((10.119)), cobsenve that $\left.H_{N+1}^{n+1}-H_{N}^{n+1}=2\left(h_{N+1 / 2}^{n+1}-H_{N}^{n+1}\right)\right)$, and mote that $H_{N+\frac{1}{2}}^{n+1}=a_{P_{N+\frac{1}{2}}}^{n+1 / 1} a_{R} g=C_{R}$. We then wish to solive the equation

$$
\left.f\left(c_{p}\right)\right)=c_{11} p\left[\left[c_{2} p-c_{3}\right]^{\frac{1}{2}}+2 \operatorname{ara} p_{R} p\left(\left(c_{2} p-c_{3}\right)-z=0\right.\right.
$$

Where

$$
\begin{aligned}
& \text { a) } c_{11}=u_{0} t^{t(t)} \text {, } \\
& \text { (b) } c_{22}=a^{2} / p_{p_{R}} g H_{0} \text {, } \\
& \text { c) } c_{3}=c_{R} / / H_{0} \text {, }
\end{aligned}
$$

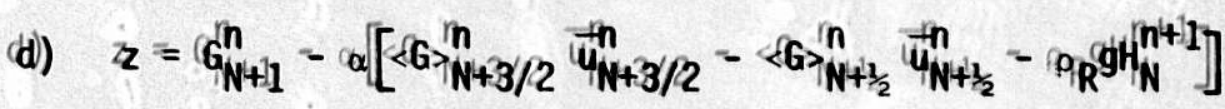

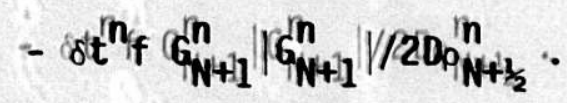

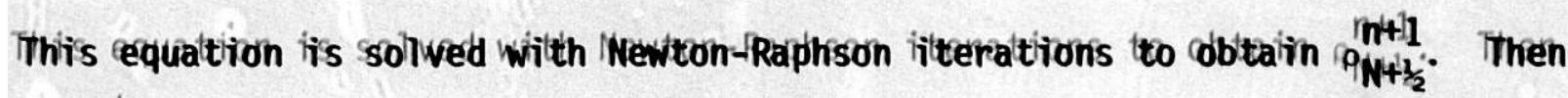
we set

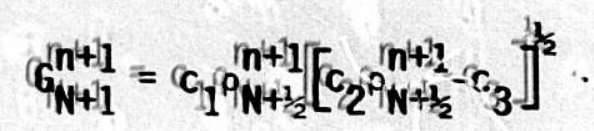

to complete the calloulation alt the rifishit boundany. 
11. CCOAPARISON OF THE METHOD OF CHAPRACTERISTICS, THO-STEP LLAX-WENDROFF, AND DONOR CELL TYPE DIFFERENCING

In this section we compare approximations to the rigid wall rnodel with the conservative governing Eqs. ((8.11). In the case of the ritgitt walli model governed by the system ( $(2.14)$ and $((2.15))$, the primany dependent variables are $((0,(G))$; whereas in the elasticic wall model, the primany variables were $((u, H)$. For purposes of comparison with the elrastic wall nodel (we may mote here the comparison is valitid onlly iff $p=q_{R}$ and the acoustic speed has the same wallue in tboth rmodels)),

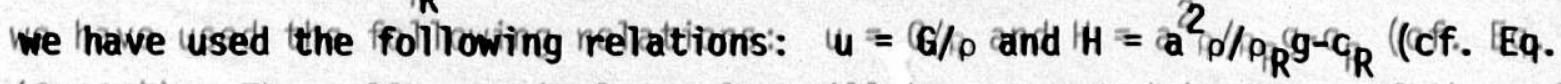

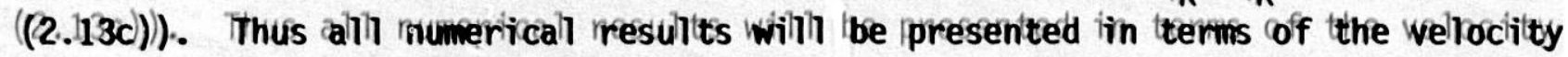
w and the pressure thead $1 \mathrm{H}$. In this section we have restricted cour alttention to the case of $\mathrm{Im}=1 \mathrm{l}$. Moreover, when presenting mumericall results, cour altitention will be focused as before at the toime $t=77.7728$ and on the welrocilty alt this tiime.

Benchmark Walues

For thiis studidy the benchmark wallues are dettenwined by the Imethod cof characterisistics with $N=80$ and hinear interpollation. To hiighliight the diffiferences

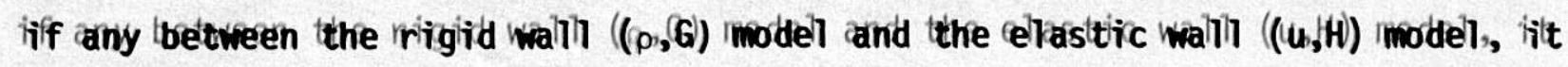
is worth comparing the benchmark wallues fror the models. To thiis end, Tablie lil. ll presenits the welrocilties alt $t=7.7728$ and alt selected poinits $\overline{\bar{x}}=0,0.2,0.4,0.6$, and 0.8 allong the pipe.

TABLE l11.1 Compariison of BBendmark Wallues

\begin{tabular}{|c|c|c|c|}
\hline $\bar{x}=x / L$ & $\begin{array}{c}((0, G) \text { moddel } \\
\text { Linnear } \\
\text { Interpollation } \\
W=80\end{array}$ & $\begin{array}{c}\left(\left(u_{0}, H\right)\right) \text { moddel } \\
\text { Limear } \\
\text { Interpoliattion } \\
N=80\end{array}$ & 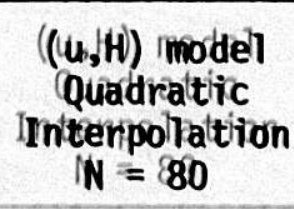 \\
\hline 0.0 & -0.291567 & $=0.2911590$ & $=0.2991594$ \\
\hline 0.2 & -0.2901154 & $=0.2901776$ & $=0.290428$ \\
\hline 0.4 & -0.268600 & $=-0.2686117$ & $=0.268613$ \\
\hline 0.6 & $=0.2499244$ & -0.249254 & $=0.249251$ \\
\hline 0.8 & $=-0.11613666$ & $=0.116133711$ & $=0.1161368$ \\
\hline
\end{tabular}


From this table we conclude that the mumerical differences between these wo model's are negligible for the purposes of this study. Thus the numericall resulits presented in section 77 can be directlly compared with the numericall result in this section. However, in this section we have selected the $((0,9)$ model with hivnear initerpolation and $\mathrm{N}=80$, as the benchmark, for the sake of consisistency.

\section{Domor celli Diffiferencing I Method}

The donor celly tedhnique which we thave considered consisists of tho versions - an expliicilt wersion and the version with two itterative corrections which we willh refer to as the quasii iiterative wersion. Each wersion has three parameters associalted with iit. The ffirst iis the mumber of mesh cellis in which is aliso the number of wariables associatted with each dependent variable. The second parameter is $\theta$ which deterwines the donor cell averaging as discussed in section 110 . ((Recall) that $\theta=0$ corresponds to centrall differencining whereas $\theta=11$ corresponds to lupwind differencing.)) The thiird parameter is the time sttep ssize which we measure in terms of the ccourant steep. That iis, we set $\alpha_{0}=1 \mathrm{l} /\left(\left(\mathrm{a}+\left|\vec{u}^{\mathrm{n}}\right|\right)\right), \alpha=\delta \mathrm{t}^{\mathrm{n}} / / \delta \mathrm{x}$ and use the ratio $\alpha / \alpha_{0}$ as a measure of the sitep ssize. Thus $\alpha / \alpha_{0}=1$ is ttaken as the morm for an expliicilt method. Before comparing the donor celll technique with other methods we shalll sellect cone versition and for this wersion we shalli select alppropriate vallues for $\theta$ and $\alpha / 0 ; 0$.

We fiirsit compare the expliicilt wersion and the quasi-iitterative version with respect to the stabiliitty livimitt. Thalt is, both wersions are condiltionaliny stable in the sense that for $\alpha / \alpha_{0}$ suffificientily smalil, the rmethod is strable. We deterwine whether a method is stable cor not by cexamining the welocitity proffilie mear the end of the transizent alt $t=77.728$. IIf the method is sstable, thiis profilite is nonotoriic.

For the expliicilt wersition of the donor cell teechnique we sett $\theta=11$ ((upwind diffiferencing) ssince we would cexpect this wallue of $\theta$ to lprovide the

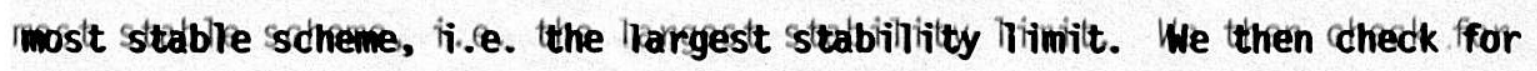
stabililitty with warious wallues for the rattio $\alpha / q_{0}$ with $N=5$ and 110 . The resultts are presented in Trable 111.2. IIn thiis ttable we thave allsso shown the

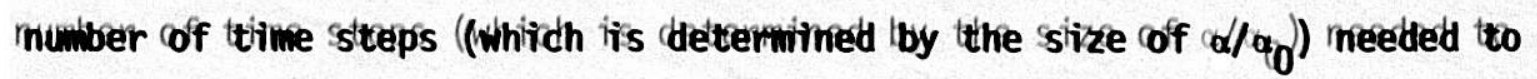
reach $t=77.7288$. 
TABLE li1.2 Stabiliitey Limiits for Expliicit Donor (Celli Method, $\theta=11$

\begin{tabular}{|c|c|c|c|c|}
\hline \multirow[b]{2}{*}{$\alpha / \alpha_{0}$} & \multicolumn{2}{|c|}{$N=5$} & \multicolumn{2}{|c|}{$N=110$} \\
\hline & Stable & $\begin{array}{l}\text { \# Time } \\
\text { Steeps }\end{array}$ & Stable & $\begin{array}{l}\text { \# Time } \\
\text { Steeps }\end{array}$ \\
\hline 11.0 & No & 47 & No & 83 \\
\hline (0.1 & No & 371 & iNo & 7728 \\
\hline 0.05 & res & 735 & No & 11451 \\
\hline 0.025 & Yes & $\cdots$ & Wes & 2890 \\
\hline
\end{tabular}

From this table iit is cllear that the explicicit wersion is mot practicall for solving waterhamer problems of the type consiidered in this sttudy. In Table ll1.3, we present stabilitity resullts for the quasii-iiteraltive version of the donor celli method. The resulits in this table are the same for $\theta=0.0$ cor 11.0.

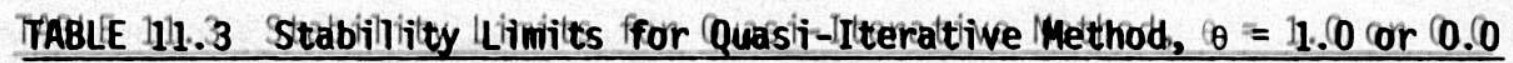

\begin{tabular}{|c|c|c|c|c|c|c|c|c|}
\hline \multirow[b]{2}{*}{$\alpha / 0,0$} & \multicolumn{2}{|c|}{$N=5$} & \multicolumn{2}{|c|}{$N=110$} & \multicolumn{2}{|c|}{$N=20$} & \multicolumn{2}{|c|}{$N=40$} \\
\hline & Stable & $\begin{array}{l}\text { A Time } \\
\text { steeps }\end{array}$ & Stable & $\begin{array}{l}7 \text { Time } \\
\text { steeps }\end{array}$ & Stable & $\begin{array}{l}\text { Thime } \\
\text { Streps }\end{array}$ & Stablipe & $\begin{array}{l}\text { A Time } \\
\text { Streeps }\end{array}$ \\
\hline 11.0 & No & 47 & No & 83 & No & 1155 & $1 N 0$ & 299 \\
\hline 0.9 & No & 50 & No & 96 & Yres & 1164 & No & 326 \\
\hline 0.8 & No & 54 & res & 1101 & Yes & $\cdots$ & Yres & 3771 \\
\hline 0.7 & Yes & 56 & Yes & $\cdots$ & Wes & $\cdots$ & Wess & $\cdots$ \\
\hline
\end{tabular}

Trable ll1. 3 shows that the quasii-iitterattive wersion of the donor celli

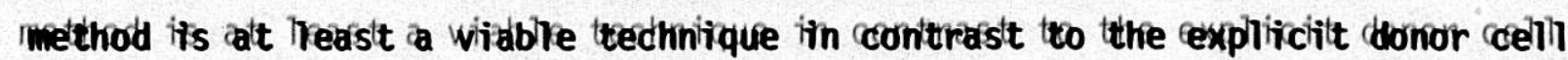
methrot.

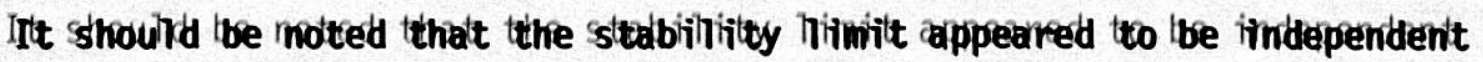
of the mumber of conrective iitterattions. Fror thiis reason we sselectred two 
itteraltions ffor this s'tudy. From this point on we sha?l consider onlly the quasi-iilteraltive wersion.

We now consitder the selection of the donor cell aiveraging parameters. To

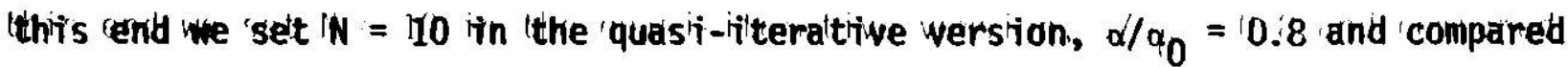
the wellocilties alt $1 t=7.728$ for $\theta=0.0,10.5$, and 11.10 . The resuitts are shown in Table 111.4.

TABLLE lil. 4 Comparison of /Accuracy in Welocilty alt it $=7.728$ and Spattial Points $\bar{x}$ for $\theta=0.0,0.5,1.0$

\begin{tabular}{|c|c|c|c|c|c|c|}
\hline \multirow{2}{*}{$\bar{x}=x / A L$} & \multicolumn{2}{|c|}{$\theta=0.0$} & \multicolumn{2}{|c|}{$\theta=0.5$} & \multicolumn{2}{|c|}{$\theta=1.0$} \\
\hline & wellocility & $\begin{array}{c}\% \\
\text { r.e.e. }\end{array}$ & velocilty & $\begin{array}{c}\% \\
\text { r.te. }\end{array}$ & welocity & $\begin{array}{c}\% \\
\text { r.e.e. }\end{array}$ \\
\hline 10.0 & -0.296360 & 1.64 & -0.296336 & 11.64 & -0.296312 & 1.63 \\
\hline 10.2 & -0.2911229 & 10.37 & -0.2911227 & 0.37 & -0.2911224 & 0.37 \\
\hline 10.4 & -0.255267 & 4.96 & -0.255281 & 4.96 & -0.255294 & 4.95 \\
\hline 0.6 & $=0.2675553$ & 7.35 & -0.267489 & 7.32 & -0.267426 & 7.30 \\
\hline 10.8 & -0.1163697 & $1 . .45$ & -0.1163648 & 11.41 & -0.1163670 & 1.43 \\
\hline
\end{tabular}

IFrom lthis Itable we conchulde that there ios ino cessential diffference in the

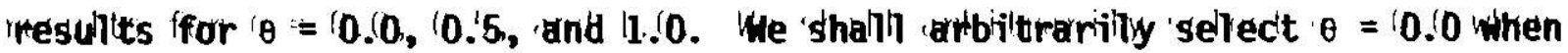
compariing wilth the cother inethods.

In sumany., we thave selyedted the (quasi-iiterattive wersion (ttwo corrective iitserattionis) with $\theta=\left(0.0\right.$ and $\alpha / \alpha_{0}=(0.8$ as the represenitative (donor cell wethod for comparison with the cother 'methods.

Comparitson off the Three Methods

We mow coompare the accuracy of the three methods under consitderation, that

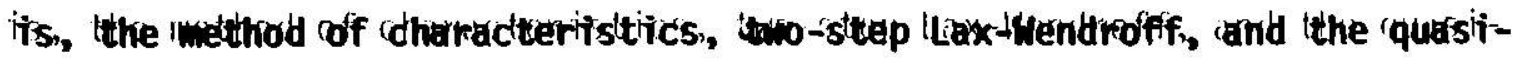

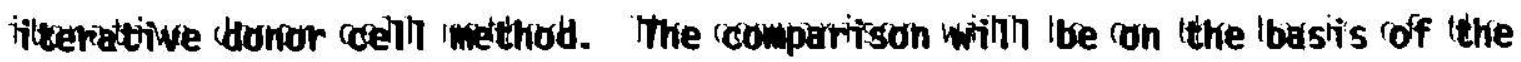

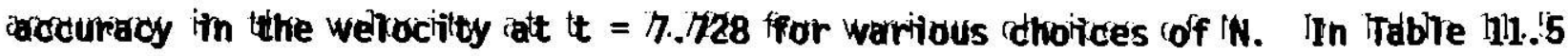

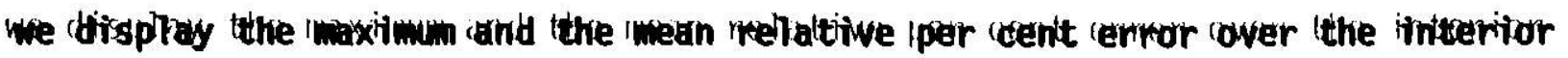
prointes (IInt.) $\bar{x}=10.2,0.4,(0.6$, and $(0.8$. In caddiition, we also (tisplay the 
TABLE 111.5 Comparison of Errors in the Velocity at $t=77.7228$

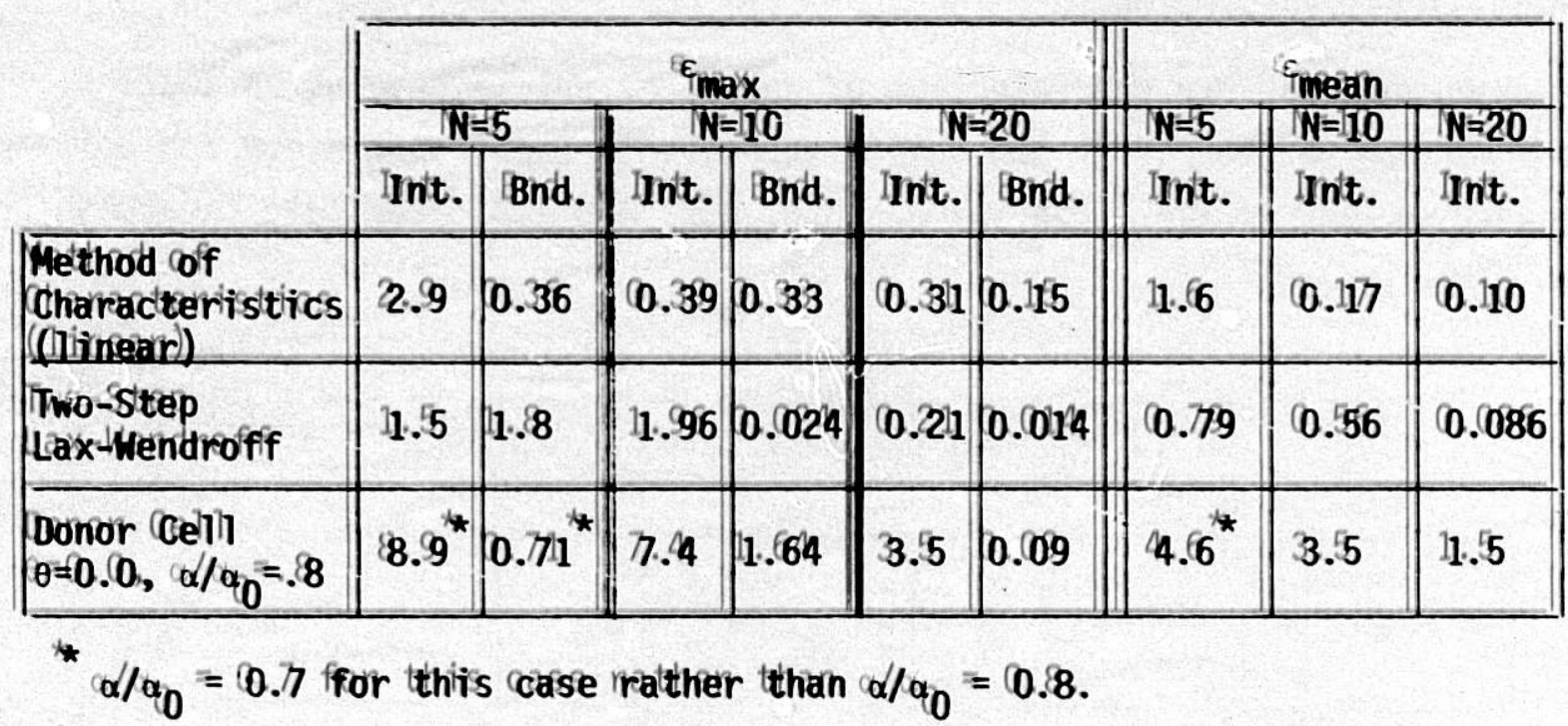

rellative per cent error alt the reservoir lboundary ((Bnd.)). The resulits in this

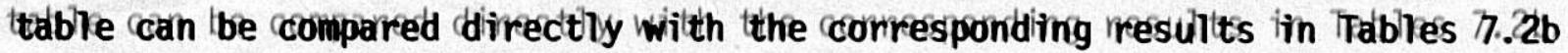
and $7.3=7 . .5$.

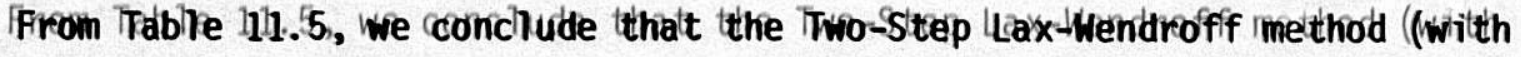
method of characterisistics used alt the lboundaries) /has comparable accuracy witth the Imethod of characterisistics. The donor celli method is cllearliny mot of comparable accuracy. The proper treatment cof tboundany condititions its coritticall in cobtaining accuralte resultts. Foor example, in the two-sttep Lax-Wendrofif method (which is ssecond corder accuratte in tiime alt the initeriion poinits)), we

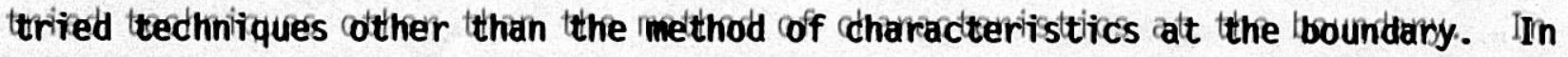

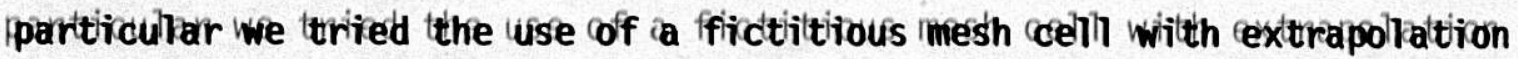
ssimillar to that which was lused in the donor celll imetthod. IIn teemis of accuracy,

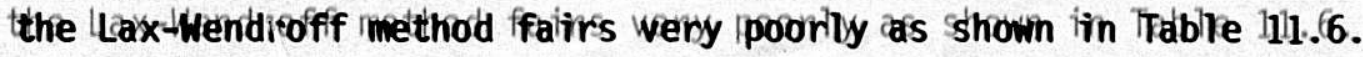

TABLEE 111. 6 Comparison of Treatement of Boundany Condititions with Two-steep Lax-Wendroffif het thod

\begin{tabular}{|c|c|c|c|c|c|c|c|c|c|}
\hline \multirow{3}{*}{$\begin{array}{l}\text { Boundaryy } \\
\text { Treattwent' }\end{array}$} & \multicolumn{6}{|c|}{$\operatorname{lnmax}$} & \multicolumn{3}{|c|}{ Gmean } \\
\hline & & 5 & & 10 & & 20 & $M=5$ & $N=10$ & $N=20$ \\
\hline & IIn't. & Bind. & Intt. & EBnid. & Innt. & Bnid. & Intit. & Intt. & In't. \\
\hline 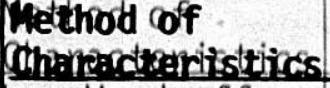 & 11.5 & 11.8 & 11.996 & (0.224 & 0.221 & (0.0114 & 0.779 & 0.56 & 0.08 \\
\hline Laxk-Wentrivo & 32.0 & 15.0 & 124.11 & 11.6 & 77.04 & 11.3 & 118.2 & 6.0 & 2.4 \\
\hline
\end{tabular}


We thave always ltried to use the llargest time steps possible, subjedt to the stabibihilty criiterion imposed for any iparticular Imethod. IIt iniight ibe supposed thalt addititionall accuracy could be 'gainned by reducing the time sitep tbelow the 'stabilliity himilt. However, Inosit 'of the Imethods discussed in this stubly thave rnumerical diffffusion associalted witth them whidh increases as the ttime step is reduced. We iinhusitralte thiis siltuation with the donor cell Inethod for $1 N=5,0, \theta=0.0$ and $\alpha / \alpha_{0}=0.25,0.5$, and 0.7 where 10.7 is inear the

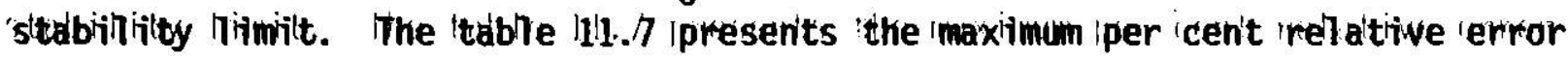
in the welrocilty alt $i t=7.728$ for these three ccases.

TAABLE l1l..7 Comparison off Accuracy for Three Time Step Sizzes

\begin{tabular}{|c|c|c|}
\hline$\alpha / q_{0}$ & $\varepsilon_{\max }$ & $\begin{array}{c}\text { ITime } \\
\text { Steps }\end{array}$ \\
\hline 10.7 & 8.9 & 56 \\
0.5 & 116.9 & 83 \\
0.25 & 20.6 & 155 \\
\hline
\end{tabular}


12. Conclusirons

In thiis study, we thave compared the accuracy obtained with seven dififfercenit approximations to a siingle piipe watter thammer probliem. In the frollinowing trable we summarize the resultes for the accuracy of the sseven mithoods. We thave selkectred as tbefore $t=77.7228$ and give the maximum rellative per cent cenror in the welocitity att the reservoir and the frour interitor poinits $\bar{x}=0.2,0.4,0.6$, and 0.8 . From these resultts presented in Trable 111.8 , iit its cllear that friom the poinit of witew of accuracy, Two-Steep Lax-Whendroffif and the method of charac-

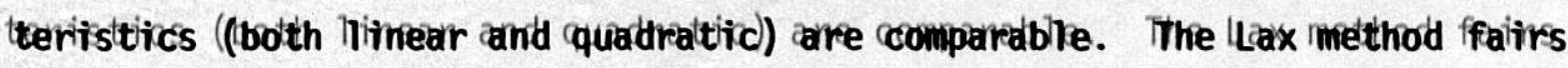

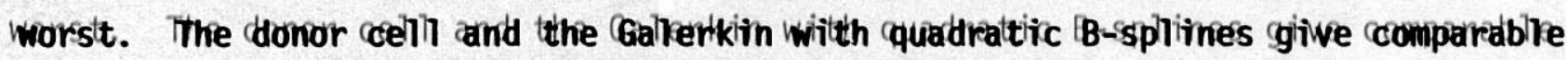
accuracy, tout the method of characteriistivics and two-steep Laax-Wendroffif methodis cllearly sstand cout, that iis, produce the besit accurarcy.

TAABLE ll11.8 Compariison of AAccurarcy in the Wellocitoy alt $t=77.7728$

\begin{tabular}{|c|c|c|c|}
\hline & $N=5$ & $N=110$ & $N=20$ \\
\hline M.0.C. ((quard)) & 11.995 & 2.11 & 0.118 \\
\hline Two-Steep Lau-Wentroofff & 11.8 & 11.996 & 0.211 \\
\hline M.O.C. (Limear)) & 2.99 & 0.36 & 0.31 \\
\hline Dornor (cellit $\left(\left(\theta=0.0, \alpha / / \phi_{0}=.8\right)\right.$ & 8.9 & 77.44 & 3.5 \\
\hline Galkerkin ( $(k=3, w=11))$ & 1111.3 & 2.6 & 11.11 \\
\hline Galkerkin $((k=2, v=11))$ & 23.8 & 5.4 & 11.99 \\
\hline LLax & 50.9 & 174.9 & 4.9 \\
\hline
\end{tabular}

\section{ACOWOWLEDGWENT}

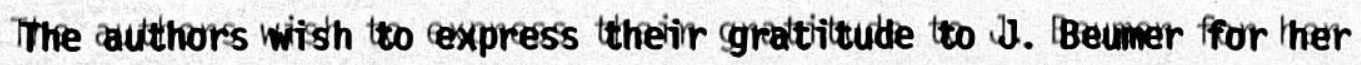

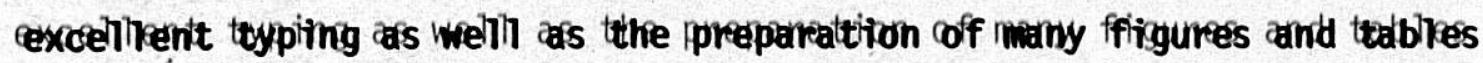
cappearing in thiis reaport. 


\section{REFERENCES}

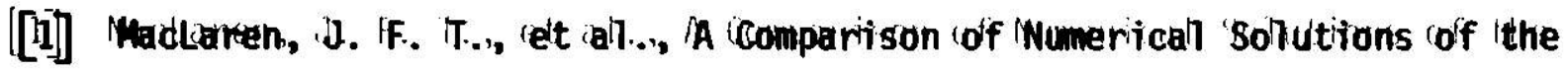
Unisteady IFlow IEquations applired Ito IReciprocationg (Compressor Systems, W. Medh. IEngng. Scij. 1975, 177, 1No. 5., 1pp. 2/17-279.

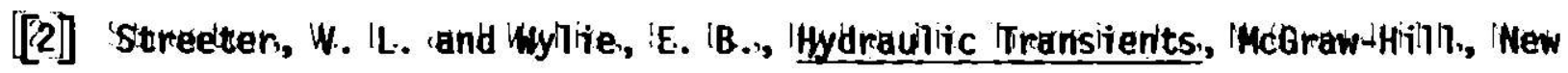
ronk, 11967.

[[8] Courant, IR. and IFriebriidks, IK. (0.,, Supersonic ffllow and Shodk Waves, Intiterscience |Publ.., New Mork., l1948.

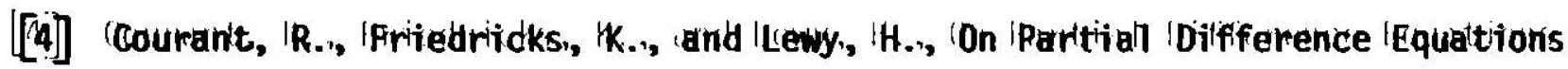
of Malthemalticall IPhysics, I1BM Journall III, Mar 11967, Ipp. 2115-234 ((Eng]iish

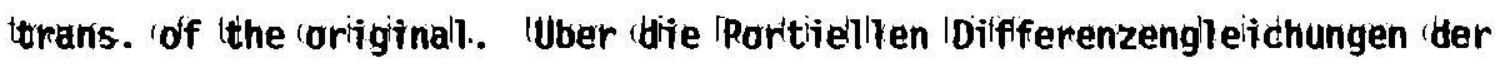

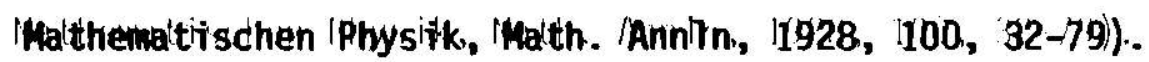

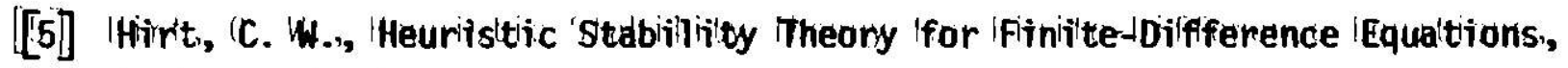
U. Comp. IPhysics, i2, 1968, ipp. 339-355.

[[6]] LLax, IP. ID., Weak Sollutions iff INonlitinear /Hyperbolliic IEqualtions and Their

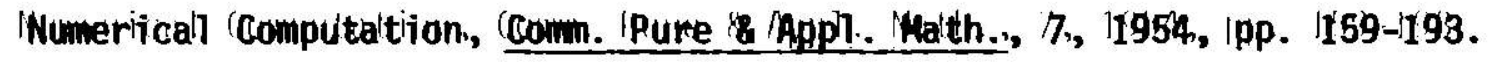

[[7] IRoadhe, IP. U.., Computaltional IFlluitd IDynaniics, IHermosa IPubl.., New Mexico, 1976 .

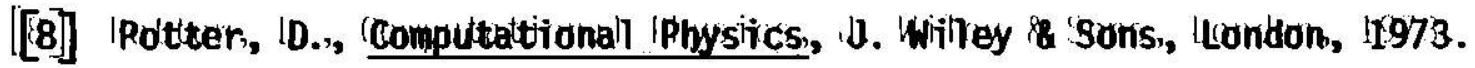

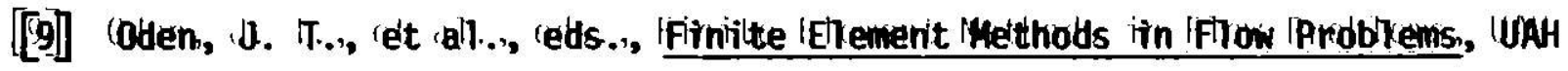

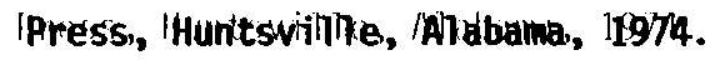

[[10]] Leaff, (6., celt all.., IDISPL: /A soffitware IPadkage ffor (One and Two Spattialliny Limensioned Kionetiocs-Diffifusion IProblemens, Argonne Naltionali LLaborattony

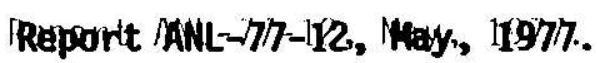

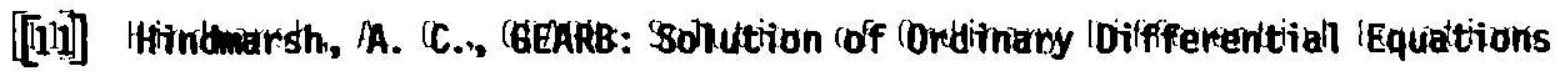

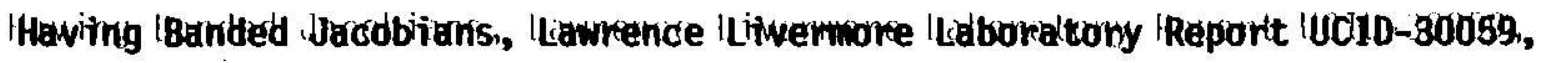
Hev. 1, Marech 19975 .

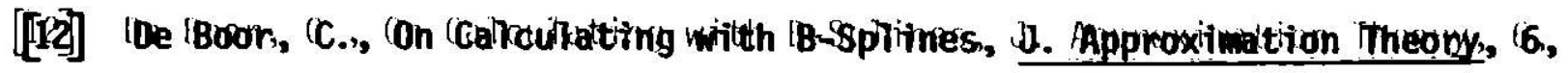
15972, Ipp. $150-62$. 


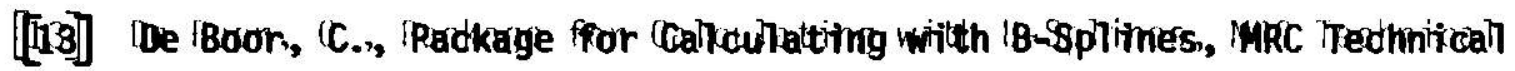

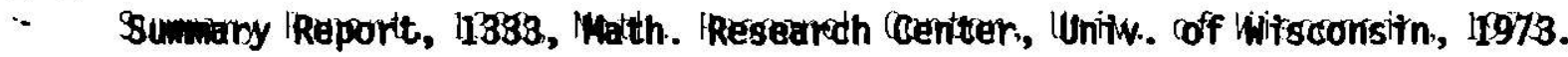

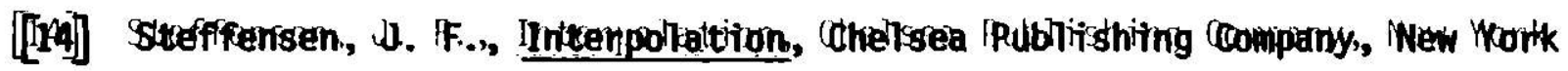
((119960).

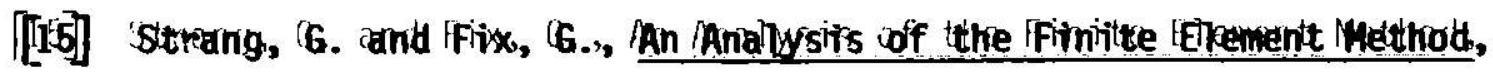

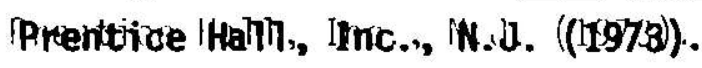

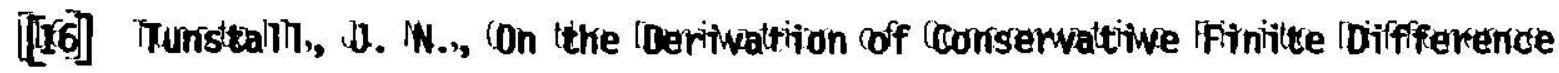

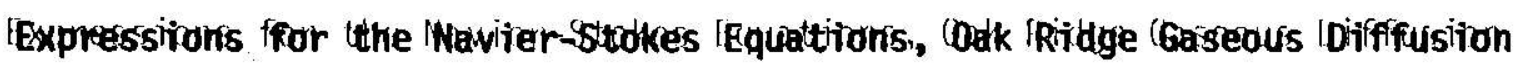

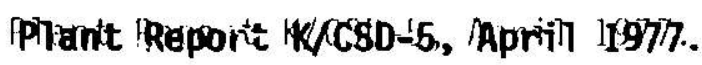

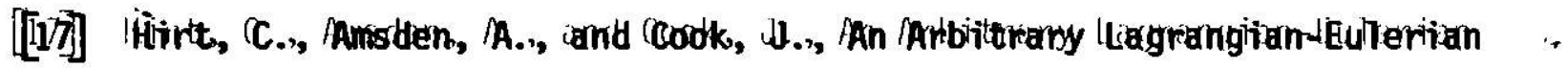

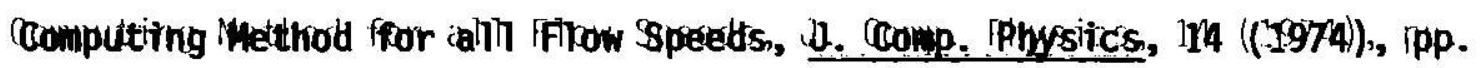
$227-253$. 